\title{
Some new invariants of vector bundles on smooth projective surfaces
}

\author{
Igor Reider
}

\begin{abstract}
Let $\mathcal{E}$ be a rank-2 bundle over a smooth complex projective surface $X$. Whenever the subscheme of zeros of a global section of $\mathcal{E}$ is zero-dimensional, it gives a geometric realization of the second Chern class $c_{2}(\mathcal{E})$. Taking the incidence correspondence

$$
\mathcal{Z}=\left\{(x,[e]) \in X \times \mathbf{P}\left(H^{0}(\mathcal{E})\right) \mid e(x)=0\right\} \stackrel{p_{2}}{\longrightarrow} \mathbf{P}\left(H^{0}(\mathcal{E})\right)
$$

and considering the Zariski open subset $U_{f} \subset \mathbf{P}\left(H^{0}(\mathcal{E})\right)$ over which the morphism $p_{2}$ is finite, we have

$$
\mathcal{Z}_{f}=p_{2}^{-1}\left(U_{f}\right) \longrightarrow U_{f}
$$

a family of zero-dimensional subschemes of $X$ of length $\operatorname{deg}\left(c_{2}(\mathcal{E})\right)$. This can be viewed as a distinguished geometric representative of $c_{2}(\mathcal{E})$.

We define a new invariant of $\mathcal{E}$ which can be viewed as a 'lifting' of $c_{2}(\mathcal{E})$ to $U_{f}$. This invariant is a sequence of sections of some coherent sheaves on $U_{f}$. These sheaves are built from the following cohomology cup-products:

$$
\begin{aligned}
\gamma_{1} & : H^{1}\left(\mathcal{E}^{*}\right) \otimes H^{0}(\mathcal{E}) \longrightarrow H^{1}\left(\mathcal{O}_{X}\right), \\
\gamma_{2} & : S^{2} H^{1}\left(\mathcal{E}^{*}\right) \longrightarrow H^{2}\left(\mathcal{O}_{X}\left(\operatorname{det}\left(\mathcal{E}^{*}\right)\right)\right)
\end{aligned}
$$

The main property of our invariant is as follows: either it determines the family $\mathcal{Z}_{f} \stackrel{p_{2}}{\longrightarrow} U_{f}$, or the vector bundle $\mathcal{E}$ is 'special'. The speciality is expressed in terms of the special geometry of zero-loci of global sections of $\mathcal{E}$ and the special geometry of $X$.

The sequence of sections entering the definition of our invariant is obtained by starting with the one defined by the cup-product $\gamma_{2}$ and deriving others inductively by using a geometric interpretation of a part of the cup-product $\gamma_{1}$ together with the Grothendieck residue map. So one can view our invariant as $\gamma_{2}$ together with some kind of higher-order cohomology cup-products.

The emergence of these higher-order cohomology cup-products is explained conceptually as higher-order derivatives of a natural deformation of $\gamma_{2}$ associated to a certain 'natural' deformation of the complex structure on $\mathcal{E}$. The variety of these natural deformations of $\mathcal{E}$ has all the features of the classical Jacobian of curves: it carries a distinguished divisor which either determines the family $\mathcal{Z}_{f} \stackrel{p_{2}}{\longrightarrow} U_{f}$ or 'sees' that $\mathcal{E}$ is special in the aforementioned sense. An essentially new feature of this Jacobian of $\mathcal{E}$ is that it also carries a variation of Hodge-like structures which arises naturally from our invariant.
\end{abstract}

Received 20 June 2003, accepted in final form 17 February 2004, published online 10 February 2005. 2000 Mathematics Subject Classification 14J, 14J60.

Keywords: vector bundles, surfaces, zero-cycles.

This journal is (C) Foundation Compositio Mathematica 2005. 


\section{REIDER}

\section{Introduction}

Let $\mathcal{E}$ be a rank-2 bundle over a smooth complex projective surface $X$. The basic invariants associated to $\mathcal{E}$ are its Chern classes (viewed as elements of the Chow ring of $X): c_{i}(\mathcal{E}) \in A^{i}(X)$, for $i=1,2$. It is well known that, if $e$ is a global section of $\mathcal{E}$ whose scheme of zeros $Z_{e}=(e=0)$ is zero-dimensional, then the rational equivalence class of $Z_{e}$ is $c_{2}(\mathcal{E})$. In other words $Z_{e}$ is a geometric realization of $c_{2}(\mathcal{E})$. More generally, consider the incidence correspondence

$$
\mathcal{Z}=\left\{(x,[e]) \in X \times \mathbf{P}\left(H^{0}(\mathcal{E})\right) \mid e(x)=0\right\} \stackrel{p_{2}}{\longrightarrow} \mathbf{P}\left(H^{0}(\mathcal{E})\right),
$$

where $p_{i}$, for $i=1,2$, is the projection of $X \times \mathbf{P}\left(H^{0}(\mathcal{E})\right)$ on the $i$ th factor. Taking the Zariski open subset $U_{f} \subset \mathbf{P}\left(H^{0}(\mathcal{E})\right)$ over which the morphism in (0.1) is finite, we obtain the incidence cycle

$$
\mathcal{Z}_{f}=p_{2}^{-1}\left(U_{f}\right) \cap \mathcal{Z} \stackrel{p_{2}}{\longrightarrow} U_{f} .
$$

This family of zero-dimensional subschemes of $X$ of length $\operatorname{deg}\left(c_{2}(\mathcal{E})\right)$ can be viewed as a distinguished geometric representative of $c_{2}(\mathcal{E})$, or what we will call the geometric second Chern class of $\mathcal{E}$. A new invariant of $\mathcal{E}$ proposed in this paper can be considered as a 'lifting' of $c_{2}(\mathcal{E})$ from $A^{2}(X)$ to $U_{f}$. More precisely, our invariant is a sequence of sections of some coherent sheaves on $U_{f}$. These sheaves are built from the cohomological data related to $\mathcal{E}$, hence the name: the cohomological invariant of $\mathcal{E}$.

The second Chern class of $\mathcal{E}$ contains essentially topological information about (0.2): the degree of the morphism. The cohomological invariant of $\mathcal{E}$ is devised to capture the geometry of $(0.2)$. We prove the following property.

Proposition (Proposition 2.3). The cohomological invariant of $\mathcal{E}$ either determines the geometric second Chern class of $\mathcal{E}$ (and the morphism $p_{2}: \mathcal{Z}_{f} \longrightarrow U_{f}$ ), or $p_{2}$ admits a nontrivial decomposition

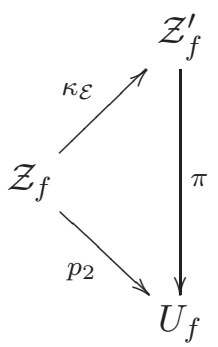

where the morphisms $\kappa_{\mathcal{E}}$ and $\pi$ are finite. Furthermore, the cohomological invariant determines $\mathcal{Z}_{f}^{\prime}$ (and the morphism $\pi: \mathcal{Z}_{f}^{\prime} \longrightarrow U_{f}$ ) unless the fibres of the family $\mathcal{Z}_{f} \stackrel{\kappa_{\mathcal{E}}}{\longrightarrow} \mathcal{Z}_{f}^{\prime}$ are geometric representatives of the second Chern class of some rank-2 bundles over $X$ whose first Chern class is $c_{1}(\mathcal{E})$, i.e. $\mathcal{Z}_{f}^{\prime}$ is a variety parametrizing a family of vector bundles of rank 2 over $X$ and for every $z^{\prime} \in \mathcal{Z}_{f}^{\prime}$ the corresponding vector bundle $\mathcal{E}_{z^{\prime}}$ has the first Chern class $c_{1}\left(\mathcal{E}_{z^{\prime}}\right)=c_{1}(\mathcal{E})$ and its second Chern class $c_{2}\left(\mathcal{E}_{z^{\prime}}\right)$ is represented by $p_{1 *}\left(\kappa_{\mathcal{E}}^{*}\left(z^{\prime}\right)\right)$.

The decomposition (0.3) can be viewed as some kind of speciality of $\mathcal{E}$, and the degree of $\kappa_{\mathcal{E}}$ is a numerical measure of this speciality. This will be called the degree of the cohomological invariant of $\mathcal{E}$. In order to see what bearing this notion of speciality has on the properties of $\mathcal{E}$ (and on the geometry of $X$ ), consider the evaluation morphism

$$
H^{0}(\mathcal{E}) \otimes \mathcal{O}_{X} \longrightarrow \mathcal{E} .
$$

Its kernel, denoted $\mathcal{N}_{\mathcal{E}}^{*}$, is locally free and $\mathbf{P}\left(\mathcal{N}_{\mathcal{E}}^{*}\right)$ is a smooth irreducible component of the incidence correspondence $\mathcal{Z}$ in (0.1). If the morphism in (0.4) is generically surjective or, equivalently, 


\section{INVARIANTS OF VECTOR BUNDLES}

$\mathcal{E}$ is generically generated by global sections, then dualizing the evaluation morphism we obtain a (rational) map

$$
\phi_{\mathcal{E}}: \mathbf{P}\left(\mathcal{E}^{*}\right)-->\mathbf{P}\left(H^{0}(\mathcal{E})^{*}\right) .
$$

This map can be viewed as a geometric realization of $\mathcal{E}$ which is dual to its geometric second Chern class. This 'duality' between $\mathcal{Z}_{f}$ and $\phi_{\mathcal{E}}$ gives a relationship between the degree of the cohomological invariant of $\mathcal{E}$ and some conventional properties of $\mathcal{E}$.

TheOREM (Theorem 2.9). Let $\mathcal{E}$ be generated by global sections and ample in the sense of Hartshorne. If the degree of the cohomological invariant of $\mathcal{E}$ is $\geqslant 2$ then $\operatorname{deg}\left(\phi_{\mathcal{E}}\right) \geqslant 2$.

The geometric consequences of the degree of the cohomological invariant (and $\phi_{\mathcal{E}}$ ) being $\geqslant 2$ are discussed at length in $\S 3$ (e.g. special properties of the zero-locus of a general section of $\mathcal{E}$ (Proposition 3.7), and properties of the family of incidence curves (Propositions 3.8 and 3.9, and Corollary 3.10)) so we will not develop this aspect further in the Introduction. What should be retained from the above discussion is that we have at our disposal an invariant living on $U_{f}$ and which is built from some cohomology data related to $\mathcal{E}$, and this invariant either recovers the geometric second Chern class of $\mathcal{E}$ or it 'sees' that $\mathcal{E}$ is special in a rather precise geometric manner.

Let us now describe the main points of construction of the cohomological invariant. The basic idea behind our invariant is to use $H^{1}\left(\mathcal{E}^{*}\right)$ to study the zero-loci of the global sections of $\mathcal{E}$, so it will always be assumed that $H^{1}\left(\mathcal{E}^{*}\right) \neq 0$.

We begin by considering a cohomological version of the incidence (0.1):

$$
Y=\left\{([e],[\xi]) \in \mathbf{P}\left(H^{0}(\mathcal{E})\right) \times \mathbf{P}\left(H^{1}\left(\mathcal{E}^{*}\right)\right) \mid e \cdot \xi=0 \text { in } H^{1}\left(\mathcal{O}_{X}\right)\right\}
$$

coming from the obvious pairing

$$
H^{0}(\mathcal{E}) \otimes H^{1}\left(\mathcal{E}^{*}\right) \longrightarrow H^{1}\left(\mathcal{O}_{X}\right) .
$$

For the second step of the construction we take $e \in U_{f}$, i.e. $Z_{e}=(e=0)$ is zero-dimensional, and observe that the linear space

$$
\tilde{Y}_{e}=\left\{\xi \in H^{1}\left(\mathcal{E}^{*}\right) \mid e \cdot \xi=0 \text { in } H^{1}\left(\mathcal{O}_{X}\right)\right\}
$$

can be canonically identified with a subspace of functions on $Z_{e}$, i.e. there is a natural inclusion $\tilde{Y}_{e} \hookrightarrow H^{0}\left(\mathcal{O}_{Z_{e}}\right)$. On the other hand one has the Grothendieck residue map (see [GH78b])

$$
\mathcal{R}_{e}: H^{0}\left(\mathcal{O}_{Z_{e}}\right) \longrightarrow H^{2}\left(\mathcal{O}_{X}(-L)\right),
$$

where $\mathcal{O}_{X}(-L)=\operatorname{det} \mathcal{E}^{*}$ (in other words $L=c_{1}(\mathcal{E})$ ). Using the multiplication in $H^{0}\left(\mathcal{O}_{Z_{e}}\right.$ ) we obtain the sequence of linear maps $S^{k} \tilde{Y}_{e} \longrightarrow H^{0}\left(\mathcal{O}_{Z_{e}}\right)$ which, composed with $\mathcal{R}_{e}$, yield

$$
\mathcal{R}_{k}(e): S^{k} \tilde{Y}_{e} \longrightarrow H^{2}\left(\mathcal{O}_{X}(-L)\right) .
$$

The invariants we propose are the sheafified versions of the maps $\mathcal{R}_{k}(e)$. Namely, the spaces $\tilde{Y}_{e}$ fit into a sheaf $\mathcal{H}_{\mathcal{E}}$ over $\mathbf{P}\left(H^{0}(\mathcal{E})\right)$, and the sheaf version of the Grothendieck residue becomes

$$
\mathcal{R}: p_{2 *} \mathcal{O}_{\mathcal{Z}_{f}} \longrightarrow H^{2}(-L) \otimes \mathcal{O}_{U_{f}}(-2) .
$$

Finally, the sheafification of $\mathcal{R}_{k}(e)$ yields

$$
\mathcal{R}_{k}: S^{k} \mathcal{H}_{\mathcal{E}} \otimes \mathcal{O}_{U_{f}}(-k) \longrightarrow H^{2}(-L) \otimes \mathcal{O}_{U_{f}}(-2)
$$

(see (1.9)). The sequence of morphisms $\mathcal{R}_{\mathcal{E}}=\left\{\mathcal{R}_{k}\right\}_{k \in \mathbb{N}}$ is what we call the cohomological invariant of $\mathcal{E}$ (see Definition 1.4). Let us point out that by construction $\mathcal{R}_{0}=\mathcal{R}_{1}=0$, while $\mathcal{R}_{2}$ comes from the well-known cup-product

$$
\gamma_{2}: S^{2} H^{1}\left(\mathcal{E}^{*}\right) \longrightarrow H^{2}\left(\wedge^{2} \mathcal{E}^{*}\right)=H^{2}\left(\mathcal{O}_{X}(-L)\right) .
$$




\section{REIDER}

So the morphisms $\mathcal{R}_{k}$, for $k \geqslant 3$, can be viewed as some kind of higher-order cohomological cupproducts.

The construction just outlined is rather formal and may strike one as artificial. However, if one accepts the idea that there should be an invariant of $\mathcal{E}$ that lifts $c_{2}(\mathcal{E})$ to $U_{f}$ and that this invariant should be based on some cohomology data associated to $\mathcal{E}$, one is naturally led to the cohomology cup-product (0.6). From this point on, the emergence of higher-order cohomology cup-products $\mathcal{R}_{k}, k \geqslant 3$, can be conceptually explained as higher-order derivatives of a natural deformation of the cup-product (0.6) as one varies the complex structure on $\mathcal{E}$. Roughly speaking what we are saying is that there is a 'Taylor series' for which $\mathcal{R}_{2}$ (or, equivalently, $\gamma_{2}$ ) is a 'constant term' and the morphisms $\mathcal{R}_{k}, k \geqslant 3$, are the 'coefficients' of the higher-order terms. Thus we are led to think of $U_{f}$ as the 'visible' part of a larger space and our 'Taylor series' should naturally live on this larger space.

To explain where such a larger space comes from, we observe that $\mathcal{E}$ admits a natural family of deformations parametrized by a variety which we will denote $\mathbf{J}$ (see $\S 4$ ). This variety has a structure of a projective bundle over $U_{f}$, where the fibre over $[e] \in U_{f}$ is $\mathbf{P}\left(\operatorname{Ext}^{1}\left(\mathcal{I}_{Z_{e}}(L), \mathcal{O}_{X}\right)\right)$, i.e. $\mathbf{J}$ parametrizes a family of torsion-free sheaves of rank 2 over $X$ having the Chern classes $\left(L, c_{2}(\mathcal{E})\right)$. By itself the variety $\mathbf{J}$ is rather uninteresting (it is a bundle of projective spaces over $U_{f}$ ). It is its 'modular' nature that makes it worthy of study.

The first feature of $\mathbf{J}$ is that it carries a distinguished divisor, denoted $\Xi$, which parametrizes the sheaves of the family which are not locally free. This divisor is a geometric reincarnation of the cohomological invariant $\mathcal{R}_{\mathcal{E}}$ : the divisor $\Xi$ determines $\pi: \mathcal{Z}_{f}^{\prime} \longrightarrow U_{f}$.

The second feature is that on the complement $\mathbf{J}^{\circ}=\mathbf{J} \backslash \Xi$ we have a 'universal' cup-product $\tilde{\gamma}_{2}$ (see $\S 4.3$ ). This is the 'Taylor series' we have mentioned above. It should be observed that $(\mathbf{J}, \Xi$ ) and $\tilde{\gamma}_{2}$ are the invariants of the incidence $p_{2}: \mathcal{Z}_{f} \longrightarrow U_{f}$ rather than those of $\mathcal{E}$. The presence of our bundle is detected by a distinguished section $s_{\mathcal{E}}: U_{f} \longrightarrow \stackrel{\circ}{\mathbf{J}}$. Identifying $U_{f}$ with its image $\Sigma=s_{\mathcal{E}}\left(U_{f}\right)$ we think of (0.6) as being defined on $\Sigma$. Then $\tilde{\gamma}_{2}$ restricted to $\Sigma$ coincides with (0.6), while the higher-order cup-products $\mathcal{R}_{k}, k \geqslant 3$, emerge as the higher-order derivatives of $\tilde{\gamma}_{2}$ taken along the directions of the normal bundle of $\Sigma$ in $\mathbf{J}$ and then evaluated on $\Sigma$. Observe that we can take derivatives of $\tilde{\gamma}_{2}$ in the fibre directions at any point of $\mathbf{J}$. Thus we have the 'universal' cohomological invariant defined on $\mathbf{J}$.

The 'universal' cohomological invariant gives rise to a (decreasing) filtration

$$
H^{0}\left(K_{X}+L\right) \otimes \mathcal{O}_{\mathbf{J}}=\tilde{\mathbf{F}}_{1} \supset \tilde{\mathbf{F}}_{2} \supset \cdots \supset \tilde{\mathbf{F}}_{k} \supset \cdots,
$$

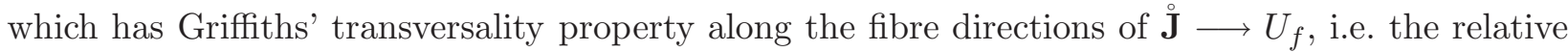
differential gives morphisms

$$
\mathcal{T}_{\mathbf{J} / U_{f}} \longrightarrow \mathcal{H} o m\left(\tilde{\mathbf{F}}_{k} / \tilde{\mathbf{F}}_{k+1}, \tilde{\mathbf{F}}_{k-1} / \tilde{\mathbf{F}}_{k}\right),
$$

where $\mathcal{T}_{\mathbf{j} / U_{f}}$ is the relative tangent bundle of $\stackrel{\circ}{\mathbf{J}} \longrightarrow U_{f}$. Thus we have associated to $\mathcal{E}$ in a canonical way the space $\mathbf{J}$ parametrizing some natural deformations of $\mathcal{E}$. On the one hand this space has the features of the classical Jacobian of curves, i.e. it carries a distinguished divisor $\Xi$ from which one can either recover the geometric second Chern class of $\mathcal{E}$ or see that $\mathcal{E}$ is special, and on the other hand the complement $\mathbf{J}=\mathbf{J} \backslash \Xi$ carries the universal cohomological invariant (which could be thought of as a non-Abelian version of theta-functions) giving rise to a variation of Hodge-like structures. This double nature of $\mathbf{J}$ seems to us to be of independent interest and deserves further investigation.

This paper is naturally divided into two parts. The first part $(\S \S 1-3)$ gives:

1) a 'formal' construction of the cohomological invariant of a rank-2 bundle $\mathcal{E}$; 


\section{INVARIANTS OF VECTOR BUNDLES}

2) a geometric interpretation of our invariant as well as a proof of the main property relating the cohomological invariant of $\mathcal{E}$ to its geometric second Chern class (see Proposition 2.3 and Theorem 2.9);

3) a relation of the degree of the cohomological invariant to the geometry of the map $\phi_{\mathcal{E}}$ in $(0.5)$.

Section 4 constitutes the second part of this paper. Here we give a much more conceptual interpretation of the cohomological invariant from the point of view of the deformation of a complex structure on a bundle. The variety of 'natural' deformations of $\mathcal{E}$ is introduced and we exhibit its dual nature: on the one hand it has the properties akin to the Jacobian of a curve (see $\S 4.1$, Remarks 4.2 and 4.6), and on the other hand it carries the 'universal' cohomological invariant $\mathbf{R}_{\mathbf{J}}$ (see $\S 4.2$ ) which gives rise to a variation of Hodge-like structures on the complement $\mathbf{J}=\mathbf{J} \backslash \Xi$ (e.g. the filtration (4.18) and its derivative possessing Griffiths' transversality property (see (4.19))).

In $\S 5$ the two filtrations arising from the cohomological invariant are discussed.

\section{Construction of new invariants}

Let $X$ be a smooth complex projective surface. We fix a line bundle $\mathcal{O}_{X}(L)$ and a positive integer $d \geqslant 1$. Let $\mathcal{E}$ be a vector bundle of rank 2 over $X$ with Chern classes $\left(c_{1}(\mathcal{E}), c_{2}(\mathcal{E})\right)$ such that $c_{1}(\mathcal{E})=L$ and $\operatorname{deg}\left(c_{2}(\mathcal{E})\right)=d$. Assuming that $h^{0}(\mathcal{E})$ and $h^{1}\left(\mathcal{E}^{*}\right) \geqslant 1$, we set $\mathbf{P}=\mathbf{P}\left(H^{0}(\mathcal{E})\right)$ and consider the morphism

$$
c: H^{1}\left(\mathcal{E}^{*}\right) \otimes \mathcal{O}_{\mathbf{P}}(-1) \longrightarrow H^{1}\left(\mathcal{O}_{X}\right) \otimes \mathcal{O}_{\mathbf{P}}
$$

defined at $[e] \in \mathbf{P}$ by the contraction map $H^{1}\left(\mathcal{E}^{*}\right) \stackrel{e}{\longrightarrow} H^{1}\left(\mathcal{O}_{X}\right)$.

Define $\mathcal{H}_{\mathcal{E}}(-1):=\operatorname{ker}(c)$. This is either zero or a second syzygy sheaf (see [OSS80]) so its rank is well defined.

Definition 1.1. The rank of $\mathcal{H}_{\mathcal{E}}(-1)$ will be called the index of speciality of $\mathcal{E}$ and it will be denoted by $\delta_{\mathcal{E}}$.

Remark 1.2. If $\delta_{\mathcal{E}} \geqslant 1$, then the singularity set of $\mathcal{H}_{\mathcal{E}}(-1)$ has codim $\geqslant 3$ ([OSS80, Theorem 1.1.6, p. 145]).

Assumption 1.3. From now on we will assume that $\mathcal{E}$ is subject to the following conditions:

1) there exists $e \in H^{0}(\mathcal{E})$ whose zero-locus is zero-dimensional;

2) $\delta_{\mathcal{E}} \geqslant 1$

3) $h^{1}\left(\operatorname{det} \mathcal{E}^{*}\right)=h^{1}(-L)=h^{0}(-L)=0$.

Consider the incidence correspondence

$$
X \times \mathbf{P} \supset \mathcal{Z}=\{(x, e) \mid e(x)=0\}
$$

and let $p_{i}(i=1,2)$ be the projection of $X \times \mathbf{P}$ on the $i$ th factor. Then $\mathcal{Z}$ can be viewed as the zero-locus of $\tilde{e} \in H^{0}\left(p_{1}{ }^{*} \mathcal{E} \otimes p_{2}{ }^{*} \mathcal{O}_{\mathbf{P}}(1)\right)$ corresponding to the identity endomorphism via the identifications

$$
H^{0}\left(p_{1}{ }^{*} \mathcal{E} \otimes p_{2}{ }^{*} \mathcal{O}_{\mathbf{P}}(1)\right)=H^{0}(X, \mathcal{E}) \otimes H^{0}\left(\mathbf{P}, \mathcal{O}_{\mathbf{P}}(1)\right)=H^{0}(X, \mathcal{E}) \otimes H^{0}(X, \mathcal{E})^{*}=\operatorname{End}\left(H^{0}(X, \mathcal{E})\right) .
$$

Consider the Koszul sequence defined by $\tilde{e}$,

$$
0 \longrightarrow p_{1}{ }^{*} \mathcal{O}(-L) \otimes p_{2}{ }^{*} \mathcal{O}_{\mathbf{P}}(-2) \stackrel{\tilde{e}}{\longrightarrow} p_{1}{ }^{*} \mathcal{E}^{*} \otimes p_{2}{ }^{*} \mathcal{O}_{\mathbf{P}}(-1) \stackrel{\tilde{e}}{\longrightarrow} \mathcal{I}_{\mathcal{Z}} \longrightarrow 0,
$$

where $\mathcal{I}_{\mathcal{Z}}$ is the sheaf of ideals of $\mathcal{Z}$. Let $U$ be the largest open subset of $\mathbf{P}$ parametrizing sections of $\mathcal{E}$ with a zero-dimensional zero-locus. By condition 1 of Assumption 1.3 the subset $U \neq \emptyset$. 


\section{REIDER}

Consider our incidence correspondence over $U$ :

$$
\mathcal{Z}_{U}=\mathcal{Z} \cap p_{2}{ }^{-1}(U) \stackrel{p_{2}}{\longrightarrow} U .
$$

This is a finite morphism of degree $d$. We will use the notation $Z_{e}$ for the fibre of $p_{2} \mid \mathcal{Z}_{U}$ over $[e] \in U$ as well as for the subscheme of zeros of $e \in H^{0}(\mathcal{E})$.

The Koszul sequence in (1.2) is exact over $\mathcal{Z}_{U}$. Combining it with the defining sequence for $\mathcal{Z}_{U}$,

$$
0 \longrightarrow \mathcal{I}_{\mathcal{Z}_{U}} \longrightarrow \mathcal{O}_{X \times U} \longrightarrow \mathcal{O}_{\mathcal{Z}_{U}} \longrightarrow 0,
$$

and taking the direct image with respect to $p_{2}$ we obtain the following diagram.

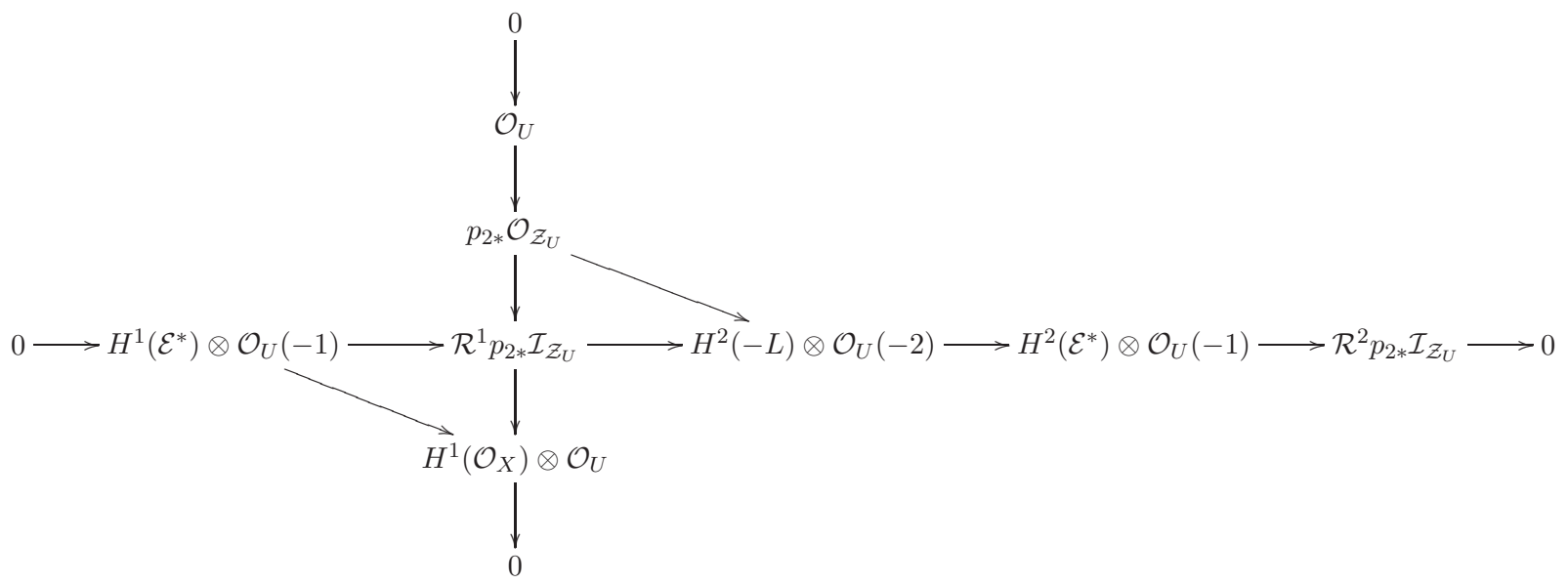

This gives us the morphism

$$
\mathcal{R}: p_{2 *} \mathcal{O}_{\mathcal{Z}_{U}} \longrightarrow H^{2}(-L) \otimes \mathcal{O}_{U}(-2) \text {. }
$$

Setting $\tilde{\mathcal{H}}_{\mathcal{E}}=\operatorname{ker} \mathcal{R}$ we obtain from (1.3) and the definition of $\mathcal{H}_{\mathcal{E}}(-1)$ the following exact sequence:

$$
0 \longrightarrow \mathcal{O}_{U} \longrightarrow \tilde{\mathcal{H}}_{\mathcal{E}} \longrightarrow \mathcal{H}_{\mathcal{E}}(-1) \longrightarrow 0
$$

We also have the trace morphism

$$
\operatorname{Tr}: p_{2 *} \mathcal{O}_{\mathcal{Z}_{U}} \longrightarrow \mathcal{O}_{U}
$$

Restricting it to $\tilde{\mathcal{H}}_{\mathcal{E}}$ and combining with (1.5) we obtain the following diagram.

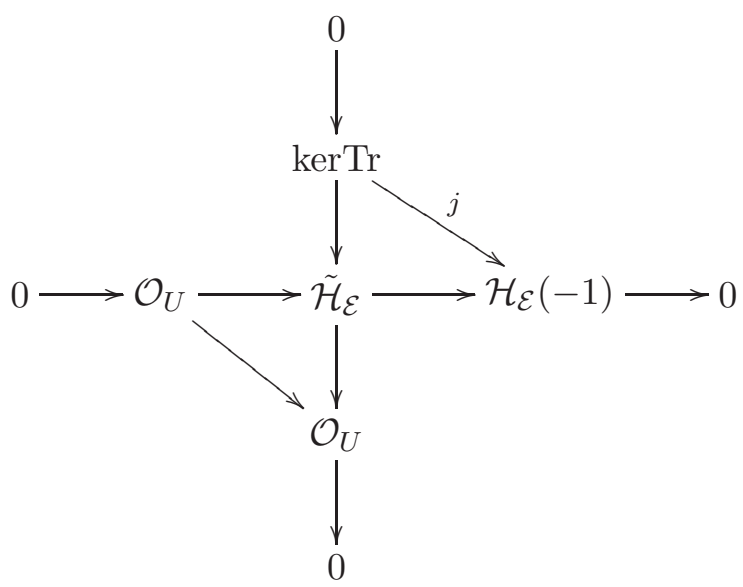

This induces the direct sum decomposition $\tilde{\mathcal{H}}_{\mathcal{E}}=\mathcal{O}_{U} \oplus$ kerTr. Identifying ker $\operatorname{Tr}$ with $\mathcal{H}_{\mathcal{E}}(-1)$ via the isomorphism $j$ we obtain

$$
\tilde{\mathcal{H}}_{\mathcal{E}}=\mathcal{O}_{U} \oplus \mathcal{H}_{\mathcal{E}}(-1)
$$




\section{INVARIANTS OF VECTOR BUNDLES}

Let $U^{\prime}$ be the largest open subset of $\mathbf{P}$ where $\mathcal{H}_{\mathcal{E}}(-1)$ is locally free. Put $U_{\mathcal{E}}=U \cap U^{\prime}$. From now on, unless stated otherwise, we will be working over $U_{\mathcal{E}}$. The incidence cycle over $U_{\mathcal{E}}$ will be denoted $\mathcal{Z}_{\mathcal{E}}$ (or simply $\mathcal{Z}$, if no confusion is likely).

Using the multiplicative structure of $p_{2 *} \mathcal{O}_{\mathcal{Z}}$ we have morphisms

$$
m_{k}: S^{k} \tilde{\mathcal{H}}_{\mathcal{E}} \longrightarrow p_{2 *} \mathcal{O}_{\mathcal{Z}_{\mathcal{E}}}
$$

for every $k \geqslant 0$. Composing this with $\mathcal{R}$ we obtain

$$
\tilde{\mathcal{R}}_{k}: S^{k} \tilde{\mathcal{H}}_{\mathcal{E}} \longrightarrow H^{2}(-L) \otimes \mathcal{O}_{U_{\mathcal{E}}}(-2) .
$$

The decomposition (1.6) implies that $S^{k}\left(\mathcal{H}_{\mathcal{E}}(-1)\right) \otimes \mathcal{O}_{U_{\mathcal{E}}}$ is the direct summand of $S^{k} \tilde{\mathcal{H}}_{\mathcal{E}}$. Restricting $\tilde{\mathcal{R}}_{k}$ to it we obtain the morphisms

$$
\mathcal{R}_{k}: S^{k} \mathcal{H}_{\mathcal{E}} \otimes \mathcal{O}_{U_{\mathcal{E}}}(-k) \longrightarrow H^{2}(-L) \otimes \mathcal{O}_{U_{\mathcal{E}}}(-2)
$$

for every $k \geqslant 0$.

Definition 1.4. The sequence of morphisms $\mathcal{R}_{\mathcal{E}}:=\left\{\mathcal{R}_{k}\right\}_{k \in \mathbb{N}}$ will be called the cohomological invariant of $\mathcal{E}$.

\section{Remark 1.5.}

a) By definition $\mathcal{R}_{0}=\mathcal{R}_{1}=0$, while for $k=2$ we have

$$
\begin{gathered}
\mathcal{R}_{2}(2): S^{2} \mathcal{H}_{\mathcal{E}} \longrightarrow H^{2}(-L) \otimes \mathcal{O}_{U_{\mathcal{E}}} \\
\bigcap_{S^{2} H^{1}\left(\mathcal{E}^{*}\right) \otimes \mathcal{O}_{U_{\mathcal{E}}}}
\end{gathered}
$$

and our morphism comes from the standard cup-product

$$
S^{2} H^{1}\left(\mathcal{E}^{*}\right) \longrightarrow H^{2}\left(\wedge^{2} \mathcal{E}^{*}\right)=H^{2}(-L) .
$$

For $k \geqslant 3$, the morphisms $\mathcal{R}_{k}$ seem to be new invariants of $\mathcal{E}$. They can be viewed as higherorder cohomological cup-products.

b) Tensoring (1.9) with $\mathcal{O}_{U_{\mathcal{E}}}(k)$ and considering the homomorphisms on the global sections we obtain the sequence of linear maps

$$
R_{k}^{0}: H^{0}\left(S^{k} \mathcal{H}_{\mathcal{E}}\right) \longrightarrow H^{2}(-L) \otimes H^{0}\left(\mathcal{O}_{U_{\mathcal{E}}}(k-2)\right)
$$

for every $k \in \mathbb{N}$.

c) If the irregularity $q(X)=h^{1}\left(\mathcal{O}_{X}\right)=0$, then $\mathcal{H}_{\mathcal{E}}=H^{1}\left(\mathcal{E}^{*}\right) \otimes \mathcal{O}_{\mathbf{P}}$ and we obtain the linear maps

$$
R_{k}^{0}: S^{k} H^{1}\left(\mathcal{E}^{*}\right) \longrightarrow H^{2}(-L) \otimes H^{0}\left(\mathcal{O}_{U_{\mathcal{E}}}(k-2)\right) .
$$

If, in addition, we assume that $\mathcal{E}$ is generated by global sections outside of a zero-dimensional subscheme, then $\mathbf{P} \backslash U_{\mathcal{E}}$ has codim $\geqslant 2$. By Hartog's theorem $H^{0}\left(\mathcal{O}_{U_{\mathcal{E}}}(k-2)\right)=H^{0}\left(\mathcal{O}_{\mathbf{P}}(k-2)\right)$ yielding the sequence of linear maps

$$
R_{k}^{0}: S^{k} H^{1}\left(\mathcal{E}^{*}\right) \longrightarrow H^{2}(-L) \otimes S^{k-2} H^{0}(\mathcal{E})^{*}
$$

for every $k \geqslant 2$.

The definition of the cohomological invariant implicitly contains a distinguished filtration of $H^{0}\left(K_{X}+L\right) \otimes \mathcal{O}_{U_{\mathcal{E}}}$ which we will now describe. 


\section{REIDER}

Let $\tilde{\mathcal{F}}_{k}$ be the kernel of $\left(\tilde{\mathcal{R}}_{k}(2)\right)^{*}$, the dual of $\tilde{\mathcal{R}}_{k}(2)$ (this is the morphism obtained by tensoring (1.8) with $\left.\mathcal{O}_{U_{\mathcal{E}}}(2)\right)$. Then for every $k \geqslant 2$ we have the following diagram.

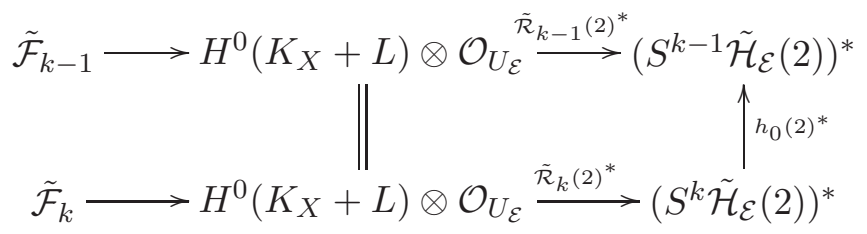

Here $h_{0}=1 \in H^{0}\left(\mathcal{O}_{U_{\mathcal{E}}}\right) \subset H^{0}\left(\tilde{\mathcal{H}}_{\mathcal{E}}\right)$ (see $(1.5)$ ) and the morphism $h_{0}(2)^{*}$ is dual to the morphism defined by the multiplication by $h_{0}$. This implies an inclusion $\tilde{\mathcal{F}}_{k} \hookrightarrow \tilde{\mathcal{F}}_{k-1}$, for every $k \geqslant 2$. Thus we obtain a decreasing filtration

$$
H^{0}\left(K_{X}+L\right) \otimes \mathcal{O}_{U_{\mathcal{E}}}=\tilde{\mathcal{F}}_{1} \supset \tilde{\mathcal{F}}_{2} \cdots \supset \tilde{\mathcal{F}}_{k-1} \supset \tilde{\mathcal{F}}_{k} \supset \cdots
$$

From (1.5) it follows that the kernel of the right hand column in (1.10) is

$$
\left(S^{k}\left(\mathcal{H}_{\mathcal{E}}(-1)\right)\right)^{*} \otimes \mathcal{O}_{U_{\mathcal{E}}}(-2) .
$$

This induces a morphism

$$
\tilde{\mathcal{F}}_{k-1} / \tilde{\mathcal{F}}_{k} \longrightarrow\left(S^{k}\left(\mathcal{H}_{\mathcal{E}}(-1)\right)\right)^{*} \otimes \mathcal{O}_{U_{\mathcal{E}}}(-2) .
$$

Tensoring with $\mathcal{O}_{U_{\mathcal{E}}}(2)$ and dualizing we obtain

$$
\left.\operatorname{gr}_{k}\left(\mathcal{R}_{\mathcal{E}}\right): S^{k} \mathcal{H}_{\mathcal{E}} \otimes \mathcal{O}_{U_{\mathcal{E}}}(-k)\right) \longrightarrow\left(\tilde{\mathcal{F}}_{k-1} / \tilde{\mathcal{F}}_{k}\right)^{*} \otimes \mathcal{O}_{U_{\mathcal{E}}}(-2), \quad \text { for } k \geqslant 2,
$$

the graded version of the morphisms $\mathcal{R}_{k}$ in (1.9). Observe that, while the definition of the $\mathcal{R}_{k}$ depends on a (canonical) identification of $\mathcal{H}_{\mathcal{E}}(-1)$ with a subsheaf of $\tilde{\mathcal{H}}_{\mathcal{E}}$, the graded morphisms are completely intrinsic.

It will be seen in $\S 4$ that the filtration (1.11) can be envisaged as a variation of Hodge-like structures and the morphisms in (1.12) are the consequences of Griffiths' transversality for this variation.

\section{Geometric interpretation of the cohomological invariant}

Let $\tilde{\mathcal{H}}_{\mathcal{E}}$ be as in $(1.6)$ and let $Y_{\mathcal{E}}=\mathbf{P}\left(\tilde{\mathcal{H}}_{\mathcal{E}}^{*}(-1)\right)$ with the natural projection $\pi: Y_{\mathcal{E}} \longrightarrow U_{\mathcal{E}}$. Take $\mathcal{O}_{Y_{\mathcal{E}}}(1)$ such that $\pi_{*} \mathcal{O}_{Y_{\mathcal{E}}}(1)=\tilde{\mathcal{H}}_{\mathcal{E}}(1)$. Observe that the incidence cycle $p_{2}: \mathcal{Z}_{\mathcal{E}} \longrightarrow U_{\mathcal{E}}$ admits the lifting $\kappa_{\mathcal{E}}$,

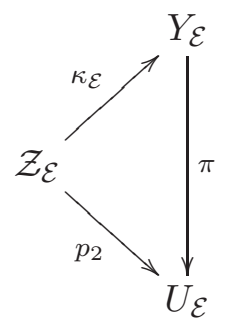

defined by the line bundle $\mathcal{L}=p_{2}{ }^{*} \mathcal{O}_{U_{\mathcal{E}}}(1)$ (see [Har77, Proposition 7.12, II]). We will relate the cohomological invariant $\mathcal{R}_{\mathcal{E}}$ to the properties of the morphism $\kappa_{\mathcal{E}}$.

Put $\mathcal{Z}^{\prime}=i m\left(\kappa_{\mathcal{E}}\right)$ and consider the exact sequence

$$
0 \longrightarrow \mathcal{I}_{\mathcal{Z}^{\prime}}(k) \longrightarrow \mathcal{O}_{Y_{\mathcal{E}}}(k) \longrightarrow \mathcal{O}_{\mathcal{Z}^{\prime}}(k) \longrightarrow 0
$$

where $\mathcal{I}_{\mathcal{Z}^{\prime}}$ is the sheaf of ideals of $\mathcal{Z}^{\prime}$ in $Y_{\mathcal{E}}$. Taking the direct image with respect to $\pi$ we obtain

$$
0 \longrightarrow \pi_{*}\left(\mathcal{I}_{\mathcal{Z}^{\prime}}(k)\right) \longrightarrow S^{k}\left(\tilde{\mathcal{H}}_{\mathcal{E}}(1)\right) \longrightarrow \pi_{*}\left(\mathcal{O}_{\mathcal{Z}^{\prime}}(k)\right)
$$




\section{INVARIANTS OF VECTOR BUNDLES}

On the other hand we have the inclusion

$$
\mathcal{O}_{\mathcal{Z}^{\prime}}(k) \longrightarrow \kappa_{\mathcal{E} *}\left(\mathcal{O}_{\mathcal{Z}}\right) \otimes \mathcal{O}_{\mathcal{Z}^{\prime}}(k)=\kappa_{\mathcal{E} *}\left(\kappa_{\mathcal{E}}{ }^{*} \mathcal{O}_{Y_{\mathcal{E}}}(k)\right)=\kappa_{\mathcal{E} *}\left(p_{2}{ }^{*} \mathcal{O}_{U_{\mathcal{E}}}(k)\right) .
$$

Its direct image with respect to $\pi$ gives an inclusion

$$
\pi_{*}\left(\mathcal{O}_{\mathcal{Z}^{\prime}}(k)\right) \longrightarrow p_{2 *}\left(\mathcal{O}_{\mathcal{Z}_{\mathcal{E}}}\right) \otimes \mathcal{O}_{U_{\mathcal{E}}}(k) .
$$

Combining this with (2.1) we obtain the sequence

$$
0 \longrightarrow \pi_{*}\left(\mathcal{I}_{\mathcal{Z}^{\prime}}(k)\right) \longrightarrow S^{k}\left(\tilde{\mathcal{H}}_{\mathcal{E}}(1)\right) \longrightarrow p_{2 *}\left(\mathcal{O}_{\mathcal{Z}_{\mathcal{E}}}\right) \otimes \mathcal{O}_{U_{\mathcal{E}}}(k)
$$

where the morphism on the right is the multiplication morphism $m_{k}$ (see (1.7)) tensored with $\mathcal{O}_{U_{\mathcal{E}}}(k)$. This implies that the kernel of $\tilde{\mathcal{R}}_{k}$ fits into the following exact sequence:

$$
0 \longrightarrow \pi_{*}\left(\mathcal{I}_{\mathcal{Z}^{\prime}}(k)\right) \otimes \mathcal{O}_{U_{\mathcal{E}}}(-k) \longrightarrow \operatorname{ker} \tilde{\mathcal{R}}_{k} \longrightarrow \tilde{\mathcal{H}}_{\mathcal{E}}
$$

Next we show that the morphism on the right is surjective. Let $h_{0}$ be the section of $\tilde{\mathcal{H}}_{\mathcal{E}}$ which is equal to 1 everywhere on $U_{\mathcal{E}}$. The multiplication by $h_{0}$ induces an injective morphism

$$
\tilde{\mathcal{H}}_{\mathcal{E}} \stackrel{h_{0}^{k-1}}{\longrightarrow} S^{k} \tilde{\mathcal{H}}_{\mathcal{E}}
$$

and its image is clearly in ker $\tilde{\mathcal{R}}_{k}$. This yields the following diagram.

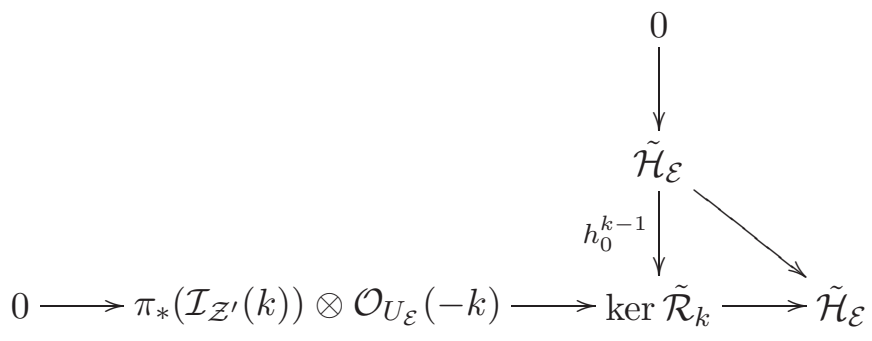

The induced morphism given by the slanted arrow in the above diagram is the identity. This gives us not only surjectivity in (2.2) but also a distinguished decomposition

$$
\operatorname{ker} \tilde{\mathcal{R}}_{k}=\pi_{*}\left(\mathcal{I}_{\mathcal{Z}^{\prime}}(k)\right) \otimes \mathcal{O}_{U_{\mathcal{E}}}(-k) \oplus \tilde{\mathcal{H}}_{\mathcal{E}}
$$

So the cohomological invariant of $\mathcal{E}$ comes quite close to recovering the image of the morphism $\kappa_{\mathcal{E}}$. More precisely, given the cohomological invariant $\mathcal{R}_{\mathcal{E}}=\left\{\mathcal{R}_{k}\right\}_{k \in \mathbb{N}}$, define

$$
\tilde{\mathcal{H}}_{\mathcal{E}}=\mathcal{O}_{U_{\mathcal{E}}} \oplus \mathcal{H}_{\mathcal{E}}(-1) \text {. }
$$

The decomposition $S^{k} \tilde{\mathcal{H}}_{\mathcal{E}}=\bigoplus_{i=0}^{k} S^{i}\left(\mathcal{H}_{\mathcal{E}}(-1)\right)$ gives us morphisms

$$
\tilde{\mathcal{R}}_{k}: S^{k} \tilde{\mathcal{H}}_{\mathcal{E}} \longrightarrow H^{2}(-L) \otimes \mathcal{O}_{U_{\mathcal{E}}}(-2),
$$

where $\tilde{\mathcal{R}}_{k}=\sum_{i=0}^{k} \mathcal{R}_{i}$.

Lemma 2.1. The sequence of morphisms $\left\{\tilde{\mathcal{R}}_{k}\right\}_{k \in \mathbb{N}}$ defines a sheaf $\mathcal{J}_{\mathcal{E}}=\bigoplus_{k \in \mathbb{N}} \mathcal{J}_{\mathcal{E}}^{k}$ of homogeneous ideals in $S^{\bullet} \tilde{\mathcal{H}}_{\mathcal{E}}$ having the following properties:

1) $\mathcal{J}_{\mathcal{E}}^{k} \supseteq \pi_{*}\left(\mathcal{I}_{\mathcal{Z}^{\prime}}(k)\right) \otimes \mathcal{O}_{U_{\mathcal{E}}}(-k)$, for every $k \in \mathbb{N}$;

2) for every $k, l \in \mathbb{N}$, there exist morphisms

$$
\psi_{k, l}: \mathcal{J}_{\mathcal{E}}^{k} \otimes S^{l} \tilde{\mathcal{H}}_{\mathcal{E}} \longrightarrow \tilde{\mathcal{H}}_{\mathcal{E}}
$$

induced by the multiplication in $S^{\bullet} \tilde{\mathcal{H}}_{\mathcal{E}}$. 


\section{REIDER}

Proof. We define $\mathcal{J}_{\mathcal{E}}^{k}$ as the limit of a certain decreasing filtration of $S^{k} \tilde{\mathcal{H}}_{\mathcal{E}}$. This filtration is defined as follows. Put $\Lambda_{k}^{(0)}=\operatorname{ker} \tilde{\mathcal{R}}_{k}$, for every $k \in \mathbb{N}$, and consider the morphisms

$$
\alpha_{k}^{(0)}: \Lambda_{k}^{(0)} \longrightarrow \tilde{\mathcal{H}}_{\mathcal{E}}^{*} \otimes\left(S^{k+1} \tilde{\mathcal{H}}_{\mathcal{E}} / \Lambda_{k+1}^{(0)}\right)
$$

induced by the multiplication in $S^{\bullet} \tilde{\mathcal{H}}_{\mathcal{E}}$. Put $\Lambda_{k}^{(1)}=\operatorname{ker} \alpha_{k}^{(0)}$, for every $k \in \mathbb{N}$. We obviously have $\Lambda_{k}^{(1)} \subset \Lambda_{k}^{(0)}$ and $\Lambda_{k}^{(1)} \otimes \tilde{\mathcal{H}}_{\mathcal{E}} \longrightarrow \Lambda_{k+1}^{(0)}$, for every $k \in \mathbb{N}$. This induces the morphisms

$$
\alpha_{k}^{(1)}: \Lambda_{k}^{(1)} \longrightarrow \tilde{\mathcal{H}}_{\mathcal{E}}^{*} \otimes\left(\Lambda_{k+1}^{(0)} / \Lambda_{k+1}^{(1)}\right) .
$$

Putting $\Lambda_{k}^{(2)}=\operatorname{ker} \alpha_{k}^{(1)}$, for every $k \in \mathbb{N}$, and continuing in the same fashion, we obtain a decreasing filtration

with morphisms

$$
\operatorname{ker} \tilde{\mathcal{R}}_{k}=\Lambda_{k}^{(0)} \supseteq \cdots \supseteq \Lambda_{k}^{(m)} \supseteq \Lambda_{k}^{(m+1)} \supseteq \cdots
$$

$$
\alpha_{k}^{(m)}: \Lambda_{k}^{(m)} \longrightarrow \tilde{\mathcal{H}}_{\mathcal{E}}^{*} \otimes\left(\Lambda_{k+1}^{(m-1)} / \Lambda_{k+1}^{(m)}\right) .
$$

We define $\mathcal{J}_{\mathcal{E}}^{k}$ to be the limit of the above filtration and set $\mathcal{J}_{\mathcal{E}}=\oplus_{k \in \mathbb{N}} \mathcal{J}_{\mathcal{E}}^{k}$. To see that it is a sheaf of ideals in $S^{\bullet} \tilde{\mathcal{H}}_{\mathcal{E}}$ it is enough to show that $\mathcal{J}_{\mathcal{E}}^{k} \otimes \tilde{\mathcal{H}}_{\mathcal{E}} \longrightarrow \mathcal{J}_{\mathcal{E}}^{k+1}$. Let $f$ be a local section of $\mathcal{J}_{\mathcal{E}}^{k}$. By definition $f \cdot t$ is a local section of $\Lambda_{k+1}^{(m)}$, for every $m \in \mathbb{N}$ and every local section $t$ of $\tilde{\mathcal{H}}_{\mathcal{E}}$. Hence, $f \cdot t$ is a local section of $\mathcal{J}_{\mathcal{E}}^{k+1}$.

To show part 1 of the lemma we need to prove that $\pi_{*}\left(\mathcal{I}_{\mathcal{Z}^{\prime}}(k)\right) \otimes \mathcal{O}_{U_{\mathcal{E}}}(-k)$ is contained in $\Lambda_{k}^{(m)}$, for all $m \in \mathbb{N}$. This can be seen by induction on $m$. For $m=0$, the inclusion

$$
\pi_{*}\left(\mathcal{I}_{\mathcal{Z}^{\prime}}(k)\right) \otimes \mathcal{O}_{U_{\mathcal{E}}}(-k) \subset \Lambda_{k}^{(0)}
$$

follows from (2.3) and it holds for every $k \in \mathbb{N}$. Assume that $\pi_{*}\left(\mathcal{I}_{\mathcal{Z}^{\prime}}(k)\right) \otimes \mathcal{O}_{U_{\mathcal{E}}}(-k) \subset \Lambda_{k}^{(m)}$ holds for every $k \in \mathbb{N}$. Observe that for every $k \in \mathbb{N}$ we have

$$
\pi_{*}\left(\mathcal{I}_{\mathcal{Z}^{\prime}}(k)\right) \otimes \mathcal{O}_{U_{\mathcal{E}}}(-k) \otimes \tilde{\mathcal{H}}_{\mathcal{E}} \longrightarrow \pi_{*}\left(\mathcal{I}_{\mathcal{Z}^{\prime}}(k+1)\right) \otimes \mathcal{O}_{U_{\mathcal{E}}}(-k-1) \subset \Lambda_{k+1}^{(m)} .
$$

This implies that $\pi_{*}\left(\mathcal{I}_{\mathcal{Z}^{\prime}}(k)\right) \otimes \mathcal{O}_{U_{\mathcal{E}}}(-k) \subset \Lambda_{k}^{(m+1)}$.

Turning to the second assertion of the lemma, observe that we have a morphism $\Lambda_{k}^{(0)}=$ ker $\tilde{\mathcal{R}}_{k} \longrightarrow \tilde{\mathcal{H}}_{\mathcal{E}}$ as in $(2.2)$. This induces the morphism $\mathcal{J}_{\mathcal{E}}^{k} \longrightarrow \tilde{\mathcal{H}}_{\mathcal{E}}$, for every $k \in \mathbb{N}$. Since $\mathcal{J}_{\mathcal{E}}$ is the sheaf of homogeneous ideals, we have the morphisms

$$
\mathcal{J}_{\mathcal{E}}^{k} \otimes S^{l} \tilde{\mathcal{H}}_{\mathcal{E}} \longrightarrow \mathcal{J}_{\mathcal{E}}^{k+l} \longrightarrow \tilde{\mathcal{H}}_{\mathcal{E}}
$$

asserted in the lemma.

Set

$$
\mathcal{M}_{\mathcal{E}}=\bigoplus_{k \geqslant 0} \pi_{*}\left(\mathcal{I}_{\mathcal{Z}^{\prime}}(k)\right) \otimes \mathcal{O}_{U_{\mathcal{E}}}(-k)
$$

In the case of equality in Lemma 2.1, part 1, the cohomological invariant of $\mathcal{E}$ determines this module and hence the image of $\kappa_{\mathcal{E}}$. In general, the module $\mathcal{J}_{\mathcal{E}}$ described in Lemma 2.1 is larger, so $\mathcal{R}_{\mathcal{E}}$ determines a subscheme of $\mathcal{Z}^{\prime}$ which will be denoted $\mathcal{Z}^{\prime}\left(\mathcal{R}_{\mathcal{E}}\right)$. Furthermore, the condition 2 of Lemma 2.1 says that the complement of the pullback $\kappa_{\mathcal{E}}^{*}\left(\mathcal{Z}^{\prime}\left(\mathcal{R}_{\mathcal{E}}\right)\right)$ is a family of zero-dimensional subschemes of $X$ which are $L$-special (recall (see [Tyu87]) that a zero-dimensional subscheme $Z \subset X$ is called $L$-special if the restriction map $\rho_{Z}: H^{0}\left(\mathcal{O}_{X}\left(K_{X}+L\right)\right) \longrightarrow H^{0}\left(\mathcal{O}_{Z}\left(K_{X}+L\right)\right)$ fails to be surjective; $\delta(L, Z):=\operatorname{deg} Z-\operatorname{rk} \rho_{Z}$ is called the index of $L$-speciality of $Z$ ). We formalize the above situation in the following definition. 


\section{INVARIANTS OF VECTOR BUNDLES}

\section{DeFinition 2.2.}

1) $\mathcal{E}$ is called cycle-decomposable (or, equivalently, $\mathcal{E}$ admits a cycle-decomposition), if the projection $p_{2}: \mathcal{Z}_{\mathcal{E}} \longrightarrow U_{\mathcal{E}}$ admits a nontrivial factorization

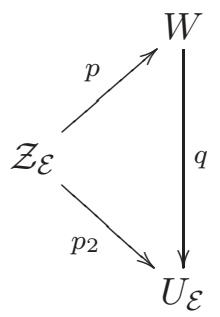

where $p$ and $q$ are finite surjective (not equal to identity) morphisms.

2) We say that $\mathcal{E}$ admits an $L$-special cycle-decomposition if it is cycle-decomposable and for general $[e] \in U_{\mathcal{E}}$ the scheme $W_{e}=q^{*}([e])$ has the following property: there is a nonempty subscheme $W_{e}^{\prime} \subset W_{e}$ such that $p^{*} A$ is $L$-special for every nonempty subscheme $A \subset W_{e}^{\prime}$.

Proposition 2.3. The cohomological invariant $\mathcal{R}_{\mathcal{E}}$ determines the image of $\kappa_{\mathcal{E}}$ unless $\mathcal{E}$ admits an $L$-special decomposition given by the following diagram.

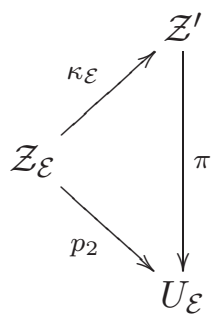

More precisely, the cohomological invariant determines the subscheme $\mathcal{Z}^{\prime}\left(\mathcal{R}_{\mathcal{E}}\right)$ and the fibres of the complement $\mathcal{Z}^{\prime} \backslash \mathcal{Z}^{\prime}\left(\mathcal{R}_{\mathcal{E}}\right)$ are subject to the property 2 of Definition 2.2 .

Proof. Let $\mathcal{Z}^{\prime}\left(\mathcal{R}_{\mathcal{E}}\right)$ be the subscheme of $\mathcal{Z}^{\prime}$ defined by the sheaf $\mathcal{J}_{\mathcal{E}}$ of Lemma 2.1 and let $Z_{e}^{\prime}\left(\mathcal{R}_{\mathcal{E}}\right)$ be the fibre of $\mathcal{Z}^{\prime}\left(\mathcal{R}_{\mathcal{E}}\right)$ over $[e] \in U_{\mathcal{E}}$. Then we have $\mathcal{J}_{\mathcal{E},[e]}^{k}=H^{0}\left(\mathcal{I}_{Z_{e}^{\prime}\left(\mathcal{R}_{\mathcal{E}}\right)}(k)\right)$. If $\mathcal{R}_{\mathcal{E}}$ fails to determine $\mathcal{Z}^{\prime}$ then $H^{0}\left(\mathcal{I}_{Z_{e}^{\prime}\left(\mathcal{R}_{\mathcal{E}}\right)}(k)\right) \neq H^{0}\left(\mathcal{I}_{Z_{e}^{\prime}}(k)\right)$, for all $k \gg 0$. Furthermore, by Lemma 2.1 we also have homomorphisms

for all $k, l \in \mathbb{N}$.

$$
H^{0}\left(\mathcal{I}_{Z_{e}^{\prime}\left(\mathcal{R}_{\mathcal{E}}\right)}(k)\right) \otimes S^{l} \tilde{\mathcal{H}}_{\mathcal{E},[e]} \longrightarrow \tilde{\mathcal{H}}_{\mathcal{E},[e]}
$$

Let $Z_{e}^{\prime \prime}$ be the subscheme of $Z_{e}^{\prime}$ residual to $Z_{e}^{\prime}\left(\mathcal{R}_{\mathcal{E}}\right)$. We claim that $Z_{e}^{\prime \prime}$ has the property of Definition 2.2, part 2. In fact, take $l \gg 0$ such that the map

$$
S^{l} \tilde{\mathcal{H}}_{\mathcal{E},[e]} \longrightarrow H^{0}\left(\mathcal{O}_{Z_{e}^{\prime \prime}}(l)\right)
$$

is surjective. Then for every nonempty subscheme $A \subset Z_{e}^{\prime \prime}$ we can find $F \in S^{l} \tilde{\mathcal{H}}_{\mathcal{E},[e]}$ such that $F$ is an invertible section of $\mathcal{O}_{A}(l)$ and vanishes on $\left(Z^{\prime \prime} \backslash A\right)$. Consider the homomorphism (2.5) restricted to the subspace $H^{0}\left(\mathcal{I}_{Z_{e}^{\prime}\left(\mathcal{R}_{\mathcal{E}}\right)}(k)\right) \otimes \mathbf{C} F$. It is clear that the image is contained in $\tilde{\mathcal{H}}_{\mathcal{E},[e]} \cap H^{0}\left(\mathcal{I}_{Z_{e} \backslash \kappa_{\mathcal{E}}^{*}(A)}\right)$ and that for $k \gg 0$ the image is nonzero. Thus we obtain $\tilde{\mathcal{H}}_{\mathcal{E},[e]} \cap H^{0}\left(\mathcal{I}_{Z_{e} \backslash \kappa_{\mathcal{E}}^{*}(A)}\right) \neq 0$, for every nonempty subscheme $A \subset Z_{e}^{\prime \prime}$. The $L$-speciality of $\kappa_{\mathcal{E}}^{*}(A)$ can now be seen from the following claim.

Claim 2.4. The cokernel of

$$
\rho_{\kappa_{\mathcal{E}}^{*}(A)}: H^{0}\left(K_{X}+L\right) \longrightarrow H^{0}\left(\mathcal{O}_{\kappa_{\mathcal{E}}^{*}(A)}\left(K_{X}+L\right)\right)
$$

is isomorphic to $\tilde{\mathcal{H}}_{\mathcal{E},[e]} \cap H^{0}\left(\mathcal{I}_{Z_{e} \backslash \kappa_{\mathcal{E}}^{*}(A)}\right)$. In particular, the index of speciality of $\kappa_{\mathcal{E}}^{*}(A)$ is equal to $\operatorname{dim}\left(\tilde{\mathcal{H}}_{\mathcal{E},[e]} \cap H^{0}\left(\mathcal{I}_{Z_{e} \backslash \kappa_{\mathcal{E}}^{*}(A)}\right)\right)$. 


\section{REIDER}

Proof. Let $Z$ be a zero-dimensional subscheme of $X$. The group of extensions $\operatorname{Ext}^{1}\left(\mathcal{I}_{Z}, \mathcal{O}_{X}(-L)\right)$ is computed from the following exact sequence (see [GH78a]).

$$
0 \longrightarrow \operatorname{Ext}^{1}\left(\mathcal{I}_{Z}, \mathcal{O}_{X}(-L)\right) \longrightarrow \operatorname{Ext}^{2}\left(\mathcal{O}_{Z}, \mathcal{O}_{X}(-L)\right) \longrightarrow H^{2}(-L)
$$

By Serre duality this is dual to

$$
H^{0}\left(\mathcal{O}_{X}\left(K_{X}+L\right)\right) \stackrel{\rho_{Z}}{\longrightarrow} H^{0}\left(\mathcal{O}_{Z}\left(K_{X}+L\right)\right) \longrightarrow H^{1}\left(\mathcal{I}_{Z}\left(K_{X}+L\right)\right) .
$$

Given an extension class $\alpha \in \operatorname{Ext}^{1}\left(\mathcal{I}_{Z}, \mathcal{O}_{X}(-L)\right)$ we have the cup-product

$$
H^{0}\left(\mathcal{O}_{Z}\right) \stackrel{\alpha}{\longrightarrow} \operatorname{Ext}^{2}\left(\mathcal{O}_{Z}, \mathcal{O}_{X}(-L)\right)=H^{0}\left(\mathcal{O}_{Z}\left(K_{X}+L\right)\right)^{*}
$$

coming from the Yoneda pairing

$$
\operatorname{Ext}^{0}\left(\mathcal{O}_{Z}, \mathcal{O}_{Z}\right) \otimes \operatorname{Ext}^{2}\left(\mathcal{O}_{Z}, \mathcal{O}_{X}(-L)\right) \longrightarrow \operatorname{Ext}^{2}\left(\mathcal{O}_{Z}, \mathcal{O}_{X}(-L)\right)
$$

and the inclusions

$$
\begin{aligned}
H^{0}\left(\mathcal{O}_{Z}\right) \otimes \operatorname{Ext}^{1}\left(\mathcal{I}_{Z}, \mathcal{O}_{X}(-L)\right) & \hookrightarrow H^{0}\left(\mathcal{O}_{Z}\right) \otimes \operatorname{Ext}^{2}\left(\mathcal{O}_{Z}, \mathcal{O}_{X}(-L)\right) \\
& \hookrightarrow \operatorname{Ext}^{0}\left(\mathcal{O}_{Z}, \mathcal{O}_{Z}\right) \otimes \operatorname{Ext}^{2}\left(\mathcal{O}_{Z}, \mathcal{O}_{X}(-L)\right) .
\end{aligned}
$$

Furthermore, if $\alpha$ corresponds to a locally free sheaf, then the cup-product with $\alpha$ is an isomorphism.

Applying the above considerations to $Z_{e}$ and to the extension class $\alpha=\left(\mathcal{E}^{*}, e\right)$ given by the Koszul sequence

$$
0 \longrightarrow \mathcal{O}_{X}(-L) \stackrel{e}{\longrightarrow} \mathcal{E}^{*} \stackrel{e}{\longrightarrow} \mathcal{I}_{Z_{e}} \longrightarrow 0,
$$

we obtain an isomorphism $H^{0}\left(\mathcal{O}_{Z_{e}}\right) \stackrel{\alpha}{\longrightarrow} H^{0}\left(\mathcal{O}_{Z_{e}}\left(K_{X}+L\right)\right)^{*}$. Composing it with the dual of $\rho_{Z_{e}}$ gives us the morphism $\mathcal{R}_{e}$ (see (1.4)). In particular, we have the diagram

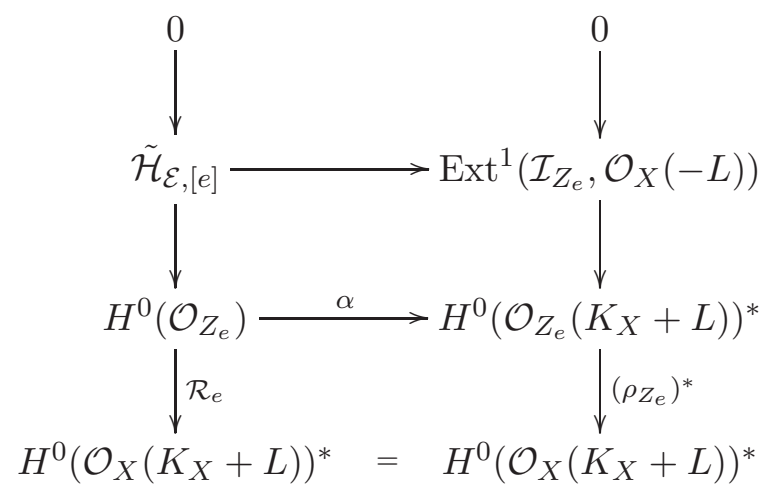

inducing an isomorphism $\tilde{\mathcal{H}}_{\mathcal{E},[e]} \stackrel{\alpha}{\longrightarrow} \operatorname{Ext}^{1}\left(\mathcal{I}_{Z_{e}}, \mathcal{O}_{X}(-L)\right)$.

If $Z_{1}$ is a subscheme of $Z_{e}$ and $Z_{2}$ is its residual subscheme, then we have

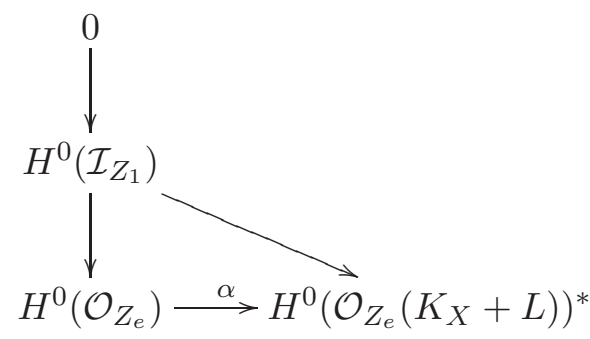




\section{INVARIANTS OF VECTOR BUNDLES}

where the slanted arrow factors through $H^{0}\left(\mathcal{O}_{Z_{2}}\left(K_{X}+L\right)\right)^{*}$. This yields the commutative diagram

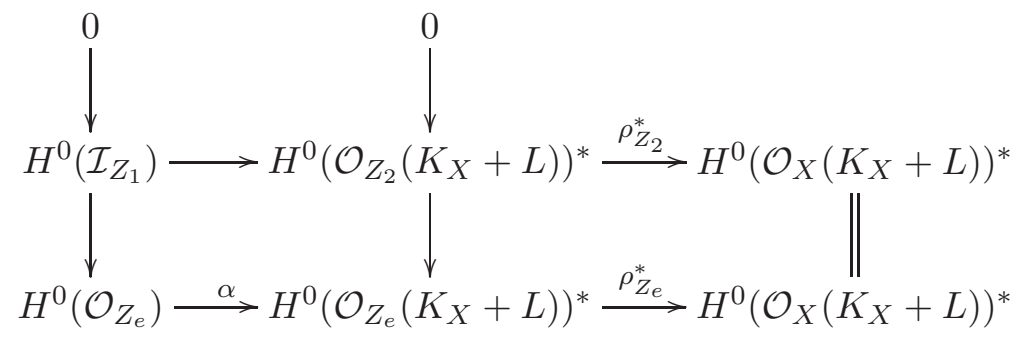

from which it follows that $\operatorname{ker}\left(\rho_{Z_{2}}^{*}\right)=\tilde{\mathcal{H}}_{\mathcal{E},[e]} \cap H^{0}\left(\mathcal{I}_{Z_{1}}\right)$. Taking $Z_{1}=Z_{e} \backslash \kappa_{\mathcal{E}}^{*}(A)$ we obtain the assertion of the claim.

This completes the proof of Proposition 2.3.

Definition 2.5. The degree of $\kappa_{\mathcal{E}}$ (whenever defined) will be called the degree of the cohomological invariant $\mathcal{R}_{\mathcal{E}}$ and denoted by $\operatorname{deg} \mathcal{R}_{\mathcal{E}}$.

TheOREM 2.6. If $\mathcal{Z}_{\mathcal{E}}$ is irreducible then the cohomological invariant of $\mathcal{R}_{\mathcal{E}}$ determines $\mathcal{Z}^{\prime}$ (as in Proposition 2.3) unless $\mathcal{R}_{\mathcal{E}}=0$. In the latter case $d=\operatorname{deg}\left(c_{2}(\mathcal{E})\right)=\left(\operatorname{deg} \mathcal{R}_{\mathcal{E}}\right)\left(\delta_{\mathcal{E}}+1\right)$, where $\delta_{\mathcal{E}}$ is the index of speciality of $\mathcal{E}$ (Definition 1.1).

Proof. From the irreducibility of $\mathcal{Z}_{\mathcal{E}}$ it follows that the subscheme $\mathcal{Z}^{\prime}\left(\mathcal{R}_{\mathcal{E}}\right)$ in Proposition 2.3 is either $\mathcal{Z}^{\prime}$ or empty. The latter case implies that the sheaf of ideals $\mathcal{J}_{\mathcal{E}}$ in Lemma 2.1 coincides with the ring $S^{\bullet} \tilde{\mathcal{H}}_{\mathcal{E}}$. This combined with Lemma 2.1, part 2, implies that $\tilde{\mathcal{H}}_{\mathcal{E}}$ is a subring of $p_{2 *}\left(\mathcal{O}_{\mathcal{Z}_{\mathcal{E}}}\right)$. This yields $\operatorname{deg} \pi=\operatorname{rk}\left(\pi_{*}\left(\mathcal{O}_{\mathcal{Z}^{\prime}}\right)\right)=\delta_{\mathcal{E}}+1$, where the last equality follows from (1.5). Substituting this in $d=\operatorname{deg}\left(c_{2}(\mathcal{E})\right)=\operatorname{deg} \mathcal{R}_{\mathcal{E}} \operatorname{deg} \pi$, we obtain the assertion.

Corollary 2.7. Let $\mathcal{Z}_{\mathcal{E}}$ be smooth and irreducible. If $\mathcal{R}_{\mathcal{E}}$ fails to determine $\mathcal{Z}^{\prime}$, then for general $e \in H^{0}(\mathcal{E})$ its scheme of zeros has the decomposition

$$
Z_{e}=\sum_{p^{\prime} \in Z_{e}^{\prime}} \kappa_{\mathcal{E}}^{*}\left(p^{\prime}\right)
$$

where $Z_{e}^{\prime}=\kappa_{\mathcal{E}}\left(Z_{e}\right)$ has length $\left(\delta_{\mathcal{E}}+1\right)$ and the $\kappa_{\mathcal{E}}^{*}\left(p^{\prime}\right)$ are $L$-special subschemes of length $\operatorname{deg} \mathcal{R}_{\mathcal{E}}$ and index of speciality $\delta\left(L, \kappa_{\mathcal{E}}^{*}\left(p^{\prime}\right)\right)=1$.

Proof. From the smoothness hypothesis it follows that for a general section $e$ of $\mathcal{E}$ its zero-scheme $Z_{e}$ is $d$ distinct points. From Theorem 2.6 we deduce that $Z_{e}^{\prime}$ is a set of $\left(\delta_{\mathcal{E}}+1\right)$ distinct points. Applying Proposition 2.3 to points in $Z_{e}^{\prime}$ we obtain that $\kappa_{\mathcal{E}}^{*}\left(p^{\prime}\right)$ is $L$-special, for every $p^{\prime} \in Z_{e}^{\prime}$. Finally, the index of $L$-speciality of $\kappa_{\mathcal{E}}^{*}\left(p^{\prime}\right)$ is obtained by counting the number of hyperplanes in $\mathbf{P}\left(\left(\tilde{\mathcal{H}}_{\mathcal{E},[e]}\right)^{*}\right)$ passing through $Z_{e}^{\prime} \backslash\left\{p^{\prime}\right\}$. Since $Z_{e}^{\prime} \backslash\left\{p^{\prime}\right\}$ must span a subspace of codimension 1, we obtain $\delta\left(L, \kappa_{\mathcal{E}}^{*}\left(p^{\prime}\right)\right)=1$.

The case of vanishing of the cohomological invariant, as Corollary 2.7 indicates, is a rather special situation. It could be viewed as some kind of 'hyperellipticity' of vector bundles of rank 2 . This case will be treated in more detail elsewhere (however, see Theorem 3.11 and Corollary 3.13). For now we will be concerned with the 'general' case of $\operatorname{deg} \mathcal{R}_{\mathcal{E}}=1$. Namely, we will give some sufficient conditions for the cohomological invariant to have degree 1. 


\section{REIDER}

Let $\mathcal{E}$ be generated by global sections. Then we have the exact sequence

$$
0 \longrightarrow \mathcal{N}_{\mathcal{E}}^{*} \longrightarrow H^{0}(\mathcal{E}) \otimes \mathcal{O}_{X} \longrightarrow \mathcal{E} \longrightarrow 0,
$$

where the morphism on the right is the evaluation map. The projectivized bundle $\mathbf{P}\left(\mathcal{N}_{\mathcal{E}}^{*}\right)$ is the incidence correspondence considered in $\S 1$. The inclusion in (2.7) gives rise to the morphism $p_{2}$ : $\mathbf{P}\left(\mathcal{N}_{\mathcal{E}}^{*}\right) \longrightarrow \mathbf{P}\left(H^{0}(\mathcal{E})\right)$. Then $U_{\mathcal{E}}$ and $\mathcal{Z}_{\mathcal{E}}=p_{2}^{-1}\left(U_{\mathcal{E}}\right)$ from $\S 1$ are Zariski open subsets in $\mathbf{P}\left(H^{0}(\mathcal{E})\right)$ and in $\mathbf{P}\left(\mathcal{N}_{\mathcal{E}}^{*}\right)$ respectively. In particular, $\mathcal{Z}_{\mathcal{E}}$ is a smooth, irreducible variety and we have the following diagram.

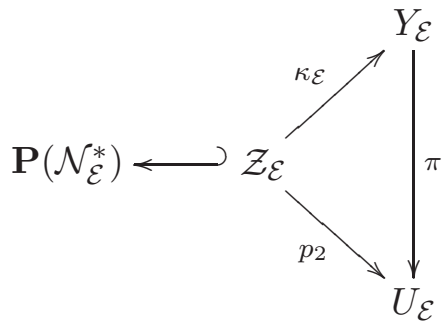

Setting $\mathcal{Z}^{\prime}$ to be the image of $\kappa_{\mathcal{E}}$ we obtain

$$
d=\operatorname{deg} \kappa_{\mathcal{E}} \cdot \operatorname{deg}\left(\left.\pi\right|_{\mathcal{Z}^{\prime}}\right) .
$$

By definition

$$
\kappa_{\mathcal{E}}^{*} \mathcal{O}_{Y_{\mathcal{E}}}(1)=p_{2}^{*} \mathcal{O}_{U_{\mathcal{E}}}(1)=\mathcal{O}_{\mathbf{P}\left(\mathcal{N}_{\mathcal{E}}^{*}\right)}(1) \otimes \mathcal{O}_{\mathcal{Z}_{\mathcal{E}}},
$$

where $\mathcal{O}_{\mathbf{P}\left(\mathcal{N}_{\mathcal{E}}^{*}\right)}(1)$ is such that $p_{1 *} \mathcal{O}_{\mathbf{P}\left(\mathcal{N}_{\mathcal{E}}^{*}\right)}(1)=\mathcal{N}_{\mathcal{E}}$. Since it is generated by global sections we have a morphism

$$
f_{\mathcal{E}}: \mathbf{P}\left(\mathcal{N}_{\mathcal{E}}^{*}\right) \longrightarrow \mathbf{P}\left(H^{0}\left(\mathcal{O}_{\mathbf{P}\left(\mathcal{N}_{\mathcal{E}}^{*}\right)}(1)\right)^{*}\right)=\mathbf{P}\left(H^{0}\left(\mathcal{N}_{\mathcal{E}}\right)^{*}\right)
$$

which factors through $Y_{\mathcal{E}}$. This implies that $\operatorname{deg} \kappa_{\mathcal{E}} \leqslant \operatorname{deg} f_{\mathcal{E}}$.

Remark 2.8. If $\delta_{\mathcal{E}} \geqslant 1$ and $\mathcal{N}_{\mathcal{E}}$ (the dual of $\mathcal{N}_{\mathcal{E}}^{*}$ in $(2.7)$ ) is very ample, then the cohomological invariant of $\mathcal{E}$ has degree 1 . In particular, $\mathcal{R}_{\mathcal{E}}$ determines $X$ up to birational isomorphism, unless $H^{0}\left(\mathcal{O}_{X}\left(K_{X}+L\right)\right)=0$. Indeed, the morphism $f_{\mathcal{E}}$ is an embedding. So $\kappa_{\mathcal{E}}: \mathcal{Z}_{\mathcal{E}} \longrightarrow \mathcal{Z}^{\prime}$ is an isomorphism. Applying Corollary 2.7 we obtain that $\mathcal{R}_{\mathcal{E}}$ determines $\mathcal{Z}_{\mathcal{E}}$ unless every point $p \in X$ is $L$-special. This can occur only if $H^{0}\left(\mathcal{O}_{X}\left(K_{X}+L\right)\right)=0$. Thus, if $H^{0}\left(\mathcal{O}_{X}\left(K_{X}+L\right)\right) \neq 0$, then $\mathcal{R}_{\mathcal{E}}$ determines $\mathcal{Z}_{\mathcal{E}}$. From the proof of Lemma 2.1 it also follows that we recover the finite morphism $p_{2}: \mathcal{Z}_{\mathcal{E}} \longrightarrow U_{\mathcal{E}}$. To determine $X$ (up to birational isomorphism) it is enough to observe that for a general plane $\mathbf{P}^{2} \subset \mathbf{P}\left(H^{0}(\mathcal{E})\right)$ the inverse image $p_{2}^{-1}\left(\mathbf{P}^{2}\right)$ is birational to $X$ (the projection $p_{1}: \mathbf{P}\left(\mathcal{N}_{\mathcal{E}}^{*}\right) \longrightarrow X$ restricted to $p_{2}^{-1}\left(\mathbf{P}^{2}\right)$ is the blowing-up of $X$ at $\left(L^{2}-d\right)$ points where the three-dimensional subspace of $H^{0}(\mathcal{E})$ defining $\mathbf{P}^{2}$ fails to generate $\left.\mathcal{E}\right)$.

A point of view complementary to the above considerations leads to a standard geometric realization of $\mathcal{E}$. Namely, we take the projectivization of $\mathcal{E}^{*}$ together with its natural projection $\sigma: \mathbf{P}_{\mathcal{E}}=\mathbf{P}\left(\mathcal{E}^{*}\right) \longrightarrow X$. Choose a tautological bundle $\mathcal{O}_{\mathbf{P}_{\mathcal{E}}}(1)$ so that $\sigma_{*} \mathcal{O}_{\mathbf{P}_{\mathcal{E}}}(1)=\mathcal{E}$ and consider the morphism $\phi_{\mathcal{E}}: \mathbf{P}_{\mathcal{E}} \longrightarrow \mathbf{P}\left(H^{0}(\mathcal{E})^{*}\right)$ defined by $\mathcal{O}_{\mathbf{P}_{\mathcal{E}}}(1)$. Then the standard geometric realization of $\mathcal{E}$ mentioned above is given as

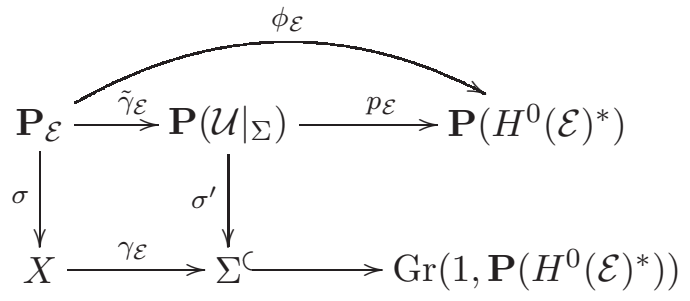




\section{INVARIANTS OF VECTOR BUNDLES}

where $\gamma_{\mathcal{E}}$ is the morphism defined by the linear subspace $i m\left(\wedge^{2} H^{0}(\mathcal{E}) \longrightarrow H^{0}\left(\mathcal{O}_{X}(L)\right)\right)$ of the space of the global sections of $\mathcal{O}_{X}(L), \Sigma$ is its image, and $\left.\mathcal{U}\right|_{\Sigma}$ is the restriction of the universal bundle of the Grassmannian $\operatorname{Gr}\left(1, \mathbf{P}\left(H^{0}(\mathcal{E})^{*}\right)\right.$ ). In particular, $\phi_{\mathcal{E}}=p_{\mathcal{E}} \circ \gamma_{\mathcal{E}}$ (if no ambiguity is likely we will omit the subscript $\mathcal{E}$ in the above notation).

TheOREm 2.9. Let $\mathcal{E}$ be a vector bundle of rank 2 over $X$. Assume that it is generated by global sections and its index of speciality $\delta_{\mathcal{E}} \geqslant 1$. If either $\mathcal{E}$ or $\mathcal{N}_{\mathcal{E}}$ (as in Remark 2.8) is very ample in the sense of Hartshorne, then the cohomological invariant of $\mathcal{E}$ has degree 1. In particular, $\mathcal{R}_{\mathcal{E}}$ determines $X$ up to a birational equivalence, unless $H^{0}\left(\mathcal{O}_{X}\left(K_{X}+L\right)\right)=0$.

Proof. In view of Remark 2.8 only the first part of the statement needs to be proved. Consider the diagram

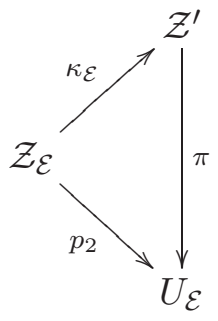

as in Proposition 2.3. We have $\operatorname{deg}\left(c_{2}(\mathcal{E})\right)=d=\operatorname{deg} p_{2}=\operatorname{deg} \kappa_{\mathcal{E}} \operatorname{deg} \pi$. First we show that the hypothesis that $\mathcal{E}$ is very ample implies that $\operatorname{deg} \kappa_{\mathcal{E}}=1$. Indeed, for $x \in X$ consider $P_{x}=\{e \in$ $\left.H^{0}(\mathcal{E}) \mid e(x)=0\right\}$, the space of sections of $\mathcal{E}$ vanishing at $x$. The fact that $\mathcal{E}$ is very ample implies that, for a general $x \in X$ and general choice $e, e^{\prime} \in P_{x}$, the curve $C=\left(e \wedge e^{\prime}=0\right)$ is irreducible and $x$ is the only common zero of $e$ and $e^{\prime}$. In particular, for any two distinct points $[s],[t]$ in the pencil $\left\{e, e^{\prime}\right\}$ spanned by $e$ and $e^{\prime}$ we have $Z_{s} \cap Z_{t}=\{x\}$, where $Z_{f}$ denotes the subscheme of zeros of a section $f$ of $\mathcal{E}$ and $[f]$ denotes the point of the pencil underlying the vector $f$ in the vector space spanned by sections $e$ and $e^{\prime}$.

Assume $\operatorname{deg} \kappa_{\mathcal{E}} \geqslant 2$. For every $[\alpha] \in P=\left\{e, e^{\prime}\right\} \cap U_{\mathcal{E}}$ we have a proper subscheme $Z_{\alpha, x}=$ $\kappa_{\mathcal{E}}^{*}\left(\kappa_{\mathcal{E}}((\alpha, x))\right)$ of $Z_{\alpha}$. From $\operatorname{deg} Z_{\alpha, x}=\operatorname{deg} \kappa_{\mathcal{E}} \geqslant 2$ it follows that $Z_{\alpha, x}$ moves on $C$ as $\alpha$ varies in the pencil (otherwise $Z_{e} \cap Z_{e^{\prime}} \supset Z_{\alpha, x}$ ). But then the $Z_{\alpha, x}$ trace out a component of $C$. Since $C$ is irreducible it follows that $C$ is the closure (in the complex topology) of $\bigcup_{\alpha \in P}\left(Z_{\alpha, x} \backslash\{x\}\right.$ ). Fix $e$ so that $Z_{e}$ is the set of $d$ distinct points and consider $y \in Z_{e} \backslash Z_{e, x}$. By the above considerations this point is the limit of a sequence of points $y_{n} \in Z_{e_{n}, x} \backslash\{x\}$, where $\left\{\left[e_{n}\right]\right\}$ is a sequence of points in $P$ converging to $[\beta] \in\left\{e, e^{\prime}\right\}$. It is clear that $[\beta] \neq[e]$. On the other hand $\beta(y)=\lim _{n \rightarrow \infty} e_{n}\left(y_{n}\right)=0$. So $y \in Z_{\beta} \cap\left(Z_{e} \backslash Z_{e, x}\right)$, contradicting the fact that $Z_{\beta} \cap Z_{e}=\{x\}$. Thus $\operatorname{deg} \kappa_{\mathcal{E}}=1$.

Applying Proposition 2.3 we obtain that $\mathcal{R}_{\mathcal{E}}$ determines $\mathcal{Z}_{\mathcal{E}}$ unless every point of $X$ is $L$-special. This occurs only if $H^{0}\left(\mathcal{O}_{X}\left(K_{X}+L\right)\right)=0$. The rest of the argument is as in Remark 2.8.

Corollary 2.10. Let $X$ be a nondegenerate surface in an Abelian variety $A$ and let $\Omega_{X}$ and $\mathcal{N}_{X / A}$ be respectively cotangent and normal bundles of $X$. Assume that either $\Omega_{X}$ or $\mathcal{N}_{X / A}$ is very ample. Then the cohomological invariant of $\Omega_{X}$ has degree 1 or $X$ is infinitesimally rigid in $A$. In particular, if $X$ has nontrivial deformations in $A$ (i.e. other than the ones coming from the group of translations in $A$ ), then $\mathcal{R}_{\Omega_{X}}$ determines $X$ up to an isomorphism.

Proof. Observe that $X$ is a minimal surface of general type. In particular, $H^{0}\left(\mathcal{O}_{X}\left(2 K_{X}\right)\right) \neq 0$. So if $\delta_{\Omega_{X}} \geqslant 1$ then Theorem 2.9 applied to $\Omega_{X}$ yields that $\mathcal{R}_{\Omega_{X}}$ has degree 1 and it determines $X$ up to birational equivalence. Once again using the fact that $X$ is minimal and of general type it follows that $X$ is determined up to an isomorphism. 


\section{REIDER}

Let $\delta_{\Omega_{X}}=0$ and consider the normal sequence of $X \subset A$,

$$
0 \longrightarrow \Theta_{X} \longrightarrow \Theta_{A} \otimes \mathcal{O}_{X} \longrightarrow \mathcal{N}_{X / A} \longrightarrow 0,
$$

where $\Theta_{X}$ (respectively $\Theta_{A}$ ) is the tangent bundle of $X$ (respectively, of $A$ ). From the cohomology sequence of $(2.10)$ we deduce that

$$
0=\delta_{\Omega_{X}} \geqslant \operatorname{dim}\left(\operatorname{ker}\left(H^{1}\left(\Theta_{X}\right) \longrightarrow H^{0}\left(\Theta_{A}\right) \otimes H^{1}\left(\mathcal{O}_{X}\right)\right)\right)=\operatorname{dim}\left(H^{0}\left(\mathcal{N}_{X / A}\right) / H^{0}\left(\Theta_{A}\right)\right),
$$

yielding the infinitesimal rigidity of $X$ in $A$.

Corollary 2.11. Let $X$ be a regular surface and let $\mathcal{E}$ be a very ample vector bundle of rank 2 over $X$. Let $\mathcal{O}_{X}(L)=\operatorname{det}(\mathcal{E})$ and assume that $H^{0}\left(\mathcal{O}_{X}\left(K_{X}+L\right) \neq 0\right.$. Then either $H^{1}\left(\mathcal{E}^{*}\right)=0$ or the cohomological invariant of $\mathcal{E}$ has degree 1 and it determines $X$ up to a birational equivalence.

Proof. From Remark 1.5, part c, it follows that $\delta_{\mathcal{E}}=h^{1}\left(\mathcal{E}^{*}\right)$. So if $h^{1}\left(\mathcal{E}^{*}\right) \geqslant 1$, Theorem 2.9 implies that the cohomological invariant of $\mathcal{E}$ has degree 1 and it determines $X$ up to birational equivalence.

\section{The cohomological invariant and geometry of $\phi_{\mathcal{E}}$}

In this section we will show that $\operatorname{deg} \mathcal{R}_{\mathcal{E}} \geqslant 2$ is akin to a 'speciality' of the vector bundle $\mathcal{E}$. In fact we have already seen in the proof of Theorem 2.9 that the condition $\operatorname{deg} \mathcal{R}_{\mathcal{E}} \geqslant 2$ forces the degree of $\phi_{\mathcal{E}}$ (see $(2.9)$ for notation) to be $\geqslant 2$. We will now consider this implication more closely.

Throughout this section the following conditions will be assumed:

1) $\mathcal{E}$ is generated by global sections and ample;

2) $\operatorname{deg} \gamma_{\mathcal{E}}=1$

3) $\operatorname{deg} \mathcal{R}_{\mathcal{E}} \geqslant 2$.

We already know that in this situation the degree of the morphism $p_{\mathcal{E}}$ in $(2.9)$ is $\geqslant 2$. We will analyze the configuration of lines in $\mathbf{P}\left(H^{0}(\mathcal{E})^{*}\right)$ corresponding to the zero-locus of a general global section of $\mathcal{E}$, and the aforementioned 'speciality' of $\mathcal{E}$ will be manifested in a speciality of this line configuration. For $x \in X$ we denote by $l_{x}$ the image of $\sigma^{-1}(x)$ under $\phi$. So the zero-locus $Z_{e}$ of a global section $e$ of $\mathcal{E}$ gives rise to the set of lines $\left\{l_{x}\right\}_{x \in Z_{e}}$ whose configuration we would like to understand.

We begin by defining families of curves on $X$ arising respectively from the incidence of lines in the image of $\phi$ (consequence of $\operatorname{deg} p_{\mathcal{E}} \geqslant 2$ ) and the hypothesis $\operatorname{deg} \mathcal{R}_{\mathcal{E}} \geqslant 2$.

Consider the incidence

$$
I=\overline{\left\{(x, y) \in X \times X \mid l_{x} \cap l_{y} \neq \emptyset, l_{x} \neq l_{y}\right\}} .
$$

Since $\operatorname{deg} p_{\mathcal{E}} \geqslant 2$ it follows that $I$ contains the divisorial part, which will be denoted by $I_{1}$. This induces a family of curves $\left\{B_{x}\right\}_{x \in X}$, where $B_{x}=\operatorname{pr}_{2 *}\left(\operatorname{pr}_{1}^{-1}(x) \cap I_{1}\right)\left(\mathrm{pr}_{i}, i=1,2\right.$, is the projection of $X \times X$ on the $i$ th factor).

If $\operatorname{deg} \mathcal{R}_{\mathcal{E}} \geqslant 2$ then we can define 'interesting' curves on $X$ as well. This is done as in the proof of Theorem 2.9: for a general $x \in X$ consider the subspace $P_{x}$ of global sections of $\mathcal{E}$ vanishing at $x$. The fact that $\operatorname{deg} \gamma_{\mathcal{E}}=1$ ensures that, for a general choice of two linearly independent sections $e$ and $e^{\prime}$ in $P_{x}$, the point $x$ is the only common zero of these two sections, i.e. $Z_{e} \cap Z_{e^{\prime}}=\{x\}$. Consider $\mathbf{P}^{1}$, the pencil spanned by $e$ and $e^{\prime}$. For every $[\alpha] \in P=\mathbf{P}^{1} \cap U_{\mathcal{E}}$ (see the proof of Theorem 2.9 for notation) we have a proper subscheme $Z_{\alpha, x}=\kappa_{\mathcal{E}}^{*}\left(\kappa_{\mathcal{E}}((\alpha, x))\right)$ of $Z_{\alpha}$. Arguing as in the proof of 


\section{INVARIANTS OF VECTOR BUNDLES}

Theorem 2.9 we see that $Z_{\alpha, x}$ moves as $\alpha$ varies in the pencil. This implies that

$$
\Gamma_{x}\left(e, e^{\prime}\right)=\overline{\bigcup_{[\alpha] \in P} Z_{\alpha, x} \backslash\{x\}}
$$

is a curve, a proper component of $C=\left(e \wedge e^{\prime}=0\right)$.

Remark 3.1. Using the notation of $(2.7)$ we see that the curves $\Gamma_{x}\left(e, e^{\prime}\right)$ are parametrized by a Zariski open subset in the relative Grassmannian of lines $\operatorname{Gr}\left(1, \mathbf{P}\left(\mathcal{N}_{\mathcal{E}}^{*}\right)\right)$.

The key point in understanding our line configuration will be a relationship between $B_{x}$ and $\Gamma_{x}\left(e, e^{\prime}\right)$. First observe that $B_{x}$ is the divisorial part of the base locus of the linear system corresponding to the subspace $V_{x}=i m\left(\wedge^{2} P_{x} \longrightarrow H^{0}\left(\mathcal{O}_{X}(L)\right)\right)$ of the sections of $\mathcal{O}_{X}(L)$. If $h^{0}(\mathcal{E}) \geqslant 5$ then $\operatorname{dim} V_{x} \geqslant 2$. So the linear system $\left|V_{x}\right|$ moves.

Lemma 3.2. Let $h^{0}(\mathcal{E}) \geqslant 5$. Then for general $x \in X$ the moving part of $\left|V_{x}\right|$ is not composed of a pencil.

Proof. The subspace $P_{x}$ defines a rational map

$$
\gamma_{P_{x}}: X-->\operatorname{Gr}\left(1, \mathbf{P}\left(P_{x}^{*}\right)\right)
$$

If the moving part of $\left|V_{x}\right|$ is composed of a pencil then the image of $\gamma_{P_{x}}$ is a curve. This implies that for any line $l_{y}$ which is skew to $l_{x}$ the $\mathbf{P}^{3}$ spanned by these two lines will contain a one-parameter family of lines $l_{z}$. But this is impossible since for general $x \in X$ and general $e \in P_{x}$ the hyperplane of $\mathbf{P}\left(H^{0}(\mathcal{E})^{*}\right)$ corresponding to $e$ contains no other lines $l_{z}$ but $\left\{l_{y}\right\}_{y \in Z_{e}}$ and for general $e$ there exists at least one $y \in Z_{e}$ such that $l_{y}$ is skew to $l_{x}$. Thus the image of $\gamma_{P_{x}}$ must be a surface.

For general $x \in X$ and $e, e^{\prime} \in P_{x}$ we have $C=\left(e \wedge e^{\prime}=0\right)=B_{x}+C\left(e, e^{\prime}\right)$, where $C\left(e, e^{\prime}\right)$ is the component of $C$ residual to $B_{x}$. From Lemma 3.2 it follows that for a general choice of $e, e^{\prime} \in P_{x}$ the curve $C\left(e, e^{\prime}\right)$ is reduced and irreducible. On the other hand

$$
B_{x}+C\left(e, e^{\prime}\right)=\Gamma_{x}\left(e, e^{\prime}\right)+\Gamma_{x},
$$

where $\Gamma_{x}$ is the component of $C$ residual to $\Gamma_{x}\left(e, e^{\prime}\right)$. Since $C\left(e, e^{\prime}\right)$ is irreducible it is a component of either $\Gamma_{x}\left(e, e^{\prime}\right)$ or $\Gamma_{x}$. This implies the following possibilities:

1) $\Gamma_{x} \subset B_{x}$

2) $\Gamma_{x}\left(e, e^{\prime}\right) \subset B_{x}$.

Remark 3.3.

a) $B_{x} \cdot L \leqslant \frac{1}{2} L^{2}$. This can be seen by taking $y \in B_{x}$ and considering the plane spanned by the lines $l_{x}$ and $l_{y}$. Then for a general choice of $\alpha, \beta \in H^{0}(\mathcal{E})$ whose corresponding hyperplanes in $\mathbf{P}\left(H^{0}(\mathcal{E})^{*}\right)$ contain the plane spanned by $l_{x}$ and $l_{y}$ we obtain

$$
C=(\alpha \wedge \beta=0) \supset B_{x}+B_{y},
$$

which implies $B_{x} \cdot L \leqslant \frac{1}{2} L^{2}$.

b) If the image of $\phi$ has degree $\geqslant 3$ then

$$
B_{x} \cdot L<\frac{1}{2} L^{2} .
$$

Indeed, the intersection of the image of $\phi$ with the codimension- 2 subspace $H_{\alpha} \cap H_{\beta}$ is a subscheme of degree $\geqslant 3\left(H_{s}\right.$ denotes the hyperplane corresponding to $s \in H^{0}(\mathcal{E})$ via the 


\section{REIDER}

natural identification $\left.H^{0}\left(\mathcal{O}_{\mathbf{P}\left(H^{0}(\mathcal{E})^{*}\right)}(1)\right)=H^{0}(\mathcal{E})\right)$. This implies that $B_{x}+B_{y}$ in $(3.3)$ is a proper component of $C$.

From the inequality (3.4) it follows that if possibility 1 (respectively 2 ) in (3.2) holds for some $x \in X$ and $e, e^{\prime} \in P_{x}$, then the same inclusion holds for all $x \in X$ subject to Lemma 3.2 and $e, e^{\prime} \in P_{x}$ for which $\Gamma_{x}$ (respectively $\Gamma_{x}\left(e, e^{\prime}\right)$ ) is defined. Indeed, possibility 1 implies $\Gamma_{x} . L \leqslant B_{x} . L<\frac{1}{2} L^{2}$. This yields $\Gamma_{x}(\alpha, \beta) . L>\frac{1}{2} L^{2}$ for a general choice of $\alpha, \beta \in P_{x}$. For any other $x^{\prime} \in X$ subject to Lemma 3.2 and general choice of $\alpha^{\prime}, \beta^{\prime} \in P_{x^{\prime}}$ we have

$$
\Gamma_{x^{\prime}}+\Gamma_{x^{\prime}}\left(\alpha^{\prime}, \beta^{\prime}\right)=B_{x^{\prime}}+C\left(\alpha^{\prime}, \beta^{\prime}\right),
$$

where $C\left(\alpha^{\prime}, \beta^{\prime}\right)$ is the component of $C=\left(\alpha^{\prime} \wedge \beta^{\prime}=0\right)$ residual to $B_{x^{\prime}}$ and $C\left(\alpha^{\prime}, \beta^{\prime}\right)$ is reduced and irreducible. This together with $\Gamma_{x^{\prime}}\left(\alpha^{\prime}, \beta^{\prime}\right) \cdot L=\Gamma_{x}(\alpha, \beta) . L>\frac{1}{2} L^{2}$ imply that $C\left(\alpha^{\prime}, \beta^{\prime}\right)$ must be a component of $\Gamma_{x^{\prime}}\left(\alpha^{\prime}, \beta^{\prime}\right)$ which gives the inclusion $\Gamma_{x^{\prime}} \subset B_{x^{\prime}}$.

Lemma 3.4. If $h^{0}(\mathcal{E}) \geqslant 6$, then for general $x \in X$ and general choice of $e, e^{\prime} \in P_{x}$ one has the inclusion $\Gamma_{x}\left(e, e^{\prime}\right) \subset B_{x}$.

Proof. Assume that $\Gamma_{x}\left(e, e^{\prime}\right) \not \subset B_{x}$. Then we must have $\Gamma_{x} \subset B_{x}$.

Claim 3.5. $Z_{e} \backslash Z_{e, x}$ is contained in $\Gamma_{x}$.

Let us assume this claim and complete the proof of the lemma. From the claim and the inclusion $\Gamma_{x} \subset B_{x}$ it follows that $l_{y} \cap l_{x} \neq \emptyset$, for every $y \in Z_{e} \backslash Z_{e, x}$. By Remark 3.3, part b, the inclusion $\Gamma_{x^{\prime}} \subset B_{x^{\prime}}$ holds for any $x^{\prime} \in Z_{e, x}$. Using Claim 3.5 for $x^{\prime}$ we deduce $Z_{e} \backslash Z_{e, x^{\prime}} \subset \Gamma_{x^{\prime}}$ for every $x^{\prime} \in Z_{e, x}$. But $Z_{e} \backslash Z_{e, x^{\prime}}=Z_{e} \backslash Z_{e, x}$ for every $x^{\prime} \in Z_{e, x}$. Thus we obtain

$$
l_{y} \cap l_{x^{\prime}} \neq \emptyset \forall y \in Z_{e} \backslash Z_{e, x} \text { and } \forall x^{\prime} \in Z_{e, x} .
$$

We may assume that $e$ is chosen so that there is $y \in Z_{e, x}$ such that $l_{y} \cap l_{x}=\emptyset$ (otherwise $\left.\Gamma_{x}\left(e, e^{\prime}\right) \subset B_{x}\right)$. Since the lines corresponding to the points in $Z_{e} \backslash Z_{e, x}$ intersect both $l_{x}$ and $l_{y}$ it follows that they must be contained in $\mathbf{P}^{3}$ spanned by $l_{x}$ and $l_{y}$. But the remaining lines $l_{x^{\prime}}$ with $x^{\prime} \in Z_{e, x}$ also intersect all the lines $l_{z}$ with $z \in Z_{e} \backslash Z_{e, x}$. This implies that either all the $l_{z}$ with $z \in Z_{e} \backslash Z_{e, x}$ pass through a common point or the set of lines $\left\{l_{z}\right\}_{z \in Z_{e}}$ is contained in $\mathbf{P}^{3}$. But the latter contradicts the assumption $h^{0}(\mathcal{E}) \geqslant 6$ (the lines $\left\{l_{z}\right\}_{z \in Z_{e}}$ span the hyperplane in $\mathbf{P}\left(H^{0}(\mathcal{E})^{*}\right)$ corresponding to $e$ so their span has dimension $\left.h^{0}(\mathcal{E})-2 \geqslant 4\right)$. Thus we obtain that all lines $l_{z}$ with $z \in Z_{e} \backslash Z_{e, x}$ have a point in common. Call this point $a(e, x)$. We have seen that $a(e, x) \notin l_{x}$ and all other lines $l_{x^{\prime}}$ with $x^{\prime} \in Z_{e, x}$ either pass through $a(e, x)$ or are contained in the plane spanned by $l_{x}$ and $a(e, x)$.

The point $a(e, x)$ distinguishes in $\left\{l_{x^{\prime}}\right\}_{x^{\prime}} \in Z_{e, x}$ the proper subset of lines passing through $a(e, x)$. This gives rise to a proper subcycle $Z_{e, x}^{\prime}$ of $Z_{e, x}$. We will now consider what happens as we move $e$ in a general pencil $\left\{e, e^{\prime}\right\}$ in $P_{x}$.

First observe that the point $a(e, x)$ must move as we vary $e$ (otherwise we have a curve in $\mathbf{P}_{\mathcal{E}}$ contracted to $a(e, x)$ by $\phi$, which contradicts the ampleness of $\mathcal{E})$. This implies that as $[\alpha]$ moves in the pencil $\left\{e, e^{\prime}\right\}$ the points $a(\alpha, x)$ trace out a curve which we denote by $D_{x}\left(e, e^{\prime}\right)$. Hence the subcycles $Z_{\alpha, x}^{\prime}$ will trace out a component $\Gamma_{x}^{\prime}\left(e, e^{\prime}\right)$ of $\Gamma_{x}\left(e, e^{\prime}\right)$. Then

$$
\Gamma_{x}\left(e, e^{\prime}\right)=\Gamma_{x}^{\prime}\left(e, e^{\prime}\right)+\Gamma_{x}^{\prime \prime}\left(e, e^{\prime}\right),
$$

where $\Gamma_{x}^{\prime \prime}\left(e, e^{\prime}\right)$ is the component of $\Gamma_{x}\left(e, e^{\prime}\right)$ residual to $\Gamma_{x}^{\prime}\left(e, e^{\prime}\right)$.

The curve $D_{x}\left(e, e^{\prime}\right)$ gives rise to a one-parameter family of planes passing through $l_{x}$. As we move our pencil $\left\{e, e^{\prime}\right\}$ in the Grassmannian of lines of $\mathbf{P}\left(P_{x}\right)$, the curve $D_{x}\left(e, e^{\prime}\right)$ cannot move (otherwise we would have a two-parameter family of planes passing through $l_{x}$ giving rise to a two-parameter family of fibres of $\mathbf{P}_{\mathcal{E}}$ whose image under $\phi$ intersects $l_{x}$; this again contradicts the ampleness of $\mathcal{E}$ ). This implies that the component $\Gamma_{x}^{\prime \prime}\left(e, e^{\prime}\right)$ in (3.6) does not move as we vary the pencil $\left\{e, e^{\prime}\right\}$. 


\section{INVARIANTS OF VECTOR BUNDLES}

Since $\Gamma_{x}\left(e, e^{\prime}\right)$ moves, it follows that $\Gamma_{x}^{\prime}\left(e, e^{\prime}\right)$ must move. But this implies that, through any point of $D_{x}\left(e, e^{\prime}\right)$, there passes a one-parameter family of line images of the fibres of $\mathbf{P}_{\mathcal{E}}$. Hence there are (infinitely many) curves in $\mathbf{P}_{\mathcal{E}}$ contracted by $\phi$. Again this is a contradiction to the ampleness of $\mathcal{E}$.

This completes the proof of Lemma 3.4.

We now return to Claim 3.5.

Proof of Claim 3.5. Let there be $y \in Z_{e} \backslash Z_{e, x}$ such that $y \in \Gamma_{x}\left(e, e^{\prime}\right)$. Let $\mathbf{P}^{1}$ be a general pencil $\left\{e, e^{\prime}\right\}$ chosen so that $Z_{e} \cap Z_{e^{\prime}}=\{x\}$ and let $U_{\mathcal{E}}$ be as in the proof of Theorem 2.9. Recall that

$$
\Gamma_{x}\left(e, e^{\prime}\right)=\overline{\bigcup_{[\alpha] \in P} Z_{\alpha, x} \backslash\{x\}},
$$

where $P=\mathbf{P}^{1} \cap U_{\mathcal{E}}$. Hence there exists a sequence of $y_{n} \in Z_{\alpha_{n}, x} \backslash\{x\},\left[\alpha_{n}\right] \in P$, such that $y=\lim _{n} y_{n}$. Let $[\alpha]=\lim _{n}\left[\alpha_{n}\right]$. It is clear that $[\alpha] \neq[e]$ and $\alpha(y)=\lim _{n} \alpha_{n}\left(y_{n}\right)=0$. So $y \in Z_{\alpha} \cap\left(Z_{e} \backslash Z_{e, x}\right)$. Hence $Z_{\alpha} \cap Z_{e}=Z_{e^{\prime}} \cap Z_{e} \supset\{x, y\}$, contradicting the choice of the pencil $\left\{e, e^{\prime}\right\}$.

Remark 3.6. If $h^{0}(\mathcal{E})=5$ and $\operatorname{deg}(i m(\phi)) \geqslant 3$ and if $\Gamma_{x}\left(e, e^{\prime}\right) \not \subset B_{x}$ for general $x \in X$ and general $e, e^{\prime} \in P_{x}$, then the proof of Lemma 3.4 shows that the set of lines $\left\{l_{z}\right\}_{z \in Z_{e}}$ is contained in a smooth quadric $Q$ in $\mathbf{P}^{3}$ (the hyperplane of $\mathbf{P}^{4}=\mathbf{P}\left(H^{0}(\mathcal{E})^{*}\right)$ corresponding to $e$ ). Furthermore, the lines $\left\{l_{x^{\prime}}\right\}_{x^{\prime}} \in Z_{e, x}$ and $\left\{l_{z}\right\}_{z \in Z_{e} \backslash Z_{e, x}}$ belong to two distinct families of rulings of $Q=\mathbf{P}^{1} \times \mathbf{P}^{1}$.

We will now use Lemma 3.4 to analyze the configuration of lines $\left\{l_{x}\right\}_{x \in Z_{e}}$ for a general global section $e$ of $\mathcal{E}$.

Proposition 3.7. Let

$$
Z_{e}=\sum_{p \in \pi^{-1}([e])} \kappa_{\mathcal{E}}^{*}(p)
$$

be the decomposition (provided by Corollary 2.7) of the zero-locus $Z_{e}=(e=0)$ for general $e \in$ $H^{0}(\mathcal{E})$. If $h^{0}(\mathcal{E}) \geqslant 6$, then the lines $\left\{l_{x}\right\}_{x \in \kappa_{\mathcal{E}}^{*}(p)}$ span a plane for every $p \in \pi^{-1}([e])$.

Proof. For $x \in \kappa_{\mathcal{E}}^{*}(p)$ consider a general pencil $\left\{e, e^{\prime}\right\}$ of sections vanishing at $x$. From Lemma 3.4 it follows that $\Gamma_{x}\left(e, e^{\prime}\right) \subset B_{x}$ which implies $l_{y} \cap l_{x} \neq \emptyset$ for all $y \in \kappa_{\mathcal{E}}^{*}(p) \backslash\{x\}$. Since this holds for any $x \in \kappa_{\mathcal{E}}^{*}(p)$ we obtain

$$
l_{y} \cap l_{x} \neq \emptyset \forall x \neq y \in \kappa_{\mathcal{E}}^{*}(p) .
$$

This implies that either the line configuration $\left\{l_{z}\right\}_{z \in \kappa_{\mathcal{E}}^{*}(p)}$ is as asserted in the proposition or all lines corresponding to the points in $\kappa_{\mathcal{E}}^{*}(p)$ pass through the same point, say $a(e, p)$, and span a projective subspace of dimension $\geqslant 3$. But the latter is impossible for general $e \in H^{0}(\mathcal{E})$. Indeed, if the dimension of the span of lines $\left\{l_{z}\right\}_{z \in \kappa_{\mathcal{E}}^{*}(p)}$ is $>3$, then the point $a(e, p)$ is a singular point of the image of $\phi$. If we move $e$ in $P_{x}$ the point $a(e, p)$ must move along $l_{x}$ since otherwise $\mathcal{E}$ fails to be ample. This implies that $l_{x}$ lies in the singular locus of the image of $\phi$. But there is at most a one-dimensional family of such points $x \in X$. Hence $e$ can be chosen so that its zero-locus $Z_{e}$ contains none of these points. Thus we may assume that the dimension of the $\operatorname{span}\left\{l_{z}\right\}_{z \in \kappa_{\varepsilon}^{*}(p)}$ is 3 and the point $a(e, p)$ is a smooth point of $i m(\phi)$. In this case the section $e$ corresponds to the hyperplane $H_{e}$ in $\mathbf{P}\left(H^{0}(\mathcal{E})^{*}\right)$ containing the embedded tangent space to $i m(\phi)$ at the point $a(e, p)$. But the variety of such hyperplanes is the tangent variety of $i m(\phi)$ which has codimension $\geqslant 1$ in $\mathbf{P}\left(H^{0}(\mathcal{E})\right)$. Thus a general choice of $e$ does not correspond to a tangent hyperplane.

This proposition will eventually serve us to show that the cohomological invariant determines the variety $\mathcal{Z}^{\prime}$ of Proposition 2.3 unless $\mathcal{E}$ is subject to some restrictions (see Theorem 3.11). But first we will need to establish some properties of the family $\left\{B_{x}\right\}_{x \in X}$. 


\section{REIDER}

Proposition 3.8. Let $B$ be the numerical equivalence class of the family $\left\{B_{x}\right\}_{x \in X}$. If $h^{0}(\mathcal{E}) \geqslant 6$ and $\operatorname{deg}\left(\mathcal{R}_{\mathcal{E}}\right) \geqslant 2$, then the following inequalities hold:

i) $\left(\left[\left(h^{0}(\mathcal{E})-2\right) / 3\right] \operatorname{deg}\left(\mathcal{R}_{\mathcal{E}}\right)\right) B \cdot L \leqslant L^{2}$, where $[\cdot]$ denotes the integral part of a real number;

ii) $\max \left(\operatorname{deg}\left(\phi_{\mathcal{E}}\right), \operatorname{deg}\left(\mathcal{R}_{\mathcal{E}}\right)\right) \leqslant B^{2}+2$.

Proof. Choose a general section $e$ of $\mathcal{E}$ and let $H_{e}$ be the hyperplane in $\mathbf{P}\left(H^{0}(\mathcal{E})^{*}\right)$ corresponding to $e$. This hyperplane is spanned by the lines $\left\{l_{z}\right\}_{z \in Z_{e}}$. Let

$$
Z_{e}=\sum_{p \in \pi^{-1}([e])} \kappa_{\mathcal{E}}^{*}(p)
$$

be the decomposition of $Z_{e}$ as in Proposition 3.7 and let $\Pi_{p}$ be the plane spanned by the lines $\left\{l_{x}\right\}_{x \in \kappa_{\mathcal{E}}^{*}(p)}$. This gives us a configuration of planes $\left\{\Pi_{p}\right\}_{p \in \pi^{-1}([e])}$.

A choice of a pencil $\left\{e, e^{\prime}\right\}$ of sections of $\mathcal{E}$ defines a curve $C=\left(e \wedge e^{\prime}=0\right)$ in the linear system $|L|$. We can choose $e^{\prime}$ so that the codimension-2 subspace $H_{e} \cap H_{e^{\prime}}$ contains at least $n=\left[\left(h^{0}(\mathcal{E})-2\right) / 3\right]$ planes of the configuration $\left\{\Pi_{p}\right\}_{p \in \pi^{-1}([e])}$, say $\Pi_{p_{1}}, \ldots, \Pi_{p_{n}}$. Each plane $\Pi_{p_{i}}$ gives rise to a component $C_{i}=\sum_{x \in \kappa_{\mathcal{E}}^{*}\left(p_{i}\right)} B_{x}$ of $C$ which implies $C \supset \sum_{i=1}^{n} C_{i}$. From this we obtain

$$
\left(n \operatorname{deg}\left(\mathcal{R}_{\mathcal{E}}\right)\right) B \cdot L=\left(\left[\frac{h^{0}(\mathcal{E})-2}{3}\right] \operatorname{deg}\left(\mathcal{R}_{\mathcal{E}}\right)\right) B \cdot L \leqslant L^{2},
$$

the first inequality of the proposition. To prove the second assertion we consider $x \neq y \in \kappa_{\mathcal{E}}^{*}(p)$. Then it is clear that $B^{2}=B_{x} \cdot B_{y} \geqslant \operatorname{deg}\left(\mathcal{R}_{\mathcal{E}}\right)-2$. Furthermore, let $a=l_{x} \cap l_{y}$ and let $m_{a}$ be the number of lines in $\left\{l_{z}\right\}_{z \in \kappa_{\mathcal{E}}^{*}(p)}$ passing through $a$. Then

$$
B^{2}=B_{x} \cdot B_{y} \geqslant \operatorname{deg}\left(\phi_{\mathcal{E}}\right)-m_{a}+\operatorname{deg}\left(\mathcal{R}_{\mathcal{E}}\right)-2,
$$

which combined with $m_{a} \leqslant \operatorname{deg}\left(\mathcal{R}_{\mathcal{E}}\right)$ yields the second inequality of the proposition.

Proposition 3.9. The divisor $B$ is nef. Furthermore, $B^{2}>0$ unless $\operatorname{deg}\left(\mathcal{R}_{\mathcal{E}}\right)=\operatorname{deg}\left(\phi_{\mathcal{E}}\right)=2$ and $\left\{B_{x}\right\}_{x \in X}$ is a family of rational curves.

Proof. Let $\Gamma$ be a curve on $X$ such that $\Gamma . B<0$. But then $\Gamma$ must be a common component of all the $B_{x}$, which is obviously impossible. Hence $B$ is nef.

If $B^{2}=0$ then Proposition 3.8, part ii, and its proof imply $\operatorname{deg}\left(\mathcal{R}_{\mathcal{E}}\right)=\operatorname{deg}\left(\phi_{\mathcal{E}}\right)=2$ and $x \notin B_{x}$, for general $x \in X$. To see the last assertion we consider $\mathbf{P}\left(\mathcal{E}^{*} \otimes \mathcal{O}_{B_{x}}\right)$. It contains a section denoted $S_{x}$ such that $\phi_{\mathcal{E}}\left(S_{x}\right)=l_{x}$. Thus the curve $B_{x}$ comes equipped with a surjective morphism $f_{x}: B_{x} \longrightarrow \mathbf{P}^{1}=l_{x}$. Observe that in the case at hand $\operatorname{deg}\left(f_{x}\right)=\operatorname{deg}\left(\phi_{\mathcal{E}}\right)-1=1$. Hence $B_{x}$ is rational.

Corollary 3.10. If $B^{2} \geqslant 1$ then

$$
\operatorname{deg}\left(\mathcal{R}_{\mathcal{E}}\right) \cdot\left[\frac{h^{0}(\mathcal{E})-2}{3}\right] \leqslant \sqrt{\frac{L^{2}}{B^{2}}}
$$

Proof. Use the choice of $C \in|L|$ as in the proof of Proposition 3.8 and the fact that $B$ is nef (Proposition 3.9) to deduce

$$
B . L \geqslant\left(n \operatorname{deg}\left(\mathcal{R}_{\mathcal{E}}\right)\right) B^{2},
$$

where $n=\left[\left(h^{0}(\mathcal{E})-2\right) / 3\right]$. This and the first inequality of Proposition 3.8 imply

$$
\left(n \operatorname{deg}\left(\mathcal{R}_{\mathcal{E}}\right)\right)^{2} B^{2} \leqslant L^{2},
$$

which gives the asserted inequality. 


\section{INVARIANTS OF VECTOR BUNDLES}

Theorem 3.11. Let $\mathcal{E}$ be subject to the conditions in $(3.1)$ and let $h^{0}(\mathcal{E}) \geqslant 6$. Then the cohomological invariant of $\mathcal{E}$ determines the variety $\mathcal{Z}^{\prime}$ in Proposition 2.3 unless one of the following occurs:

1) $h^{0}(\mathcal{E})=6, L^{2}=12, \operatorname{deg}\left(c_{2}(\mathcal{E})\right)=6, B^{2}=1, \operatorname{deg}\left(\mathcal{R}_{\mathcal{E}}\right)=\operatorname{deg}\left(\phi_{\mathcal{E}}\right)+1=3$;

2) $h^{0}(\mathcal{E}) \leqslant 7,13 \leqslant L^{2} \leqslant 16, B^{2}=1, \operatorname{deg}\left(\mathcal{R}_{\mathcal{E}}\right)=3$.

Proof. From Corollary 2.7 we know that $\mathcal{R}_{\mathcal{E}}$ fails to recover $\mathcal{Z}^{\prime}$ because the fibres of $\kappa_{\mathcal{E}}: \mathcal{Z}_{\mathcal{E}} \longrightarrow \mathcal{Z}^{\prime}$ are $L$-special, i.e. in the decomposition

$$
Z_{e}=\sum_{p \in \pi^{-1}([e])} \kappa_{\mathcal{E}}^{*}(p)
$$

the subcycles $\kappa_{\mathcal{E}}^{*}(p)$ are $L$-special with index of $L$-speciality $\delta\left(L, \kappa_{\mathcal{E}}^{*}(p)\right) \stackrel{\text { def }}{=} \operatorname{dim} H^{1}\left(\mathcal{I}_{\kappa_{\mathcal{E}}^{*}(p)}\left(K_{X}+L\right)\right)=$ 1. The argument is based on the following simple idea: If $\kappa_{\mathcal{E}}^{*}(p)$ is $L$-special then try to find a curve, say $E_{p}$, passing through $\kappa_{\mathcal{E}}^{*}(p)$ such that the restriction of $\mathcal{E}$ to $E_{p}$ fails to be ample.

This can be realized as follows. Use Serre duality

$$
\left(H^{1}\left(\mathcal{I}_{\kappa_{\mathcal{E}}^{*}(p)}\left(K_{X}+L\right)\right)\right)^{*}=\operatorname{Ext}^{1}\left(\mathcal{I}_{\kappa_{\mathcal{E}}^{*}(p)}(L), \mathcal{O}_{X}\right)
$$

to deduce that $\kappa_{\mathcal{E}}^{*}(p)$ gives rise to the sheaf $\mathcal{E}_{p}$ defined by the following exact sequence:

$$
0 \longrightarrow \mathcal{O}_{X} \longrightarrow \mathcal{E}_{p} \longrightarrow \mathcal{I}_{\kappa_{\mathcal{E}}^{*}(p)}(L) \longrightarrow 0
$$

The fact that $\delta\left(L, \kappa_{\mathcal{E}}^{*}(p)\right)=1$ implies that $\mathcal{E}_{p}$ is locally free. The Chern numbers of $\mathcal{E}_{p}$ are $c_{1}^{2}\left(\mathcal{E}_{p}\right)=$ $L^{2}$ and $\operatorname{deg}\left(c_{2}\left(\mathcal{E}_{p}\right)\right)=\operatorname{deg}\left(\kappa_{\mathcal{E}}^{*}(p)\right)=\operatorname{deg}\left(\mathcal{R}_{\mathcal{E}}\right)$. It is easy to see that the Bogomolov inequality $L^{2} \leqslant 4 \operatorname{deg}\left(\mathcal{R}_{\mathcal{E}}\right)$ can hold only if we are in the situation 1 of the theorem. So from now on we assume that $L^{2}>4 \operatorname{deg}\left(\mathcal{R}_{\mathcal{E}}\right)$ and use the Bogomolov destabilizing exact sequence

$$
0 \longrightarrow \mathcal{O}_{X}\left(D_{p}\right) \longrightarrow \mathcal{E}_{p} \longrightarrow \mathcal{I}_{A}\left(L-D_{p}\right) \longrightarrow 0,
$$

where $\mathcal{I}_{A}$ is the sheaf of ideals of some zero-dimensional subscheme $A$ and the divisor $D_{p}$ is such that $\left(2 D_{p}-L\right)$ lies in the positive cone of the Néron-Severi group $N(X)$ of $X$ (see [Rei77]). This, combined with the defining sequence of $\mathcal{E}_{p}$ in (3.7), gives a distinguished divisor denoted $E_{p}$ in $\left|L-D_{p}\right|$ such that $H^{0}\left(\mathcal{I}_{\kappa_{\mathcal{E}}^{*}(p)}\left(E_{p}\right)\right) \neq 0$, i.e. $E_{p}$ passes through $\kappa_{\mathcal{E}}^{*}(p)$. On the other hand, for general $e, e^{\prime} \in H^{0}(\mathcal{E})$, the curve $C=\left(e \wedge e^{\prime}=0\right)$ is reduced and irreducible and passes through $Z_{e}$. Hence the complete intersection $D$ of the curves $C$ and $E_{p}$ is zero-dimensional and $D \supset \kappa_{\mathcal{E}}^{*}(p)$.

Claim 3.12. $D=\kappa_{\mathcal{E}}^{*}(p)$ unless the situation 2 of Theorem 3.11 holds.

Let us assume this claim and show that the equality of the claim leads to the failure of $\mathcal{E}$ to be ample. Indeed, $D=\kappa_{\mathcal{E}}^{*}(p)$ implies that the section $e$ restricted to $E_{p}$ vanishes precisely on the divisor $\kappa_{\mathcal{E}}^{*}(p)$ in the linear system $|L|_{E_{p}} \mid$; in other words $\mathcal{E} \otimes \mathcal{O}_{E_{p}}(-L)$ has a nowhere vanishing section. This gives the exact sequence

$$
0 \longrightarrow \mathcal{O}_{E_{p}}(L) \longrightarrow \mathcal{E}_{p} \otimes \mathcal{O}_{E_{p}} \longrightarrow \mathcal{O}_{E_{p}} \longrightarrow 0
$$

which contradicts the fact that $\mathcal{E}$ is ample.

Thus the proof of Theorem 3.11 will be completed once we prove Claim 3.12.

Proof of Claim 3.12. Since we already know that $D \supset \kappa_{\mathcal{E}}^{*}(p)$ it will be enough to show that $\operatorname{deg} D=$ $\operatorname{deg}\left(\kappa_{\mathcal{E}}^{*}(p)\right)$, or, equivalently, L. $E_{p}=\operatorname{deg}\left(c_{2}\left(\mathcal{E}_{p}\right)\right)$. Computing the second Chern class from (3.8) we obtain

$$
\operatorname{deg}\left(\mathcal{R}_{\mathcal{E}}\right)=\operatorname{deg}\left(c_{2}\left(\mathcal{E}_{p}\right)\right)=D_{p} \cdot E_{p}+\operatorname{deg}(A) \geqslant L \cdot E_{p}-E_{p}^{2}
$$




\section{REIDER}

Thus the claim follows if we have $E_{p}^{2}=0$. This is indeed so unless the condition 2 occurs. To verify this, consider the cases according the values of $B^{2}$.

Case a) $B^{2}=0$. From Proposition 3.8, part ii, it follows that $\operatorname{deg}\left(\mathcal{R}_{\mathcal{E}}\right)=2$. Substituting this in $(3.9)$ we obtain

$$
\text { L. } E_{p}-E_{p}^{2} \leqslant 2 .
$$

From the Hodge index and $L . E_{p}<\frac{1}{2} L^{2}$ it follows that

$$
E_{p}^{2}<\frac{1}{2}\left(L \cdot E_{p}\right)
$$

which combined with (3.10) yields $L . E_{p} \leqslant 3$. Using the Hodge index once again we obtain $\left(L^{2}\right)\left(E_{p}^{2}\right) \leqslant 9$. But it is easy to see that $L^{2}$ must be at least 10 . Hence $E_{p}^{2}=0$.

Case b) $B^{2} \geqslant 1$. From case a it follows that we may also assume that $\operatorname{deg}\left(\mathcal{R}_{\mathcal{E}}\right) \geqslant 3$. In particular, $L^{2}>4 \operatorname{deg}\left(\mathcal{R}_{\mathcal{E}}\right)=12$, yielding the lower bound on $L^{2}$ in condition 2 . To obtain the upper bound on $L^{2}$ as well as the rest of the condition 2 we use (3.9) and (3.11) to deduce

$$
\operatorname{deg}\left(\mathcal{R}_{\mathcal{E}}\right)>\frac{1}{2}\left(L \cdot E_{p}\right) .
$$

This combined with Corollary 3.10 yields

$$
L . E_{p}<\frac{2}{n} \sqrt{\frac{L^{2}}{B^{2}}}
$$

where $n=\left[\left(h^{0}(\mathcal{E})-2\right) / 3\right]$. Using the Hodge index once again gives

$$
\left(E_{p}^{2}\right)\left(L^{2}\right)<\frac{4}{n^{2}} \frac{L^{2}}{B^{2}} \Longleftrightarrow E_{p}^{2}<\frac{4}{n^{2} B^{2}} .
$$

Thus $E_{p}^{2}=0$, unless $n=1$ and $B^{2} \leqslant 3$. Combining this with Proposition 3.8, part ii, we obtain $\operatorname{deg}\left(\mathcal{R}_{\mathcal{E}}\right) \leqslant 5$.

From now on we assume $E_{p}^{2} \geqslant 1$ and show that $B^{2}=1$. Indeed, if $B^{2} \geqslant 2$ then by (3.12) we have $E_{p}^{2}=1$. Substituting this in $(3.9)$ and using $\operatorname{deg}\left(\mathcal{R}_{\mathcal{E}}\right) \leqslant 5$ we obtain $L . E_{p} \leqslant 6$. From the Hodge index it follows that $L^{2} \leqslant 36$. Substituting this in the inequality of Corollary 3.10 we improve the bound on the degree of $\mathcal{R}_{\mathcal{E}}$, i.e. $\operatorname{deg}\left(\mathcal{R}_{\mathcal{E}}\right) \leqslant 4$. Repeating the above argument we obtain an improvement L. $E_{p} \leqslant 5$, yielding $L^{2} \leqslant 25$. Using Corollary 3.10 once again we obtain $\operatorname{deg}\left(\mathcal{R}_{\mathcal{E}}\right) \leqslant 3$, which leads, with the above reasoning repeated, to $L^{2} \leqslant 16$. But then by Corollary 3.10 we have $\operatorname{deg}\left(\mathcal{R}_{\mathcal{E}}\right) \leqslant 2$, which is contrary to our assumption. Thus we must have $B^{2}=1$. From Proposition 3.8, part ii, and the assumption $\operatorname{deg}\left(\mathcal{R}_{\mathcal{E}}\right) \geqslant 3$, it follows that $\operatorname{deg}\left(\mathcal{R}_{\mathcal{E}}\right)=3$. Substituting this in (3.9) we obtain L. $E_{p}-E_{p}^{2} \leqslant 3$. This and the Hodge index yield

$$
\left(E_{p}^{2}\right)\left(L^{2}\right) \leqslant\left(E_{p}^{2}+3\right)^{2} .
$$

Solving for $E_{p}^{2}$ gives

$$
E_{p}^{2} \leqslant \frac{18}{L^{2}-6+\sqrt{L^{2}\left(L^{2}-12\right)}} .
$$

From this it follows that $L^{2} \leqslant 16$.

We will now apply the above theorem to $\mathcal{E}=\Omega_{X}$, the cotangent bundle of $X$. In this case the morphism $\gamma_{\Omega_{X}}$ in (2.9) is classically known as the Gauss map.

Corollary 3.13. Let $X$ be a smooth projective surface with $\Omega_{X}$ generated by global sections and ample, and let $\delta_{\Omega_{X}} \geqslant 1$. Assume the Gauss map of $X$ to be of degree 1 onto its image 


\section{INVARIANTS OF VECTOR BUNDLES}

and $q_{X}=h^{0}\left(\Omega_{X}\right) \geqslant 6$. Then the cohomological invariant of $\Omega_{X}$ determines the variety $\mathcal{Z}^{\prime}$ in Proposition 2.3. Furthermore, if $\operatorname{deg}\left(\mathcal{R}_{\Omega_{X}}\right) \geqslant 2$, then

$$
\left[\frac{q_{X}-2}{3}\right] \leqslant \frac{1}{\operatorname{deg}(\mathcal{R})} \sqrt{\frac{K_{X}^{2}}{B^{2}}}
$$

where $B$ is as in Proposition 3.8 and $\mathcal{R}=\mathcal{R}_{\Omega_{X}}$.

Proof. The Bogomolov-Miyaoka-Yau inequality implies that part 1 of Theorem 3.11 cannot occur. We need to rule out the situation 2 as well.

The proof of Proposition 3.9 implies that $\left\{B_{x}\right\}_{x \in X}$ is a family of rational curves if $B^{2}=1$ and $\operatorname{deg}\left(\phi_{\Omega_{X}}\right)=2$. Since $X$ is a surface of general type, it follows that $\operatorname{deg}\left(\phi_{\Omega_{X}}\right) \geqslant 3$. This gives $K_{X}^{2}-\operatorname{deg}\left(c_{2}(X)\right) \geqslant \operatorname{deg}\left(\phi_{\Omega_{X}}\right) \cdot \operatorname{deg}\left(i m\left(\phi_{\Omega_{X}}\right)\right) \geqslant 9$. Combined with $\operatorname{deg}\left(c_{2}(X)\right)=\operatorname{deg}(\mathcal{R}) \operatorname{deg}(\pi)=$ $3 \operatorname{deg}(\pi) \geqslant 6$ we obtain $K_{X}^{2} \geqslant 15$. Since $\left(K_{X}^{2}+\operatorname{deg}\left(c_{2}(X)\right)\right)$ must be divisible by 12 and $\operatorname{deg}\left(c_{2}(X)\right)$ is divisible by 3 , we are left with the possibility $K_{X}^{2}=15$ and $\operatorname{deg}\left(c_{2}(X)\right)=9$. But then the holomorphic Euler characteristic

$$
\chi\left(\mathcal{O}_{X}\right)=\frac{K_{X}^{2}+\operatorname{deg}\left(c_{2}(X)\right)}{12}=2
$$

We claim that this is impossible. Indeed, if $X$ has no irrational pencil, then $p_{g}(X) \geqslant 2 q-3$, yielding $\chi\left(\mathcal{O}_{X}\right) \geqslant q-2 \geqslant 4$. So we may assume $p_{g}(X) \leqslant 2 q-4$, which, by Castelnuovo-de Franchis, implies that $X$ admits a fibration

$$
f: X \longrightarrow \Gamma
$$

over a smooth projective curve $\Gamma$ of genus $g_{\Gamma} \geqslant 2$. Let $F$ be the class of a smooth fibre of $f$ and let $g_{F}$ be its genus. Then it is well known that $K_{X}^{2} \geqslant 8\left(g_{\Gamma}-1\right)\left(g_{F}-1\right)$. Substituting $K_{X}^{2}=15$ we deduce $g_{\Gamma}=g_{F}=2$. But $q \leqslant g_{\Gamma}+g_{F} \leqslant 4$, contradicting the assumption that $q \geqslant 6$.

The last assertion is a combination of $B^{2} \geqslant 1$ and the inequality in Corollary 3.10.

\section{A 'Jacobian' of $\mathcal{E}$ and its invariants}

The definition of the cohomological invariant $\mathcal{R}_{\mathcal{E}}$ presented in $\S 1$ has the advantage of leading us rapidly to its definition $(\S 1)$ and its geometric interpretation $(\S 2)$. However, this definition is rather formal and may strike one as somewhat artificial. In fact, there is a more conceptual way to introduce $\mathcal{R}_{\mathcal{E}}$ : it emerges naturally from a variation of complex structure on $\mathcal{E}$. This is what we would like to discuss in this section.

Given $\mathcal{E}$, we have two obvious cup-products:

$$
\begin{aligned}
& \gamma_{1}: H^{1}\left(\mathcal{E}^{*}\right) \otimes H^{0}(\mathcal{E}) \longrightarrow H^{1}\left(\mathcal{O}_{X}\right), \\
& \gamma_{2}: S^{2} H^{1}\left(\mathcal{E}^{*}\right) \longrightarrow H^{2}\left(\mathcal{O}_{X}(-L)\right),
\end{aligned}
$$

from which we have built the sheaf $\mathcal{H}_{\mathcal{E}}$ (see (1.1)) and the morphism $\mathcal{R}_{2}$ (Remark 1.5) respectively. We will see shortly that the morphisms $\mathcal{R}_{k}$, for $k \geqslant 3$, emerge from a variation of the cup-product $\gamma_{2}$ as we deform the complex structure of $\mathcal{E}$ in a 'natural' way.

\subsection{A Jacobian, theta-divisor and the normal function of $\mathcal{E}$}

We begin by defining a space of 'natural' deformations of $\mathcal{E}$. Let $U_{\mathcal{E}}$ be the open subset of $\mathbf{P}=$ $\mathbf{P}\left(H^{0}(\mathcal{E})\right)$ introduced in $\S 1$. For $[e] \in U_{\mathcal{E}}$, consider the group of extensions $\operatorname{Ext}^{1}\left(\mathcal{I}_{Z_{e}}(L), \mathcal{O}_{X}\right)$. Since $\operatorname{dimExt}{ }^{1}\left(\mathcal{I}_{Z_{e}}(L), \mathcal{O}_{X}\right)=h^{1}\left(\mathcal{I}_{Z_{e}}\left(L+K_{X}\right)\right)=\delta_{\mathcal{E}}+1$, for all $[e] \in U_{\mathcal{E}}$, the spaces $\operatorname{Ext}^{1}\left(\mathcal{I}_{Z_{e}}(L), \mathcal{O}_{X}\right)$ fit together to form a vector bundle over $U_{\mathcal{E}}$. Denote this bundle $\mathbb{E x t}_{\mathbf{P}\left(H^{0}(\mathcal{E})\right)}^{1}$ (if no confusion is likely 


\section{REIDER}

we will abbreviate to $\left.\mathbb{E} \mathrm{xt}^{1}\right)$. To define it explicitly, consider the morphism

$$
\rho_{\mathcal{Z}_{\mathcal{E}}}: p_{1}^{*} \mathcal{O}_{X}\left(K_{X}+L\right) \longrightarrow \mathcal{O}_{\mathcal{Z}_{\mathcal{E}}} \otimes p_{1}^{*} \mathcal{O}_{X}\left(K_{X}+L\right)
$$

on $X \times U_{\mathcal{E}}$. Taking its direct image with respect to $p_{2}$ we obtain the morphism

$$
\rho_{\mathcal{E}}: H^{0}\left(\mathcal{O}_{X}\left(K_{X}+L\right)\right) \otimes \mathcal{O}_{U_{\mathcal{E}}} \longrightarrow p_{2 *}\left(\mathcal{O}_{\mathcal{Z}_{\mathcal{E}}} \otimes p_{1}^{*} \mathcal{O}_{X}\left(K_{X}+L\right)\right)
$$

whose cokernel is $\mathcal{R}^{1} p_{2 *}\left(\mathcal{I}_{\mathcal{Z}_{\mathcal{E}}} \otimes p_{1}^{*} \mathcal{O}_{X}\left(K_{X}+L\right)\right)$. We define $\mathbb{E x t}^{1}$ as

$$
\mathbb{E x t}^{1}=\left(\mathcal{R}^{1} p_{2 *}\left(\mathcal{I}_{\mathcal{Z}_{\mathcal{E}}} \otimes p_{1}^{*} \mathcal{O}_{X}\left(K_{X}+L\right)\right)\right)^{*} .
$$

Set $\mathbf{J}_{\mathbf{P}\left(H^{0}(\mathcal{E})\right)}=\mathbf{P}\left(\mathbb{E x t}{ }^{1}\right)$ (we will omit the subscript if there is no ambiguity). Let $q: \mathbf{J} \longrightarrow U_{\mathcal{E}}$ be the natural projection. Its fibre over $[e] \in U_{\mathcal{E}}$ is

$$
\mathbf{P}\left(H^{1}\left(\mathcal{I}_{Z_{e}}\left(K_{X}+L\right)\right)^{*}\right)=\mathbf{P}\left(\operatorname{Ext}^{1}\left(\mathcal{I}_{Z_{e}}(L), \mathcal{O}_{X}\right)\right),
$$

where the last equality is Serre duality on $X$. So $\mathbf{J}$ parametrizes a family of torsion-free sheaves of rank 2 with Chern classes $L$ and $c_{2}(\mathcal{E})$. Observe that the projection $q: \mathbf{J} \longrightarrow U_{\mathcal{E}}$ admits a section $s_{\mathcal{E}}: U_{\mathcal{E}} \longrightarrow \mathbf{J}$ which takes $[e] \in U_{\mathcal{E}}$ to the extension class $\left[\alpha_{e}\right] \in \mathbf{P}\left(\operatorname{Ext}^{1}\left(\mathcal{I}_{Z_{e}}(L), \mathcal{O}_{X}\right)\right)$ corresponding to the Koszul sequence of $e$,

$$
0 \longrightarrow \mathcal{O}_{X} \stackrel{e}{\longrightarrow} \mathcal{E} \stackrel{\wedge e}{\longrightarrow} \mathcal{I}_{Z_{e}}(L) \longrightarrow 0 .
$$

Definition 4.1. The section $s_{\mathcal{E}}: U_{\mathcal{E}} \longrightarrow \mathbf{J}$ is called the normal function of $\mathcal{E}$.

Let $\Sigma$ be the image of $s_{\mathcal{E}}$. We clearly can identify $U_{\mathcal{E}}$ with $\Sigma$. Deforming $\Sigma$ in $\mathbf{J}$ along the normal directions gives 'natural' deformations of $\mathcal{E}$ alluded to above.

We also observe that $\mathbf{J}$ carries a distinguished divisor $\Xi_{\mathbf{P}\left(H^{0}(\mathcal{E})\right)}$ such that

$$
\stackrel{\circ}{\mathbf{J}}=\mathbf{J}-\Xi
$$

parametrizes locally free extensions. In particular, the normal function of $\mathcal{E}$ takes its values in $\mathbf{J}$.

Remark 4.2. The divisor $\Xi$ can be defined explicitly as follows. An extension class

$$
\alpha \in \operatorname{Ext}^{1}\left(\mathcal{I}_{Z_{e}}(L), \mathcal{O}_{X}\right)
$$

defines a homomorphism

$$
H^{0}\left(\mathcal{O}_{Z_{e}}\right) \stackrel{\alpha}{\longrightarrow} \operatorname{Ext}^{2}\left(\mathcal{O}_{Z_{e}}(L), \mathcal{O}_{X}\right)=H^{0}\left(\mathcal{O}_{Z_{e}}\left(K_{X}+L\right)\right)^{*} .
$$

Furthermore, it is an isomorphism precisely when the rank-2 sheaf defined by $\alpha$ is locally free (see the proof of Claim 2.4 for details). Sheafifying this homomorphism over J yields

$$
\Upsilon: q^{*}\left(p_{2 *} \mathcal{O}_{\mathcal{Z}_{\mathcal{E}}}\right) \otimes \mathcal{O}_{\mathbf{J}}(-1) \longrightarrow q^{*}\left(p_{2 *}\left(\mathcal{O}_{\mathcal{Z}_{\mathcal{E}}} \otimes p_{1}^{*}\left(\mathcal{O}_{X}\left(K_{X}+L\right)\right)\right)^{*}\right),
$$

where $\mathcal{O}_{\mathbf{J}}(1)$ is such that $q_{*} \mathcal{O}_{\mathbf{J}}(1)=\mathbb{E x t}^{1^{*}}$. Thus $\Xi$ is the subscheme of $\mathbf{J}$, where $\Upsilon$ fails to be an isomorphism, i.e. $\Xi=(\operatorname{det} \Upsilon=0)$. In particular, fibres of $\Xi$ over $U_{\mathcal{E}}$ are hypersurfaces of degree $d$. More precisely, one can show that the support of $(\Xi)_{[e]}$, the fibre of $\Xi$ over $[e] \in U_{\mathcal{E}}$, is the union of hyperplanes $\bigcup_{p} H_{p}$ in $\mathbf{P}\left(\operatorname{Ext}^{1}\left(\mathcal{I}_{Z_{e}}(L), \mathcal{O}_{X}\right)\right)$, where $p$ runs through the support of $\kappa_{\mathcal{E}}\left(Z_{e}\right)$, so the divisor $\Xi$ determines the image of $\kappa_{\mathcal{E}}$ in Proposition 2.3.

The above considerations show that the pair $\left(\mathbf{J}_{\mathbf{P}\left(H^{0}(\mathcal{E})\right)}, \Xi_{\mathbf{P}\left(H^{0}(\mathcal{E})\right)}\right)$ is a rather natural analogue of the Jacobian of a curve with its theta-divisor. This is the reason for the following terminology.

Definition 4.3. $\mathbf{J}_{\mathbf{P}\left(H^{0}(\mathcal{E})\right)}$ (respectively, $\Xi_{\mathbf{P}\left(H^{0}(\mathcal{E})\right)}$ ) is called the Jacobian (respectively, the thetadivisor) of $\mathcal{E}$. 


\section{INVARIANTS OF VECTOR BUNDLES}

\subsection{The cohomological invariant of the Jacobian of $\mathcal{E}$}

On $X \times \mathbf{J}$ we have the universal extension defined as follows. Consider the diagram

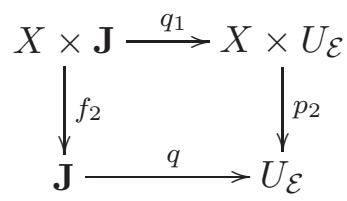

where $q_{1}=\operatorname{id}_{X} \times q$ and $f_{i}(i=1,2)$ is the projection of $X \times \mathbf{J}$ on the $i$ th factor. We fix $\mathcal{O}_{\mathbf{J}}(1)$ as in Remark 4.2 and consider the universal extension over $\mathbf{J}$,

$$
0 \longrightarrow f_{2}^{*} \mathcal{O}_{\mathbf{J}}(1) \longrightarrow \mathbf{E} \longrightarrow q_{1}^{*}\left(\mathcal{I}_{\mathcal{Z}_{\mathcal{E}}}\right) \otimes f_{1}^{*} \mathcal{O}_{X}(L) \longrightarrow 0
$$

given by the extension class in $\operatorname{Ext}^{1}\left(q_{1}^{*}\left(\mathcal{I}_{\mathcal{Z}_{\mathcal{E}}}\right) \otimes f_{1}^{*} \mathcal{O}_{X}(L), f_{2}^{*} \mathcal{O}_{\mathbf{J}}(1)\right)$ corresponding to $\operatorname{id}_{\mathbb{E x t}}{ }^{1}$ via the inclusion $\operatorname{End}\left(\mathbb{E x t}^{1}\right) \hookrightarrow \operatorname{Ext}^{1}\left(q_{1}^{*}\left(\mathcal{I}_{\mathcal{Z}_{\mathcal{E}}}\right) \otimes f_{1}^{*} \mathcal{O}_{X}(L), f_{2}^{*} \mathcal{O}_{\mathbf{J}}(1)\right)$. The extension (4.2) is a deformation of (1.2).

Next we describe a deformation of $c$ in (1.1) and $\mathcal{R}$ in (1.4). Tensor (4.2) with $f_{1}^{*} \mathcal{O}_{X}(-L)$ and combine it with $q_{1}^{*}$ of the defining sequence for $\mathcal{I}_{\mathcal{Z}_{\mathcal{E}}}$ to obtain the following diagram.

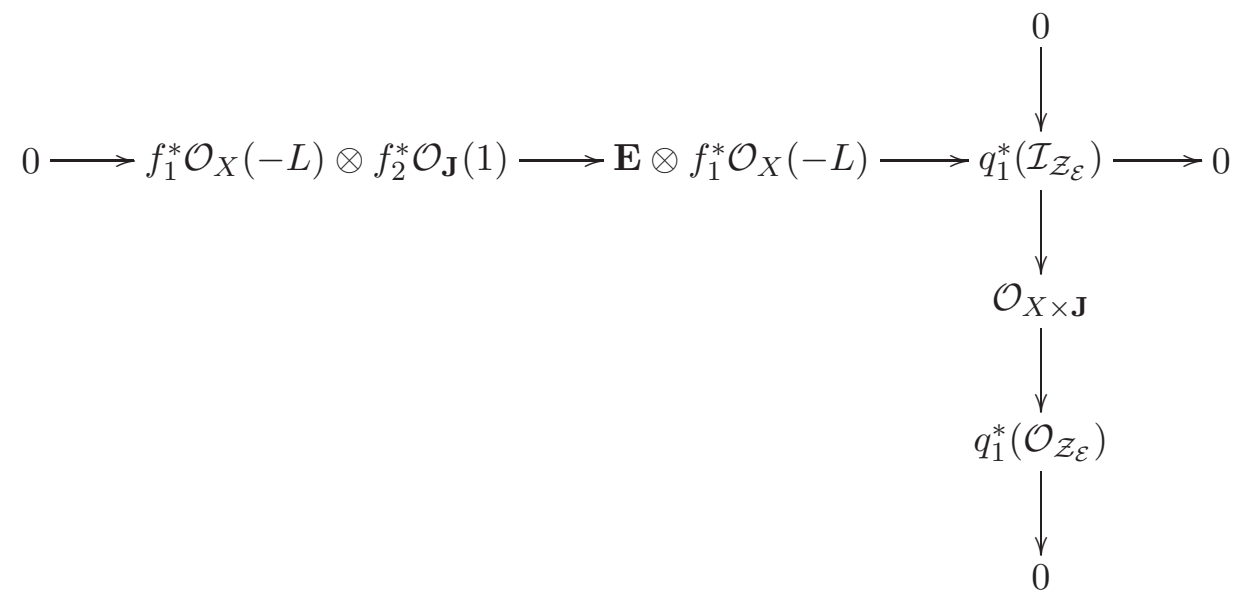

Taking the direct image with respect to $f_{2}$ we obtain the following diagram.

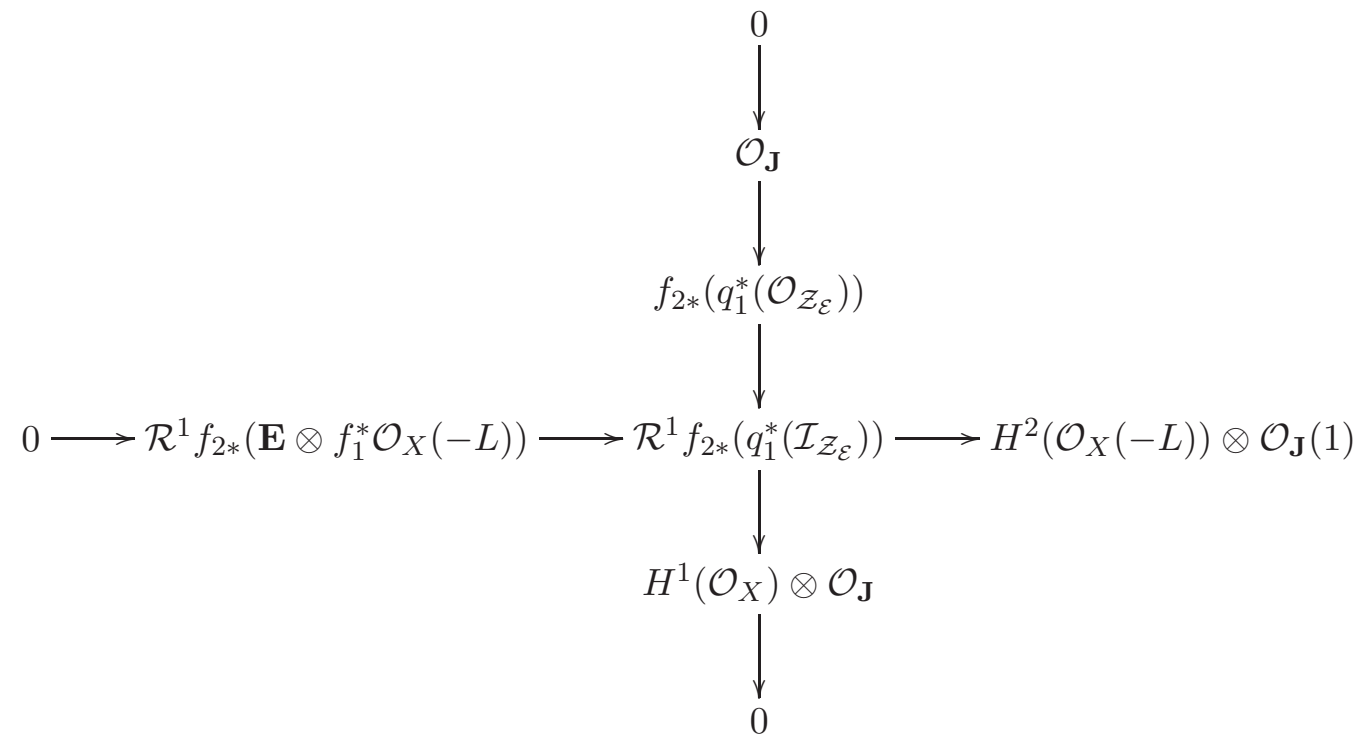

Since $p_{2}$ is a flat morphism we can use [Har77, Proposition 9.3, III] to rewrite the above diagram 


\section{REIDER}

as follows.

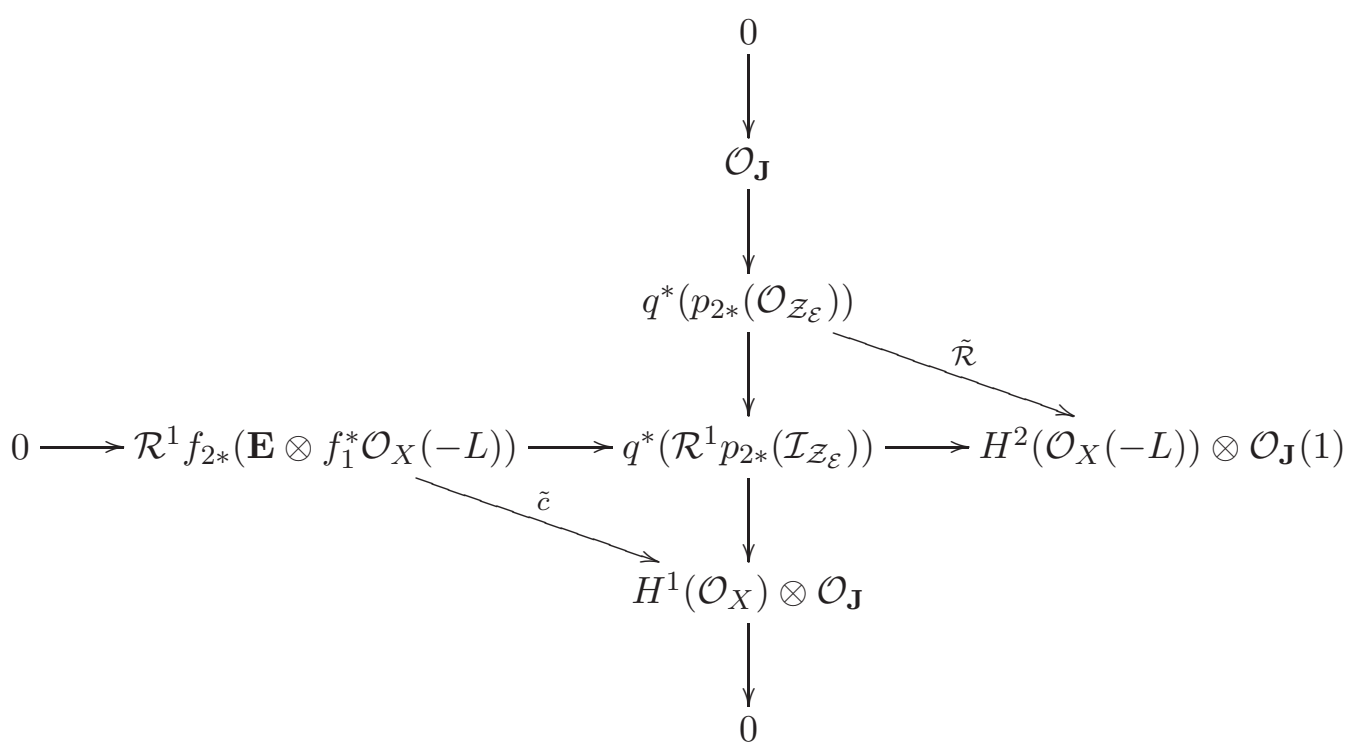

The morphism $\tilde{c}$ (respectively, $\tilde{\mathcal{R}}$ ) is a deformation of (1.1) (respectively, (1.4)).

Set $\mathcal{H}_{\mathbf{E}}=\operatorname{ker} \tilde{c}$ and $\tilde{\mathcal{H}}_{\mathbf{E}}=\operatorname{ker} \tilde{\mathcal{R}}$. Then we have

$$
0 \longrightarrow \mathcal{O}_{\mathbf{J}} \longrightarrow \tilde{\mathcal{H}}_{\mathrm{E}} \longrightarrow \mathcal{H}_{\mathrm{E}} \longrightarrow 0 \text {. }
$$

This is a deformation of (1.5).

Remark 4.4. The sequence (4.4) has a distinguished splitting. In fact, we have the trace morphism

$$
\operatorname{Tr}: p_{2 *} \mathcal{O}_{\mathcal{Z}_{\mathcal{E}}} \longrightarrow \mathcal{O}_{U_{\mathcal{E}}},
$$

which lifts to the morphism

$$
\tilde{\operatorname{Tr}}: q^{*}\left(p_{2 *} \mathcal{O}_{\mathcal{Z}_{\mathcal{E}}}\right) \longrightarrow \mathcal{O}_{\mathbf{J}} .
$$

Restricting it to $\tilde{\mathcal{H}}_{\mathbf{E}}$ and combining with (4.4) we obtain the following diagram.

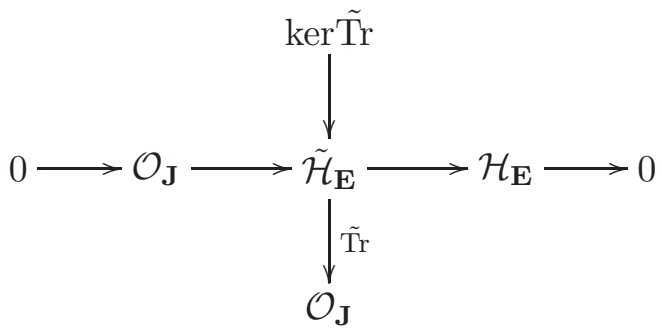

This induces an isomorphism

$$
\tilde{j}: \operatorname{ker} \tilde{\operatorname{Tr}} \longrightarrow \mathcal{H}_{\mathbf{E}}
$$

yielding a splitting

$$
\tilde{\mathcal{H}}_{\mathbf{E}}=\mathcal{O}_{\mathbf{J}} \oplus \mathcal{H}_{\mathbf{E}}
$$

Using the multiplicative structure of $q^{*}\left(p_{2 *} \mathcal{O}_{\mathcal{Z}_{\mathcal{E}}}\right)$ we have the morphisms

$$
\mathbf{m}_{k}: S^{k} \tilde{\mathcal{H}}_{\mathbf{E}} \longrightarrow q^{*}\left(p_{2 *} \mathcal{O}_{\mathcal{Z}_{\mathcal{E}}}\right)
$$

for every $k \geqslant 0$. Composing with $\tilde{\mathcal{R}}$ in (4.3) we obtain

$$
\tilde{\mathbf{R}}_{k}: S^{k} \tilde{\mathcal{H}}_{\mathbf{E}} \longrightarrow H^{2}\left(\mathcal{O}_{X}(-L)\right) \otimes \mathcal{O}_{\mathbf{J}}(1) \text {. }
$$




\section{INVARIANTS OF VECTOR BUNDLES}

The decomposition (4.5) implies that $S^{k} \mathcal{H}_{\mathbf{E}}$ is a direct summand of $S^{k} \tilde{\mathcal{H}}_{\mathbf{E}}$. Restricting $\tilde{\mathbf{R}}_{\mathbf{k}}$ to $S^{k} \mathcal{H}_{\mathbf{E}}$ we obtain the morphisms

$$
\mathbf{R}_{k}: S^{k} \mathcal{H}_{\mathbf{E}} \longrightarrow H^{2}\left(\mathcal{O}_{X}(-L)\right) \otimes \mathcal{O}_{\mathbf{J}}(1) .
$$

Definition 4.5. The sequence of morphisms $\mathbf{R}_{\mathbf{J}}:=\left\{\mathbf{R}_{k}\right\}_{k \in \mathbb{N}}$ is called the cohomological invariant of the Jacobian of $\mathcal{E}$.

Remark 4.6. We can view $\mathbf{R}_{k}$ as global sections of vector bundles $H^{2}\left(\mathcal{O}_{X}(-L)\right) \otimes\left(S^{k} \mathcal{H}_{\mathbf{E}}\right)^{*} \otimes \mathcal{O}_{\mathbf{J}}(1)$. This suggests that the cohomological invariant of $\mathbf{J}$ can be viewed as a non-Abelian analogue of the theta-functions.

Dualizing $\tilde{\mathbf{R}}_{k}$ and tensoring with $\mathcal{O}_{\mathbf{J}}(1)$ we obtain the morphism

$$
H^{0}(K+L) \otimes \mathcal{O}_{\mathbf{J}} \longrightarrow\left(S^{k} \tilde{\mathcal{H}}_{\mathbf{E}}\right)^{*} \otimes \mathcal{O}_{\mathbf{J}}(1) .
$$

Let $\tilde{\mathbf{F}}_{k}$ be its kernel. Then we obtain the following filtration:

$$
H^{0}(K+L) \otimes \mathcal{O}_{\mathbf{J}}=\tilde{\mathbf{F}}_{1} \supset \tilde{\mathbf{F}}_{2} \supset \cdots .
$$

Observe that the sheaf $\tilde{\mathcal{H}}_{\mathbf{E}}$ acts on the filtration $\tilde{\mathbf{F}}_{\bullet}$. Namely, we have the morphism

$$
\tilde{\mathcal{H}}_{\mathbf{E}} \otimes \tilde{\mathbf{F}}_{k} \longrightarrow \tilde{\mathbf{F}}_{k-1}
$$

for every $k \geqslant 2$. Combining with (4.4) yields

$$
a_{k}: \mathcal{H}_{\mathbf{E}} \longrightarrow \mathcal{H} \operatorname{Hom}\left(\tilde{\mathbf{F}}_{k} / \tilde{\mathbf{F}}_{k+1}, \tilde{\mathbf{F}}_{k-1} / \tilde{\mathbf{F}}_{k}\right)
$$

as well as the graded version of the cohomological invariant

$$
\operatorname{gr}_{k}\left(\mathbf{R}_{\mathbf{J}}\right): S^{k} \mathcal{H}_{\mathbf{E}} \longrightarrow\left(\tilde{\mathbf{F}}_{k-1} / \tilde{\mathbf{F}}_{k}\right)^{*} \otimes \mathcal{O}_{\mathbf{J}}(1)
$$

for every $k \geqslant 2$.

Observe that at this stage $\left(\mathbf{J}, \Xi_{\mathcal{E}}\right)$ and $\mathbf{R}_{\mathbf{J}}$ are the invariants of the incidence correspondence $p_{2}: \mathcal{Z}_{\mathcal{E}} \longrightarrow U_{\mathcal{E}}$ rather than of $\mathcal{E}$. One 'sees' $\mathcal{E}$ via its normal function, i.e. the section $s_{\mathcal{E}}: U_{\mathcal{E}} \longrightarrow \mathbf{J}^{\circ}$. In particular, one recovers the cohomological invariant of $\mathcal{E}$ defined in $\S 1$ as the pullback of $\mathbf{R}_{\mathbf{J}}$ under $s_{\mathcal{E}}$. This is obtained by computing the pullback of $\mathcal{H}_{\mathbf{E}}$ and $\mathcal{O}_{\mathbf{J}}(1)$ under $s_{\mathcal{E}}$.

Lemma 4.7. We have

1) $s_{\mathcal{E}}^{*} \mathcal{O}_{\mathbf{J}}(1)=\mathcal{O}_{U_{\mathcal{E}}}(-2)$,

2) $s_{\mathcal{E}}^{*} \mathcal{H}_{\mathbf{E}}=\mathcal{H}_{\mathcal{E}}(-1)$.

Proof. Identifying $U_{\mathcal{E}}$ with its image $\Sigma \subset \mathbf{J}$, the pullback becomes the restriction to $\Sigma$. So the first assertion follows from the fact that (4.2) restricted to $\Sigma$ is (1.2) tensored with $p_{1}^{*} \mathcal{O}_{X}(L)$. To see the second assertion, observe that the morphism $\Upsilon$ in (4.1) fits in the following commutative diagram.

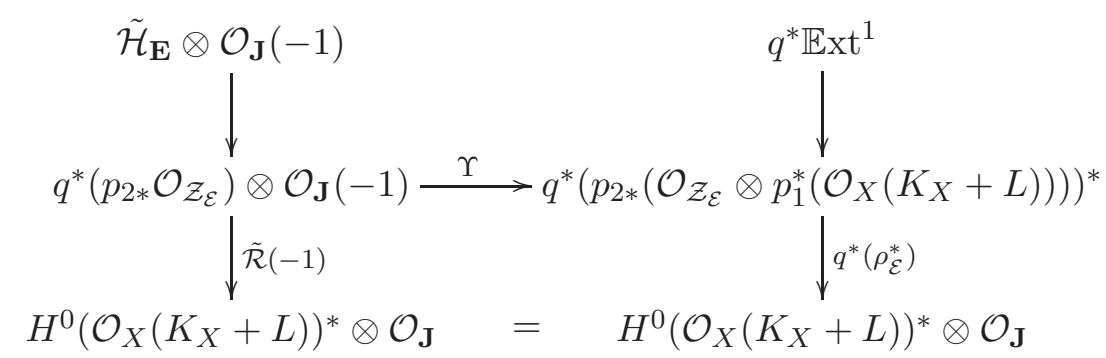

This yields a morphism

$$
\tilde{M}: \tilde{\mathcal{H}}_{\mathbf{E}} \otimes \mathcal{O}_{\mathbf{J}}(-1) \longrightarrow q^{*} \mathbb{E x t}^{1}
$$




\section{REIDER}

By Remark 4.2, $\Upsilon$ is an isomorphism over $\stackrel{\circ}{\mathbf{J}}$. This implies that $\tilde{M}$ is an isomorphism over $\stackrel{\circ}{\mathbf{J}}$ as well. Combining this with (4.4) we obtain

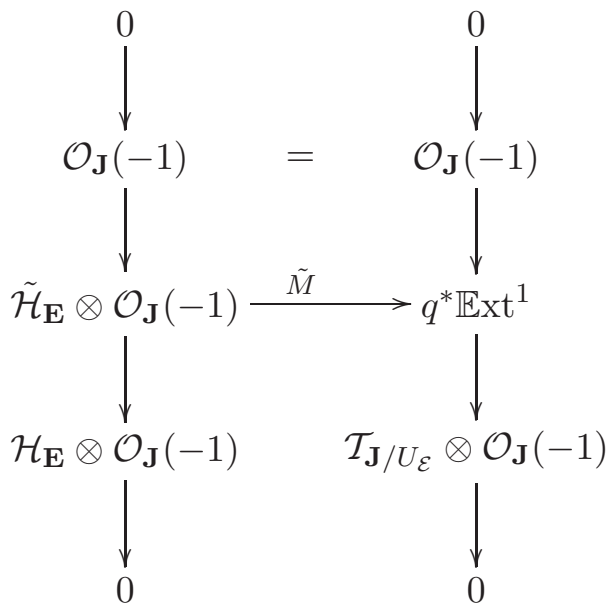

where $\mathcal{T}_{\mathbf{J} / U_{\mathcal{E}}}$ is the relative tangent bundle of $q: \mathbf{J} \longrightarrow U_{\mathcal{E}}$ and the column on the right is the relative Euler sequence tensored with $\mathcal{O}_{\mathbf{J}}(-1)$. Now the restriction of $q^{*} \mathbb{E x t}^{1}$ to $\Sigma$ can be computed from (1.2) tensored with $p_{1}^{*}\left(\mathcal{O}_{X}\left(L+K_{X}\right)\right)$. Taking the direct image with respect to $p_{2}$ we obtain the following diagram.

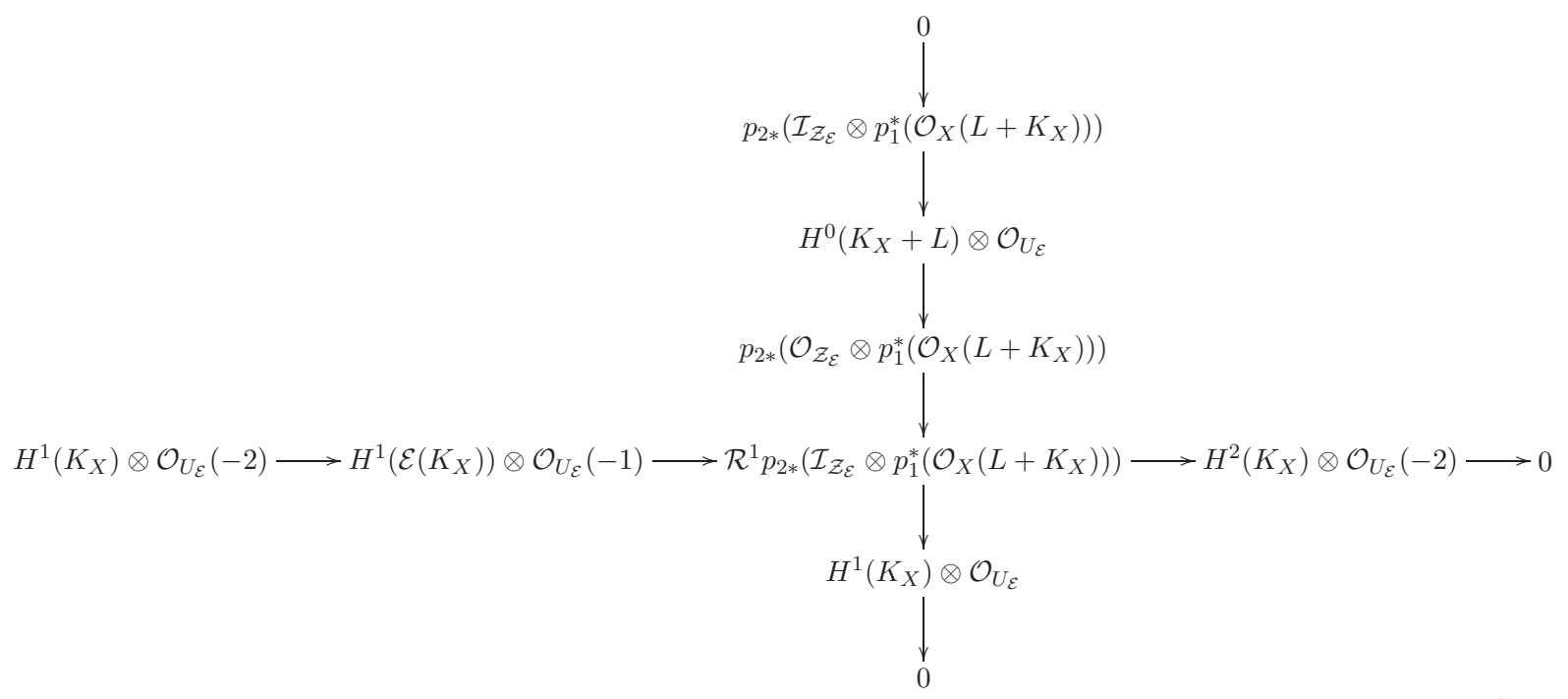

Dualizing this and comparing with (1.3) we deduce that

$$
\left(\mathcal{R}^{1} p_{2 *}\left(\mathcal{I}_{\mathcal{Z}_{\mathcal{E}}} \otimes p_{1}^{*}\left(\mathcal{O}_{X}\left(L+K_{X}\right)\right)\right)\right)^{*}=q^{*} \mathbb{E x t}^{1} \otimes \mathcal{O}_{\Sigma}=\tilde{\mathcal{H}}_{\mathcal{E}} \otimes \mathcal{O}_{\Sigma}(2) .
$$

This and (1.5) together with (4.15) yield

$$
\mathcal{H}_{\mathbf{E}} \otimes \mathcal{O}_{\mathbf{J}}(-1) \otimes \mathcal{O}_{\Sigma}=\mathcal{H}_{\mathcal{E}} \otimes \mathcal{O}_{\Sigma}(1)
$$

Combining with the first assertion of the lemma we obtain the second.

Remark 4.8. From (4.15) it follows that $\tilde{M}$ descends to the morphism

$$
M: \mathcal{H}_{\mathbf{E}} \longrightarrow \mathcal{T}_{\mathbf{J} / U_{\mathcal{E}}}
$$

which is an isomorphism over $\stackrel{\circ}{\mathbf{J}}$. So the restriction of the $\mathbf{R}_{k}$ to $\mathbf{J}_{\mathbf{J}}$ can be canonically identified as global sections of $H^{2}\left(\mathcal{O}_{X}(-L)\right) \otimes S^{k} \Omega_{\mathbf{J} / U_{\mathcal{E}}} \otimes \mathcal{O}_{\mathbf{J}_{\mathcal{E}}}(1)$, where $\Omega_{\mathbf{J} / U_{\mathcal{E}}}$ is the sheaf of relative differentials. 


\section{INVARIANTS OF VECTOR BUNDLES}

Thus, the cohomological invariant $\mathbf{R}_{\mathbf{J}}$ restricted to $\mathbf{J}$ can be viewed as a sequence of symmetric relative differentials on $\stackrel{\circ}{\mathcal{E}}_{\mathcal{E}}$ with values in $H^{2}\left(\mathcal{O}_{X}(-L)\right) \otimes \mathcal{O}_{\mathfrak{J}_{\mathcal{E}}}(1)$, or, equivalently, $\mathbf{R}_{k}$ can be viewed as a homogeneous polynomial of degree $k$ with values in $H^{2}\left(\mathcal{O}_{X}(-L)\right) \otimes \mathcal{O}_{\mathbf{J}_{\mathcal{E}}}(1)$ on the relative tangent bundle of $\mathbf{J}$, while $\mathbf{R}_{\mathbf{J}}$ can be thought of as a power series defined on the relative tangent bundle of $\stackrel{\circ}{\mathbf{J}}$ and taking its values in $H^{2}\left(\mathcal{O}_{X}(-L)\right) \otimes \mathcal{O}_{\mathfrak{J}_{\mathcal{E}}}(1)$. Restricting this series to the normal bundle of $\Sigma$ in $\stackrel{\circ}{\mathbf{J}}$ we recover the cohomological invariant $\mathcal{R}_{\mathcal{E}}$ of $\mathcal{E}$.

\subsection{The cohomological invariant and deformation of the cup-product $\gamma_{2}$}

Our 'natural' deformations of $\mathcal{E}$ produce deformations of the cup-product $\gamma_{2}$ which are behind the higher-order cohomological cup-products $\mathcal{R}_{k}$ entering in the definition of $\mathcal{R}_{\mathcal{E}}$. We will now explain this point.

On $\mathbf{J}^{\mathbf{J}}$ we have the relative cup-product

$$
\tilde{\gamma}_{2}: S^{2} \mathcal{R}^{1} f_{2 *}\left(\mathbf{E} \otimes f_{1}^{*} \mathcal{O}_{X}(-L)\right) \longrightarrow \mathcal{R}^{2} f_{2 *}\left(\wedge^{2}\left(\mathbf{E} \otimes f_{1}^{*} \mathcal{O}_{X}(-L)\right)\right)=H^{2}\left(\mathcal{O}_{X}(-L)\right) \otimes \mathcal{O}_{\mathbf{J}}(1),
$$

which is a deformation of $\gamma_{2}$. From (4.3) we obtain the following commutative diagram.

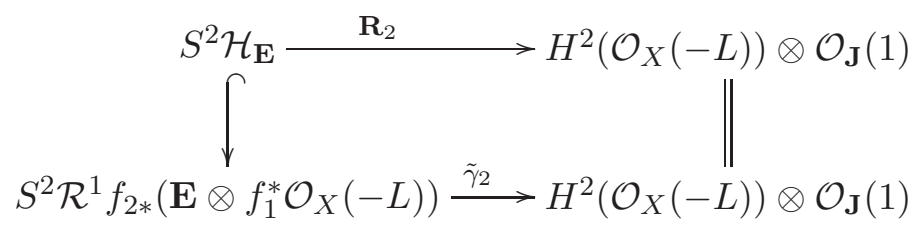

We are now in the position to differentiate the morphism $\mathbf{R}_{2}$ along the fibres of $q: \mathbf{J} \longrightarrow U_{\mathcal{E}}$. Set $\tilde{\mathbf{F}}_{1}=H^{0}\left(K_{X}+L\right) \otimes \mathcal{O}_{\mathbf{J}}$ and $\tilde{\mathbf{F}}_{2}=\operatorname{ker}\left(\mathbf{R}_{2}(-1)\right)^{*}$. If nonzero, this is a torsion-free sheaf on $\mathbf{J}$ and its rank denoted $\tilde{f}_{2}$ is well defined. Thus we obtain the following map.

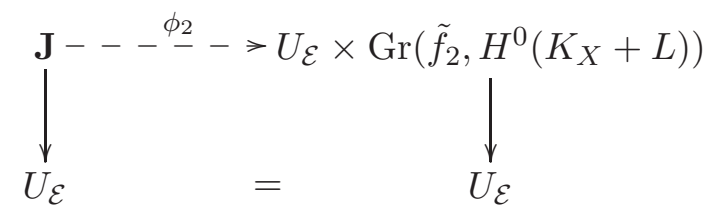

The relative differential of $\phi_{2}$ gives us a morphism

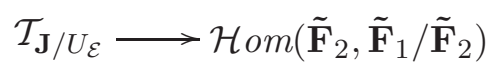

defined over an appropriate Zariski open subset of $\mathbf{J}$. This yields the morphism

$$
\tilde{\mathbf{F}}_{2} \longrightarrow\left(\mathcal{T}_{\mathbf{J} / U_{\mathcal{E}}}\right)^{*} \otimes\left(\tilde{\mathbf{F}}_{1} / \tilde{\mathbf{F}}_{2}\right) .
$$

Put $\tilde{\mathbf{F}}_{3}$ to be its kernel. Assuming it to be nonzero, we again obtain a torsion-free sheaf and denote its rank by $\tilde{f}_{3}$. As before this gives us a map into the Grassmannian, as follows.

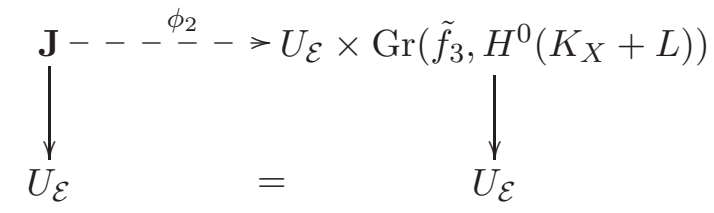

Taking its relative differential we obtain

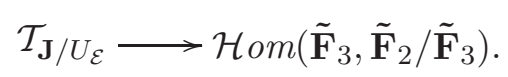

Continuing in this manner we obtain a decreasing filtration

$$
H^{0}\left(K_{X}+L\right) \otimes \mathcal{O}_{\mathbf{J}}=\tilde{\mathbf{F}}_{1} \supset \tilde{\mathbf{F}}_{2} \supset \cdots \supset \tilde{\mathbf{F}}_{k} \supset \cdots
$$




\section{REIDER}

together with morphisms

$$
\tilde{p}_{k}: \mathcal{T}_{\mathbf{J} / U_{\mathcal{E}}} \longrightarrow \mathcal{H} \operatorname{Hom}\left(\tilde{\mathbf{F}}_{k} / \tilde{\mathbf{F}}_{k+1}, \tilde{\mathbf{F}}_{k-1} / \tilde{\mathbf{F}}_{k}\right)
$$

for every $k \geqslant 2$. The main point of our discussion is that the filtration (4.18) is the same as the one in (4.9) (this is, of course, why we use the same notation) and the algebraically defined morphisms $a_{k}$ coincide with the diffeogeometric $\tilde{p}_{k}$ up to a constant multiple. More precisely, we have the following lemma.

Lemma 4.9. Over $\stackrel{\mathbf{J}}{\text { the diagram }}$

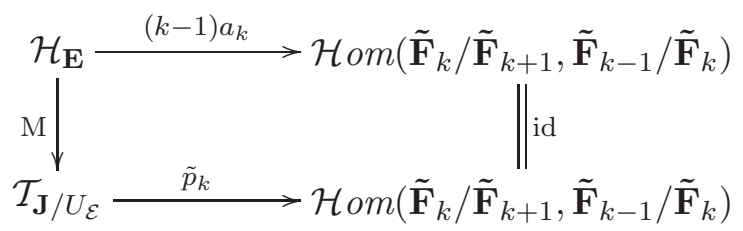

commutes, for every $k \geqslant 2$. (In other words, with the canonical identification $M$ in mind, we have the identity

for every $k \geqslant 2$.)

$$
\tilde{p}_{k}=(k-1) a_{k}
$$

A proof of Lemma 4.9 will be given in $\S 6$.

Iterating the morphisms $\tilde{p}_{k}$ we obtain

$$
\delta_{k-1}: S^{k-1} \mathcal{T}_{\mathbf{J} / U_{\mathcal{E}}} \longrightarrow \mathcal{H} \operatorname{Hom}\left(\tilde{\mathbf{F}}_{k} / \tilde{\mathbf{F}}_{k+1}, \tilde{\mathbf{F}}_{1} / \tilde{\mathbf{F}}_{2}\right)
$$

for every $k \geqslant 2$. Using the identification of $\mathcal{T}_{\mathbf{J} / U_{\mathcal{E}}}$ with $\mathcal{H}_{\mathbf{E}}$ over $\stackrel{\circ}{\mathbf{J}}$ (see Remark 4.8), we can rewrite $\mathbf{R}_{2}$ as

$$
S^{2} \mathcal{T}_{\mathbf{J} / U_{\mathcal{E}}} \longrightarrow H^{2}(-L) \otimes \mathcal{O}_{\mathbf{J}}(1)
$$

This morphism together with $\delta_{k-1}$ yields the morphisms

$$
\mathbf{R}_{2}^{(k-1)}: S^{k+1} \mathcal{T}_{\mathbf{J} / U_{\mathcal{E}}} \otimes \mathcal{O}_{\mathbf{J}} \longrightarrow\left(\tilde{\mathbf{F}}_{k} / \tilde{\mathbf{F}}_{k+1}\right)^{*} \otimes \mathcal{O}_{\mathbf{J}}(1)
$$

for every $k \geqslant 1$. This can be thought of as $(k-1)$ th-order derivative of $\mathbf{R}_{2}$ along the fibres of the projection $q: \stackrel{\circ}{\mathbf{J}} \longrightarrow U_{\mathcal{E}}$. Using (4.20) we deduce that $\operatorname{gr}_{k+1}\left(\mathbf{R}_{\mathbf{J}}\right)$ defined in (4.12) is related to $\mathbf{R}_{2}^{(k-1)}$ by the following 'Taylor coefficient' formula

$$
\operatorname{gr}_{k+1}\left(\mathbf{R}_{\mathbf{J}}\right)=\frac{1}{(k-1) !} \mathbf{R}_{2}^{(k-1)}
$$

for every $k \geqslant 1$. Thus, thinking of $\mathbf{R}_{2}$ as a 'function' on $\mathbf{J}$ (with values in $S^{2} \Omega_{\mathrm{J}} / U_{\mathcal{E}} \otimes \mathcal{O}_{\mathbf{J}}(1)$ ), we can regard $\mathbf{R}_{\mathbf{J}}$ as some kind of 'Taylor series' expansion of $\mathbf{R}_{2}$ along the fibres of the projection $q: \mathbf{J} \longrightarrow U_{\mathcal{E}}$. In particular, restricting the morphisms $\mathbf{R}_{2}^{(k-1)}$ to $\Sigma$ we obtain

$$
\mathcal{R}_{2}^{(k-1)}(\mathcal{E}):=\left.\mathbf{R}_{2}^{(k-1)}\right|_{\Sigma}: S^{k+1}\left(\mathcal{N}_{\Sigma / \mathbf{J}}\right) \longrightarrow\left(\tilde{\mathbf{F}}_{k} / \tilde{\mathbf{F}}_{k+1} \otimes \mathcal{O}_{\Sigma}\right)^{*} \otimes \mathcal{O}_{U_{\mathcal{E}}}(-2),
$$

where $\mathcal{N}_{\Sigma / \mathbf{J}}$ is the normal bundle of $\Sigma$ in $\mathbf{J}$. From the identification $\mathcal{N}_{\Sigma / \mathbf{J}}=\mathcal{T}_{\mathbf{J} / U_{\mathcal{E}}} \otimes \mathcal{O}_{\Sigma}$ we obtain

$$
\mathcal{R}_{2}^{(k-1)}(\mathcal{E}): S^{k+1}\left(\mathcal{T}_{\mathbf{J} / U_{\mathcal{E}}}\right) \otimes \mathcal{O}_{\Sigma} \longrightarrow\left(\tilde{\mathbf{F}}_{k} / \tilde{\mathbf{F}}_{k+1} \otimes \mathcal{O}_{\Sigma}\right)^{*} \otimes \mathcal{O}_{U_{\mathcal{E}}}(-2) .
$$

Using (4.17) and (4.20) we again have the 'Taylor coefficient' relation

$$
\operatorname{gr}_{k+1}\left(\mathcal{R}_{\mathcal{E}}\right)=\frac{1}{(k-1) !} \mathcal{R}_{2}^{(k-1)}(\mathcal{E})
$$




\section{INVARIANTS OF VECTOR BUNDLES}

where $\operatorname{gr}_{k+1}\left(\mathcal{R}_{\mathcal{E}}\right)$ is as in $(1.12)$. So the cohomological invariant $\mathcal{R}_{\mathcal{E}}$ can be viewed as the 'Taylor expansion' of the cup-product $\tilde{\gamma}_{2}$ around $\Sigma$ along its normal directions in $\mathbf{J}^{\circ}$.

\section{Two filtrations of the cohomological invariant}

The construction in $\S 1$ produces two filtrations. We have already encountered the decreasing filtration

$$
H^{0}\left(K_{X}+L\right) \otimes \mathcal{O}_{U_{\mathcal{E}}}=\tilde{\mathcal{F}}_{1} \supset \tilde{\mathcal{F}}_{2} \supset \cdots
$$

in (1.11). The other filtration is obtained by letting $\tilde{\mathcal{H}}_{-k}$ be the image of $m_{k}$ in (1.7). This yields a (decreasing) filtration

$$
0=\tilde{\mathcal{H}}_{1} \subset \tilde{\mathcal{H}}_{0} \subset \tilde{\mathcal{H}}_{-1} \subset \cdots \subset p_{2 *} \mathcal{O}_{\mathcal{Z}_{\mathcal{E}}}
$$

Remark 5.1. Observe that $\tilde{\mathcal{H}}_{0}=\mathcal{O}_{U_{\mathcal{E}}}$ and $\tilde{\mathcal{H}}_{-1}=\tilde{\mathcal{H}}_{\mathcal{E}}$.

We want to discuss some geometric properties of these filtrations. To begin with, observe that all nonzero sheaves involved in both filtrations are torsion-free. In particular, their ranks are well defined. From now on we will be working over the largest Zariski open subset of $\mathbf{P}$, where our filtrations are locally free. By abuse of notation we continue to denote this open subset by $U_{\mathcal{E}}$.

Definition 5.2. The function $P_{\mathcal{E}}(k)=\operatorname{rk}\left(\tilde{\mathcal{H}}_{-k}\right), k \in \mathbb{N}$, will be called the Hilbert function of $\mathcal{E}$.

We denote

$$
\Delta P_{\mathcal{E}}(k)=P_{\mathcal{E}}(k+1)-P_{\mathcal{E}}(k)=\operatorname{rk}\left(\tilde{\mathcal{H}}_{-k-1} / \tilde{\mathcal{H}}_{-k}\right)
$$

Our terminology obviously stems from the fact that $P_{\mathcal{E}}(k)$ is the Hilbert function of $\kappa_{\mathcal{E}}\left(Z_{e}\right)$ in $\mathbf{P}\left(\tilde{\mathcal{H}}_{\mathcal{E},[e]}^{*}\right)$ for every $[e] \in U_{\mathcal{E}}$.

The filtration (5.2) clearly controls the properties of the morphism $\kappa_{\mathcal{E}}$, e.g. the length of the filtration (5.2), denoted by $l_{\mathcal{E}}$, informs us that for every $[e] \in U_{\mathcal{E}}$ the subscheme $Z_{e}^{\prime}=\kappa_{\mathcal{E}}\left(Z_{e}\right)$ is $l_{\mathcal{E}}$-normal, i.e.

$$
S^{k} \tilde{\mathcal{H}}_{\mathcal{E},[e]} \longrightarrow H^{0}\left(\mathcal{O}_{Z_{e}^{\prime}}(k)\right)
$$

is surjective for all $k \geqslant l_{\mathcal{E}}$ and the homogeneous ideal $\bigoplus_{k \geqslant 0} H^{0}\left(\mathcal{I}_{Z_{e}^{\prime}}(k)\right)$ is generated in degrees $\leqslant\left(l_{\mathcal{E}}+1\right)$, while the fact that $\tilde{\mathcal{H}}_{-l_{\mathcal{E}}} \neq p_{2 *} \mathcal{O}_{\mathcal{Z}_{\mathcal{E}}}$ would imply that $\left.\kappa_{\mathcal{E}}\right|_{Z_{e}}$ fails to be an embedding. In particular, $P_{\mathcal{E}}\left(l_{\mathcal{E}}\right)$ is the length of $Z_{e}^{\prime}$ and $\operatorname{deg} \kappa_{\mathcal{E}}=d / P_{\mathcal{E}}\left(l_{\mathcal{E}}\right)>1$, whenever the degree of $\kappa_{\mathcal{E}}$ is defined.

The geometric meaning of the filtration (5.1) is perhaps less obvious. We will see in a moment that it not only contains information about the morphism $\kappa_{\mathcal{E}}$ but also tells us about the geometry of the $Z_{e}$ with respect to the linear system $\left|L+K_{X}\right|$.

The fact that the filtration (5.1) captures the properties of the morphism $\kappa_{\mathcal{E}}$ comes from its relationship with the filtration (5.2) given in the following lemma.

Lemma 5.3. For every $k \geqslant 1$ there is an isomorphism

$$
\tilde{\mathcal{H}}_{-k-1} / \tilde{\mathcal{H}}_{-k} \otimes \mathcal{O}_{U_{\mathcal{E}}}(2) \simeq\left(\tilde{\mathcal{F}}_{k} / \tilde{\mathcal{F}}_{k+1}\right)^{*}
$$

Proof. From the definition of the two filtrations it follows that $\mathcal{R}$ in (1.4) induces the following exact sequence:

$$
0 \longrightarrow \tilde{\mathcal{H}}_{-k} / \tilde{\mathcal{H}}_{-1} \otimes \mathcal{O}_{U_{\mathcal{E}}}(2) \longrightarrow H^{0}\left(K_{X}+L\right)^{*} \otimes \mathcal{O}_{U_{\mathcal{E}}} \longrightarrow\left(\tilde{\mathcal{F}}_{k}\right)^{*} \longrightarrow 0
$$




\section{REIDER}

These sequences fit together into the following commutative diagram.

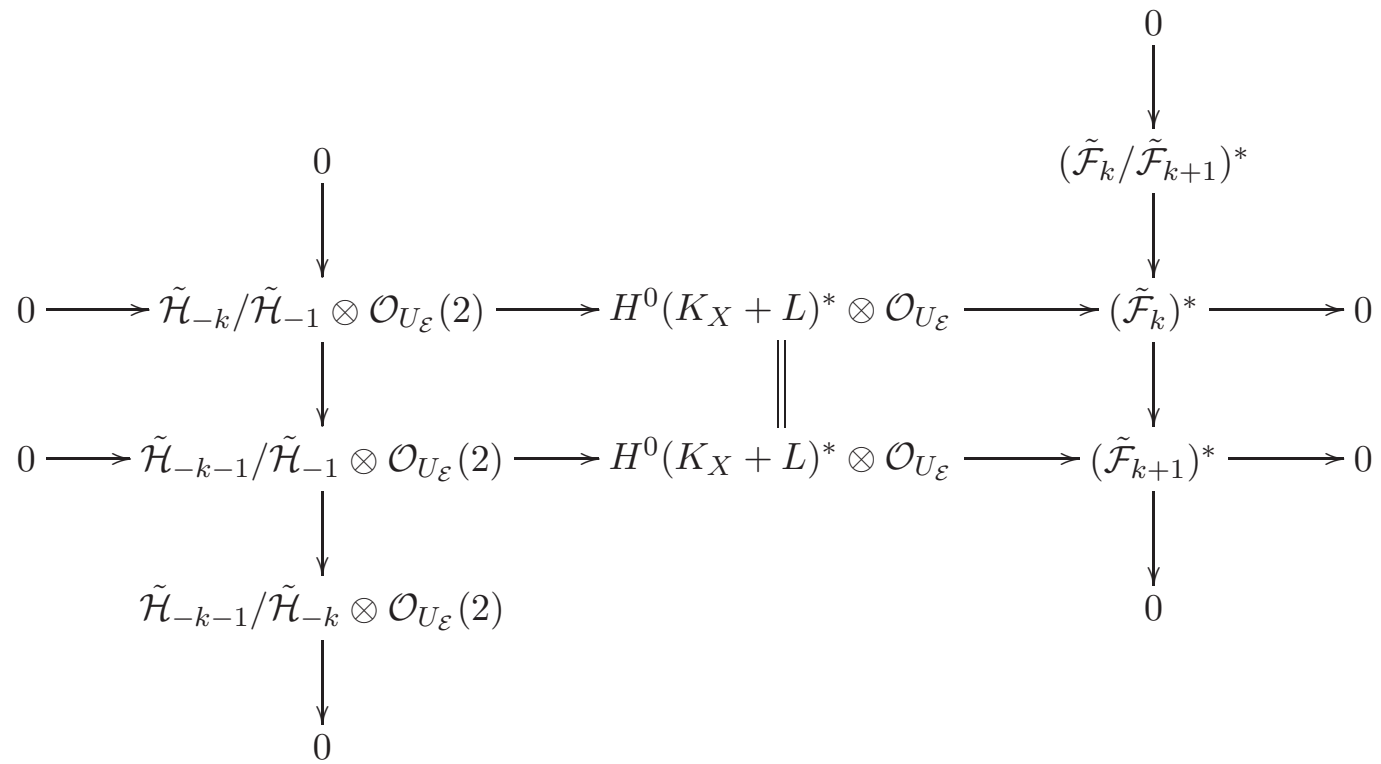

This implies the asserted isomorphism.

Proposition 5.4. For every $k \geqslant 1$ there is an inclusion

$$
p_{2 *}\left(\mathcal{I}_{\mathcal{Z}_{\mathcal{E}}} \otimes p_{1}^{*} \mathcal{O}_{X}\left(K_{X}+L\right)\right) \subset \tilde{\mathcal{F}}_{k} .
$$

Proof. From the definition of $\tilde{\mathcal{R}}_{k}$ it follows that $\tilde{\mathcal{F}}_{k} \supset \operatorname{ker}(\mathcal{R}(2))^{*}$ (see (1.4)). Comparing (4.16) with the dual of (1.3) tensored with $\mathcal{O}_{U_{\mathcal{E}}}(-2)$ we deduce that

$$
\operatorname{ker}(\mathcal{R}(2))^{*}=p_{2 *}\left(\mathcal{I}_{\mathcal{Z}_{\mathcal{E}}} \otimes p_{1}^{*} \mathcal{O}_{X}\left(K_{X}+L\right)\right) .
$$

Hence, the assertion of the proposition holds.

Set $\mathcal{F}_{k}=\tilde{\mathcal{F}}_{k} / p_{2 *}\left(\mathcal{I}_{\mathcal{Z}_{\mathcal{E}}} \otimes p_{1}^{*} \mathcal{O}_{X}\left(K_{X}+L\right)\right)$, for $k \geqslant 1$. This gives us the following filtration:

$$
p_{2 *}\left(\mathcal{O}_{\mathcal{Z}_{\mathcal{E}}} \otimes p_{1}^{*} \mathcal{O}_{X}\left(K_{X}+L\right)\right)=\mathcal{F}_{0} \supset \mathcal{F}_{1} \supset \cdots \supset \mathcal{F}_{l_{\mathcal{E}}}
$$

Corollary 5.5. We have $\operatorname{rk}\left(\mathcal{F}_{k}\right)=d-P_{\mathcal{E}}(k)$, for every $k \geqslant 1$. In particular, $\mathcal{F}_{l_{\mathcal{E}}}=0$ if and only if $\kappa_{\mathcal{E}}$ is an embedding.

Proof. From Lemma 5.3 it follows that

$$
\operatorname{rk}\left(\mathcal{F}_{i}\right)-\operatorname{rk}\left(\mathcal{F}_{i+1}\right)=\Delta P_{\mathcal{E}}(i)
$$

for every $i \geqslant 1$. Summing up these equalities for $1 \leqslant i \leqslant(k-1)$ we obtain

$$
P_{\mathcal{E}}(k)-P_{\mathcal{E}}(1)=\operatorname{rk}\left(\mathcal{F}_{1}\right)-\operatorname{rk}\left(\mathcal{F}_{k}\right) .
$$

This and $d=P_{\mathcal{E}}(1)+\operatorname{rk}\left(\mathcal{F}_{1}\right)$ imply the first assertion of the lemma. For the second assertion we take $k=l_{\mathcal{E}}$ to obtain $\operatorname{rk}\left(\mathcal{F}_{l_{\mathcal{E}}}\right)=d-P_{\mathcal{E}}\left(l_{\mathcal{E}}\right)$. This implies that $\operatorname{rk}\left(\mathcal{F}_{l_{\mathcal{E}}}\right)=0$ if and only if $d=P_{\mathcal{E}}\left(l_{\mathcal{E}}\right)$. But $P_{\mathcal{E}}\left(l_{\mathcal{E}}\right)$ is the length of $\kappa_{\mathcal{E}}\left(Z_{e}\right)$, for all $[e] \in U_{\mathcal{E}}$. Hence $\mathcal{F}_{l_{\mathcal{E}}}=0$ if and only if $\kappa_{\mathcal{E}}$ embeds $Z_{e}$ for every $[e] \in U_{\mathcal{E}}$, or, equivalently, $\kappa_{\mathcal{E}}$ is an embedding.

The above results show that the filtration $\tilde{\mathcal{F}}_{\bullet}$ in (5.1) contains as much information about the map $\kappa_{\mathcal{E}}$ as the filtration (5.2). We turn now to the properties of $\tilde{\mathcal{F}}_{\bullet}$ with respect to the linear system $\left|K_{X}+L\right|$. 


\section{INVARIANTS OF VECTOR BUNDLES}

The main point to observe is that the sheaf $p_{2 *}\left(\mathcal{O}_{\mathcal{Z}_{\mathcal{E}}}\right)$ acts on $p_{2 *}\left(\mathcal{O}_{\mathcal{Z}_{\mathcal{E}}} \otimes p_{1}^{*} \mathcal{O}_{X}\left(K_{X}+L\right)\right)$ via multiplication. From the definition of the filtration (5.4) it follows that the subsheaf $\tilde{\mathcal{H}}_{-1}$ acts not only on $p_{2 *}\left(\mathcal{O}_{\mathcal{Z}_{\mathcal{E}}} \otimes p_{1}^{*} \mathcal{O}_{X}\left(K_{X}+L\right)\right)$ but also on the filtration (5.4). More precisely, we have the morphism

$$
\tilde{\mathcal{H}}_{-1} \otimes \mathcal{F}_{k} \longrightarrow \mathcal{F}_{k-1}
$$

for every $k \geqslant 1$, i.e. $\tilde{\mathcal{H}}_{-1}$ acts on the filtration (5.4) by shifting the index by $(-1)$. The main geometric property of the morphisms (5.5) is that they define a linear subsystem of $\left|2\left(K_{X}+L\right)\right|$ vanishing on the $Z_{e}$. If, for example, $\mathcal{O}_{X}\left(K_{X}+L\right)$ is very ample, then what we actually claim is that the morphisms (5.5) give rise to the systems of quadrics passing through the image of the $Z_{e}$ under $\mathcal{O}_{X}\left(K_{X}+L\right)$.

To explain this point we put $W=\mathbf{P}\left(\mathcal{F}_{1}^{*}\right)$ and $\sigma: W \longrightarrow U_{\mathcal{E}}$ is the natural projection. Take $\mathcal{O}_{W}(1)$ such that $\sigma_{*} \mathcal{O}_{W}(1)=\mathcal{F}_{1}$. From now on we will assume that $\mathcal{O}_{X}\left(K_{X}+L\right)$ is base-point-free. The incidence cycle $p_{2}: \mathcal{Z}_{\mathcal{E}} \longrightarrow U_{\mathcal{E}}$ factors through $W$ to give us the following diagram.

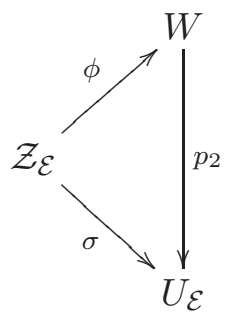

Let $\mathcal{V}$ be the image of $\phi$ and $\mathcal{I}_{\mathcal{V}}$ its sheaf of ideals. On $W$ we consider the relative Koszul complex

$$
0 \longrightarrow \mathcal{O}_{W} \longrightarrow \sigma^{*}\left(\sigma_{*} \mathcal{O}_{W}(1)\right)^{*} \otimes \mathcal{O}_{W}(1) \longrightarrow \sigma^{*}\left(\wedge^{2} \sigma_{*} \mathcal{O}_{W}(1)\right)^{*} \otimes \mathcal{O}_{W}(2) .
$$

The inclusion $\mathcal{F}_{k} \hookrightarrow \mathcal{F}_{1}$ gives the following complex

$$
0 \longrightarrow \mathcal{O}_{W} \longrightarrow \sigma^{*}\left(\mathcal{F}_{k}\right)^{*} \otimes \mathcal{O}_{W}(1) \longrightarrow \sigma^{*}\left(\wedge^{2} \mathcal{F}_{k}\right)^{*} \otimes \mathcal{O}_{W}(2)
$$

for every $k \geqslant 1$. This combined with the restriction morphism $\mathcal{O}_{W} \longrightarrow \mathcal{O}_{\mathcal{V}}$ yields

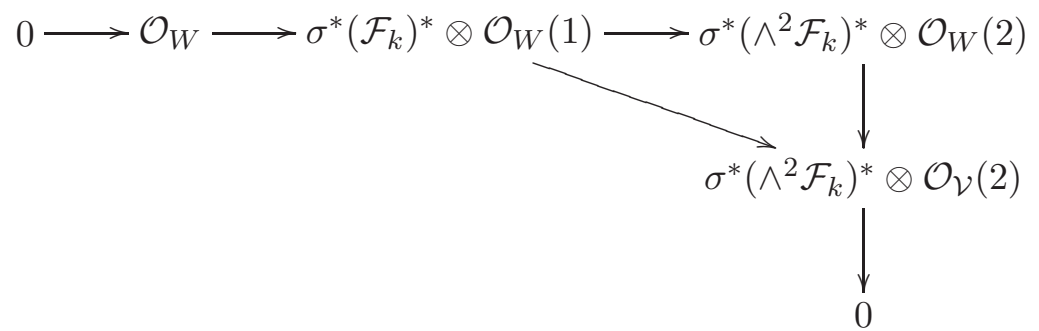

Taking the direct image with respect to $\sigma$ we obtain the following.

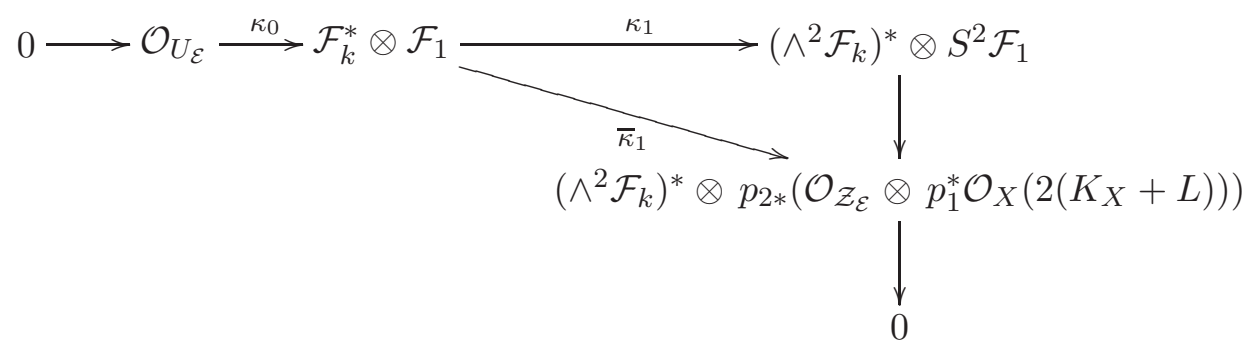

On the other hand we can rewrite (5.5) as morphisms

$$
\alpha_{k}: \tilde{\mathcal{H}}_{-1}=\tilde{\mathcal{H}}_{\mathcal{E}} \longrightarrow \mathcal{H} \text { om }\left(\mathcal{F}_{k}, \mathcal{F}_{k-1}\right)=\mathcal{F}_{k}^{*} \otimes \mathcal{F}_{k-1} .
$$

LEMma 5.6. We have $\bar{\kappa}_{1} \circ \alpha_{k}=0$, for every $k \geqslant 2$. 


\section{REIDER}

Proof. It is enough to show the assertion fibrewise. So we fix $[e] \in U_{\mathcal{E}}$ and observe that the morphism $\alpha_{k}$ at $[e]$ evaluated on $t \in \tilde{\mathcal{H}}_{\mathcal{E},[e]}$

$$
\alpha_{k}([e], t): \mathcal{F}_{k,[e]} \longrightarrow \mathcal{F}_{k-1,[e]}
$$

has the following property: $\alpha_{k}([e], t)(\psi)$ viewed as an element of $H^{0}\left(\mathcal{O}_{Z_{e}}\left(K_{X}+L\right)\right)$ is equal to $t \cdot \psi$, for every $\psi \in \mathcal{F}_{k,[e]}$. This implies the following relation in $H^{0}\left(\mathcal{O}_{Z_{e}}\left(2\left(K_{X}+L\right)\right)\right)$ :

$$
\psi^{\prime} \alpha_{k}([e], t)(\psi)-\psi \alpha_{k}([e], t)\left(\psi^{\prime}\right)=0,
$$

for every $\psi, \psi^{\prime} \in \mathcal{F}_{k,[e]}$.

Remark 5.7. From (5.6) we obtain the following complex:

$$
0 \longrightarrow \mathcal{O}_{U_{\mathcal{E}}} \stackrel{\kappa_{0}}{\longrightarrow} \mathcal{F}_{k}^{*} \otimes \mathcal{F}_{1} \stackrel{\bar{\kappa}_{1}}{\longrightarrow}\left(\wedge^{2} \mathcal{F}_{k}\right)^{*} \otimes p_{2 *}\left(\mathcal{O}_{\mathcal{Z}_{\mathcal{E}}} \otimes p_{1}^{*} \mathcal{O}_{X}\left(2\left(K_{X}+L\right)\right)\right)
$$

Let $\mathcal{K}_{k}^{1}$ be the cohomology sheaf of this complex at the middle term. Then the above lemma can be restated as follows: $\alpha_{k}$ induces the morphism

$$
\bar{\alpha}_{k}: \mathcal{H}_{\mathcal{E}} \otimes \mathcal{O}_{U_{\mathcal{E}}}(-1)=\tilde{\mathcal{H}}_{\mathcal{E}} / \tilde{\mathcal{H}}_{0} \longrightarrow \mathcal{K}_{k}^{1}
$$

for every $k \geqslant 2$. Composing $\bar{\alpha}_{k}$ with $\kappa_{1}$ we obtain

$$
\beta_{k}: \mathcal{H}_{\mathcal{E}} \otimes \mathcal{O}_{U_{\mathcal{E}}}(-1) \longrightarrow\left(\wedge^{2} \mathcal{F}_{k}\right)^{*} \otimes \sigma_{*}\left(\mathcal{I}_{\mathcal{V}}(2)\right)
$$

Thus, for every $[e] \in U_{\mathcal{E}}$ and every $t \in \mathcal{H}_{\mathcal{E}}([e])$ the linear map

$$
\beta_{k}([e], t): \wedge^{2} \mathcal{F}_{k,[e]} \longrightarrow H^{0}\left(\mathcal{I}_{V_{e}}(2)\right)
$$

yields a system of quadrics passing through $V_{e}$, the image of $Z_{e}$ under $\mathcal{O}_{X}\left(K_{X}+L\right)$.

We illustrate the above considerations in the following example.

Example 5.8. Let $\mathcal{E}$ be generated by global sections. Assume $d=\operatorname{deg}\left(c_{2}(\mathcal{E})\right) \geqslant 4$ and $\delta_{\mathcal{E}}=$ $\operatorname{deg} \mathcal{R}_{\mathcal{E}}=1$. Then the filtration (5.2) is a maximal ladder: $P_{\mathcal{E}}(k)=k+1$, for $0 \leqslant k \leqslant d-2$, and $P_{\mathcal{E}}(k)=d$, for $k \geqslant d-1$. From Corollary 5.5 it follows that the filtration

$$
\mathcal{F}_{1} \supset \mathcal{F}_{2} \supset \cdots \supset \mathcal{F}_{d-2} \supset \mathcal{F}_{d-1}=0
$$

is a maximal ladder as well. This implies that for $[e] \in U_{\mathcal{E}}$ the subscheme $Z_{e}$ is embedded in $\mathbf{P}\left(\mathcal{F}_{1,[e]}^{*}\right)=\mathbf{P}^{d-3}$ (here $\mathcal{F}_{k,[e]}^{*}$ is a fibre of $\mathcal{F}_{k}^{*}$ at $[e]$ ) and $Z_{e}$, viewed as a zero-dimensional subscheme of $\mathbf{P}^{d-3}$, is in general position. We will now count quadrics in $\mathbf{P}^{d-3}$ passing through $Z_{e}$.

Fix a nonzero element $t \in \mathcal{H}_{\mathcal{E}}([e])$, where $\mathcal{H}_{\mathcal{E}}([e])$ denotes the fibre of $\mathcal{H}_{\mathcal{E}}$ at $[e]$. By Remark 5.7 we have

$$
\beta_{k}([e], t): \wedge^{2} \mathcal{F}_{2,[e]} \longrightarrow H^{0}\left(\mathcal{I}_{Z_{e}}(2)\right) .
$$

One can show that this map is injective (see also below). Hence, $Z_{e}$ lies at least on $\left(\begin{array}{c}d-3 \\ 2\end{array}\right)$ quadrics. By a Lemma of Castelnuovo [GH78a], $Z_{e}$ lies on a rational normal curve cut out by these $\left(\begin{array}{c}d-3 \\ 2\end{array}\right)$ quadrics. In fact, our construction allows us to write down the quadratic equations defining this rational normal curve: Choose a lifting $\tilde{t} \in \tilde{\mathcal{H}}_{\mathcal{E},[e]}$ of $t$ and fix a basis vector $\phi_{d-2} \in \mathcal{F}_{d-2,[e]}$; using the action of $\tilde{t}$ on the filtration $\mathcal{F}_{\bullet,[e]}($ see $(5.5))$ we obtain a basis $\left\{\phi_{k}\right\}$ of $\mathcal{F}_{1,[e]}$ subject to the condition

$$
\phi_{k}=\alpha_{k+1}([e], \tilde{t})\left(\phi_{k+1}\right)
$$

for $k=1, \ldots, d-3$. From Remark 5.7 it follows that the image of $\beta_{k}([e], t)$ is spanned by the minors of the following matrix

$$
\left(\begin{array}{c}
\phi_{d-2} \ldots \phi_{2} \\
\phi_{d-3} \ldots \phi_{1}
\end{array}\right)
$$

It is well known that this defines a rational normal curve. 


\section{INVARIANTS OF VECTOR BUNDLES}

The appearance of the rational curves passing through the $Z_{e}$ is also a part of our construction: these are the fibres of $\mathbf{J}$, our space of 'natural' deformations of $\mathcal{E}$ (see $\S 3$ ), which in the case at hand is a $\mathbf{P}^{1}$-bundle over $U_{\mathcal{E}}$. Thus the Castelnuovo rational curve passing through $Z_{e}$ acquires a 'modular' meaning: it parametrizes a family of torsion-free sheaves of rank 2 on $X$ whose Chern classes are $\left(L,\left[Z_{e}\right]\right)$. Furthermore, the points $Z_{e}$ (viewed as a subset of the Castelnuovo curve) correspond to the members of the family which are not locally free.

\section{Proof of Lemma 4.9}

Let $[e] \in U_{\mathcal{E}}$ and put $Z=Z_{e}=(e=0)$. Consider the restriction of $\tilde{M}$ (see (4.14)) to the fibre of $q: \mathbf{J} \longrightarrow U_{\mathcal{E}}$ over $[e]:$

$$
\tilde{M}_{e}: \tilde{\mathcal{H}}_{\mathbf{E}} \otimes \mathcal{O}_{\mathbf{J}_{Z}}(-1) \longrightarrow \operatorname{Ext}_{Z}^{1} \otimes \mathcal{O}_{\mathbf{J}_{Z}}
$$

where $\stackrel{\circ}{\mathbf{J}}_{Z}=\stackrel{\circ}{\mathbf{J}} \cap q^{-1}([e])$ and $\operatorname{Ext}_{Z}^{1}=\operatorname{Ext}^{1}\left(\mathcal{I}_{Z_{e}}(L), \mathcal{O}_{X}\right)$. The morphism $\tilde{M}_{e}$ at $[\alpha] \in \stackrel{\circ}{Z}_{Z}$ is given by the cup-product with $\alpha$

$$
H^{0}\left(\mathcal{O}_{Z}\right) \stackrel{\alpha}{\longrightarrow} H^{0}\left(\mathcal{O}_{Z}\left(K_{X}+L\right)\right)^{*}
$$

(see the proof of Claim 2.4) and the fibre of $\tilde{\mathcal{H}}_{\mathbf{E}} \otimes \mathcal{O}_{\mathbf{J}_{Z}}$ at $[\alpha]$ can be described by

$$
\tilde{\mathcal{H}}_{\mathbf{E}}([\alpha])=\left\{f \in H^{0}\left(\mathcal{O}_{Z}\right) \mid\langle\alpha f, \phi\rangle=0, \forall \phi \in i m\left(\rho_{Z}\right)\right\}
$$

where $\rho_{Z}: H^{0}\left(\mathcal{O}_{X}\left(K_{X}+L\right)\right) \longrightarrow H^{0}\left(\mathcal{O}_{Z}\left(K_{X}+L\right)\right)$ and $\langle\cdot, \cdot\rangle$ denotes the obvious pairing between vectors and covectors.

We will need to know how $\tilde{\mathcal{H}}_{\mathbf{E}}([\alpha])$ changes when $[\alpha]$ moves in $\stackrel{\circ}{Z}_{Z}$. For this we use the isomorphism

$$
\operatorname{Ext}^{2}\left(\mathcal{O}_{Z}, \mathcal{O}_{X}(-L)\right)=H^{0}\left(\omega_{Z} \otimes \mathcal{O}_{X}\left(-K_{X}-L\right)\right)
$$

where $\omega_{Z}$ is the dualizing sheaf of $Z$. Then the elements $[\alpha] \in \mathbf{J}_{Z}$ correspond to the elements of $\operatorname{Ext}_{Z}^{1} \subset \operatorname{Ext}^{2}\left(\mathcal{O}_{Z}, \mathcal{O}_{X}(-L)\right)$ which are nowhere vanishing as sections of $\omega_{Z} \otimes \mathcal{O}_{X}\left(-K_{X}-L\right)$. This implies that for any $[\alpha],[\beta] \in \stackrel{\circ}{\mathbf{J}}_{Z}$ we have an isomorphism

$$
t: \tilde{\mathcal{H}}_{\mathbf{E}}([\alpha]) \longrightarrow \tilde{\mathcal{H}}_{\mathbf{E}}([\beta])
$$

given by the multiplication with $t=\alpha / \beta \in H^{0}\left(\mathcal{O}_{Z}\right)$. Thus we obtain

$$
\tilde{\mathcal{H}}_{\mathbf{E}}([\beta])=\frac{\alpha}{\beta} \tilde{\mathcal{H}}_{\mathbf{E}}([\alpha]) \text {. }
$$

Fix $[\alpha] \in \stackrel{\circ}{Z}_{Z}$ and consider an arc $\alpha(\varepsilon)$ passing through $[\alpha](=\alpha(0))$ with a tangent $\tau$ at $[\alpha]$. Lifting to $\operatorname{Ext}_{Z}^{1}$ we may consider the $\operatorname{arc} \tilde{\alpha}(\varepsilon)=\alpha+\varepsilon \beta$, where $\beta \in \operatorname{Ext}_{Z}^{1}$ is such that $\tau \equiv \beta$ $(\bmod \alpha)$ (we use the canonical identification of the tangent space of $\mathbf{J}_{Z}^{\circ}$ at $[\alpha]$ with $\operatorname{Ext}_{Z}^{1} /\langle\alpha\rangle$ ). Put $f(\varepsilon)=\alpha / \tilde{\alpha}(\varepsilon)$. From (6.1) we deduce that

$$
\tilde{\mathcal{H}}_{\mathbf{E}}(\alpha(\varepsilon))=f(\varepsilon) \cdot \tilde{\mathcal{H}}_{\mathbf{E}}([\alpha])=\frac{1}{1+\varepsilon t} \cdot \tilde{\mathcal{H}}_{\mathbf{E}}([\alpha]),
$$

where $t=\beta / \alpha \in H^{0}\left(\mathcal{O}_{Z}\right)$.

Let $\tilde{\mathbf{F}}_{k}([\alpha])$ be the fibre of $\tilde{\mathbf{F}}_{k} \otimes \mathcal{O}_{\tilde{\mathbf{J}}_{Z}}$ at $[\alpha]$. Take $\phi$ in $\tilde{\mathbf{F}}_{k}([\alpha])$ and let

$$
\phi(\varepsilon)=\phi+\varepsilon \psi_{1}+\varepsilon^{2} \psi_{2}+\cdots
$$

be a local section of $\tilde{\mathbf{F}}_{k} \otimes \mathcal{O}_{\mathbf{J}_{Z}}$ over $\alpha(\varepsilon)$. By definition, $\tilde{p}_{k}([e],[\alpha])(\tau)$ takes $\phi$ to the equivalence class of $\psi_{1}$ in $\tilde{\mathbf{F}}_{k-1}([\alpha]) / \tilde{\mathbf{F}}_{k}([\alpha])$. We will express it as follows:

$$
\tilde{p}_{k}([e],[\alpha])(\tau)(\phi) \equiv \psi_{1} \quad\left(\bmod \tilde{\mathbf{F}}_{k}([\alpha])\right) .
$$




\section{INVARIANTS OF VECTOR BUNDLES}

Since $\phi(\varepsilon) \in \tilde{\mathbf{F}}_{k}(\alpha(\varepsilon))$ we have

$$
\langle\tilde{\alpha}(\varepsilon) \cdot x(\varepsilon), \phi(\varepsilon)\rangle=0
$$

for every $x(\varepsilon) \in S^{k}\left(\tilde{\mathcal{H}}_{\mathbf{E}}(\alpha(\varepsilon))\right)$. By $(6.2)$ we can take $x(\varepsilon)=(f(\varepsilon))^{k} \cdot x$, where $x \in S^{k} \tilde{\mathcal{H}}_{\mathbf{E}}([\alpha])$. Substituting this in (6.4) we obtain

$$
\left\langle\alpha \cdot(f(\varepsilon))^{k-1} \cdot x, \phi(\varepsilon)\right\rangle=0 .
$$

Taking the expansion of $f(\varepsilon)$ in powers of $\varepsilon$ we have

$$
0=\left\langle\alpha \cdot\left(1-\varepsilon(k-1) t+o\left(\varepsilon^{2}\right)\right) \cdot x, \phi+\varepsilon \psi_{1}+o\left(\varepsilon^{2}\right)\right\rangle .
$$

Collecting the linear terms yields

$$
\langle\alpha \cdot(k-1) t \cdot x, \phi\rangle=\left\langle\alpha \cdot x, \psi_{1}\right\rangle
$$

for all $x \in S^{k} \tilde{\mathcal{H}}_{\mathbf{E}}([\alpha])$. This implies

$$
\left(\psi_{1}-(k-1) t \phi\right) \in \tilde{\mathbf{F}}_{k}([\alpha]) .
$$

Combining with (6.3) we obtain

$$
\tilde{p}_{k}([e],[\alpha])(\tau)(\phi) \equiv(k-1) t \phi \quad\left(\bmod \tilde{\mathbf{F}}_{k}([\alpha])\right) .
$$

By definition $M($ see $(4.17))$ takes the equivalence class $[t] \in \tilde{\mathcal{H}}_{\mathbf{E}}([\alpha]) / H^{0}\left(\mathcal{O}_{X}\right)=\mathcal{H}_{\mathbf{E}}([\alpha])$ to the tangent vector $\tau$ while the definition of $a_{k}$ (see (4.11)) implies

$$
a_{k}([e],[\alpha])([t])(\phi) \equiv t \phi \quad\left(\bmod \tilde{\mathbf{F}}_{k}([\alpha])\right) .
$$

This combined with (6.6) yields the assertion of the lemma.

\section{REFERENCES}

GH78a P. Griffiths and J. Harris, Principles of algebraic geometry (Wiley, New York, 1978).

GH78b P. Griffiths and J. Harris, Residues and zero-cycles on algebraic varieties, Ann. of Math. (2) 108 (1978), 461-505.

Har77 R. Hartshorne, Algebraic geometry (Springer, Berlin, 1977).

OSS80 C. Okonek, M. Schneider and H. Spindler, Vector bundles on complex projective space, Progress in Mathematics, vol. 3 (Birkhäuser, Boston, 1980).

Rei77 M. Reid, Bogomolov's theorem $c_{1}^{2} \leqslant 4 c_{2}$, in Proceedings of the international symposium on algebraic geometry (Kyoto Univ., Kyoto, 1977) (Kinokuniya Book Store, Tokyo, 1978), 623-642.

Tyu87 A. N. Tyurin, Cycles, curves and vector bundles on an algebraic surface, Duke Math. J. 54 (1987), $1-26$.

Igor Reider reider@univ-angers.fr

Université d'Angers, Département de Mathématiques, 2 Boulevard Lavoisier, 49045 Angers Cedex 01, France 